GEOLOGICAL SURVEY CIRCULAR 306

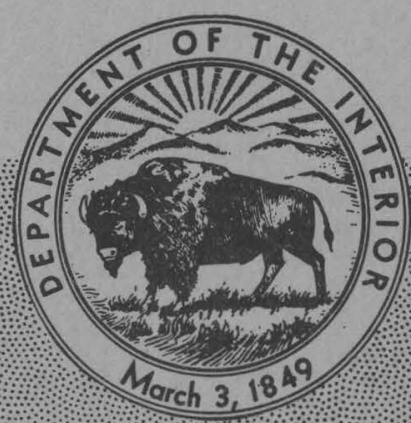

STRATIGRAPHIC SECTIONS OF THE PHOSPHORIA FORMATION IN

UTAH, 1949-51 

UNITED STATES DEPARTMENT OF THE INTERIOR

Douglas McKay, Secretary

GE OLOGICAL SURVEY

W. E. Wrather, Director

GEOLOGICAL SURVEY CIRCULAR 306

\section{STRATIGRAPHIC SECTIONS OF THE PHOSPHORIA FORMATION IN UTAH, 1949-51}

By T. M. Cheney, R. A. Smart, R. G. Waring, and M. A. Warner

This report concerns work done on

behalf of the U. S. Atomic Energy

Commission and is published with

the permission of the Commission. 
. 


\title{
STRATIGRAPHIC SECTIONS OF THE PHOSPHORIA FORMATION IN UTAH, 1949-51
}

\author{
By T. M. Cheney, R. A. Smart, R. G. Waring, and M. A. Warner
}

CONTENTS

Lot no. Page

Lot no. Page

Introduction. . . . . . . . . . . . . Acknowledgments............... Stratigraphy of the Phosphoria. and Park City formations

in $\mathrm{Utah} . . . \ldots \ldots \ldots \ldots \ldots \ldots \ldots$

Stratigraphic sections........... Selected bibliography ........... Tables of stratigraphic sections Laketown Canyon........... 1290
1

1

4

4

4

5 Tables of stratigraphic
sections-Continued North Crawford. . . . . . . . . . $1292 \quad 7$ Devils Slide.............. $1345 \quad 11$ Franson Canyon .............. $1347 \quad 18$ Horseshoe Canyon ........... $1339 \quad 22$ Fort Douglas............... $1350 \quad 25$ Sols Canyon............... $1348 \quad 32$ Strawberry Valley ........... $1371 \quad 35$

Little Diamond Creek ........... 1376

\section{ILLUSTRATIONS}

Figure 1. Outcrops of the Phosphoria and Park City formations in Utah and localities sampled............

2. Generalized section of the Phosphoria formation at Brazer Canyon, Utah, lot no. 1203 .......

\section{INTRODUCTION}

As part of a comprehensive investigation of the phosphate deposits of the western field begun in 1947, the U. S. Geological Survey has measured and sampled the full thickness of the Permian Phosphoria formation and its partial correlative, the Park City formation, at many localities in Utah and other western states. Although these data will not be fully analyzed for several years, segments of the data, accompanied by little or no interpretation, are published as preliminary reports as they are assembled. This report, which contains abstracts of the sections measured in northeastern Utah (fig. 1) in 1949, 1950, and 1951, is one of this series and the second report of data gathered in Utah. The field and laboratory procedures adopted in these investigations are described rather fully in a previous report (McKelvey and others, 1953a).

A large number of people have taken part in this investigation. The program of which this work is a part wa.s organized by $V$. E. McKelvey and the field program was supervised by R. W. Swanson. R. P. Sheldon assisted in the location and selection of sample localities and F. J. Anderson, McKelvey, Sheldon, and $H$. W. Peirce participated in the description of strata. and the collection of samples referred to in this report. $T . K$. Rigby assisted in the preparation of trenches and the crushing and splitting of samples in the field. The laboratory preparation of samples for chemical analysis was done in Denver, Colo., under the direction of W. P. Huleatt.
The $\mathrm{P}_{2} \mathrm{O}_{5}$ and acid-insoluble analyses were made in part in the Trace Elements Section laboratory of the Survey in Denver, Colo., under the direction of L. F. Rader, by chemists M. Appling, J. Dufour, N. Jammer, H. Miskowiec, N. Morris, and M. Stevens and in part for the Survey by the U. S. Bureau of Mines at the Northwest Electrodevelopment Laboratory, Albany; Oreg., under the direction of S. M. Shelton and $\mathrm{M}$. L. Wright. The $\mathrm{Al}_{2} \mathrm{O}_{3}, \mathrm{Fe}_{2} \mathrm{O}_{3}$, and loss-onignition analyses were made in the Trace Elements Section laboratory, in Washington, D. C., under the direction of J. C. Rabbitt, by H. Alberty, T. Farley, C. Hoy, F. Grimaldi, and M. Landers.

Compilation of the data has been largely by $K$. S. Bergman under the supervision of R. W. Swanson. Organization of the tabular data has been by Anita Wise.

\section{ACKNOW LEDGMENTS}

Special thanks are due J. Steele Williams and A. A. Baker who have given much advice and many suggestions in the field. The cost of these investigations has been borne partly by the Division of Raw Materials of the Atomic Energy Commission.

It is a pleasure to acknowledge the fine cooperation extended to the field parties by the local residents and property owners who furnished information and services and gave access to property. Special thanks are due the American Smelting and Refining Co. for permission to sample the open-pit mine under their lease. 


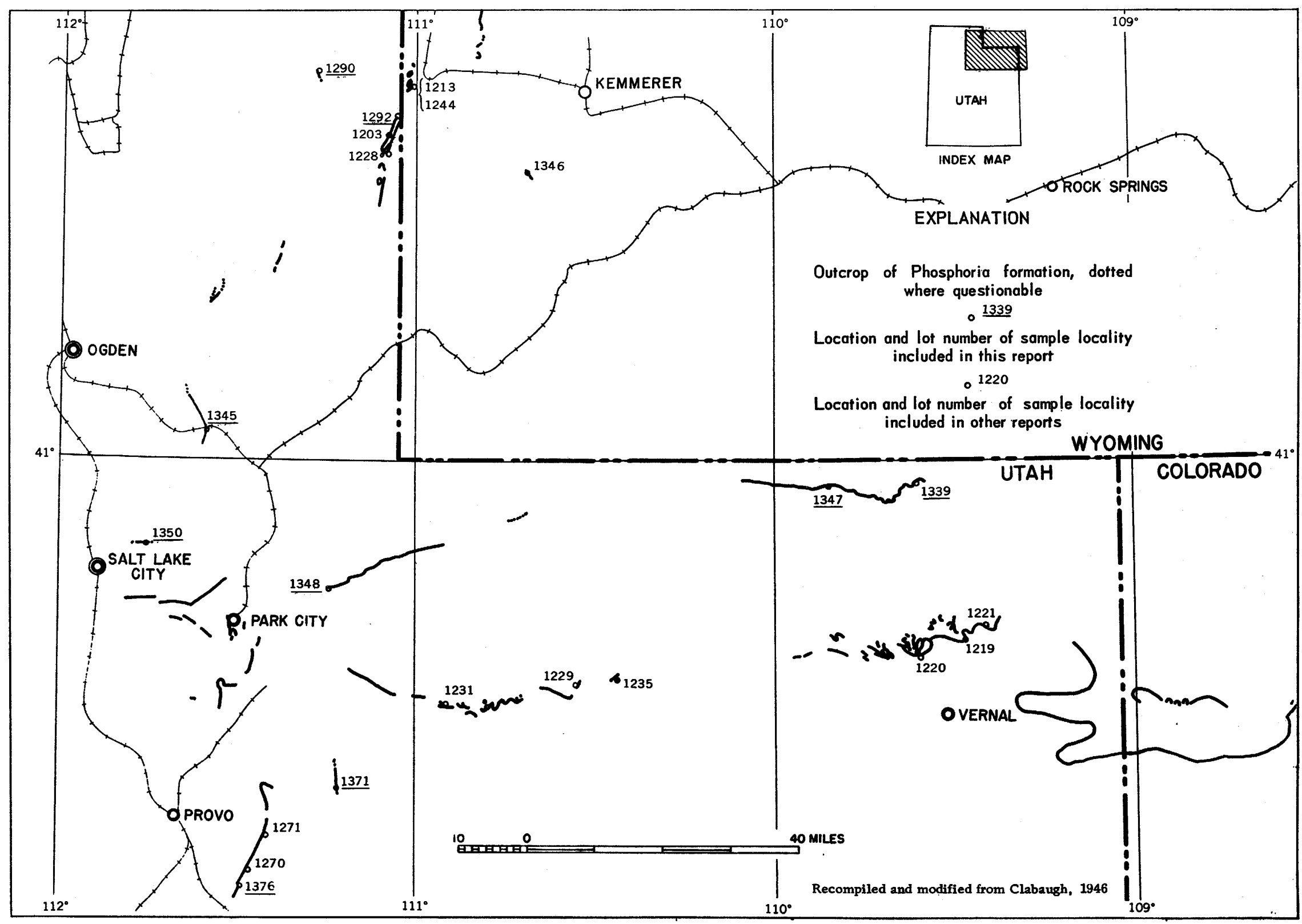

Figure 1. -Outcrops of the Phosphoria and Park City formations in Utah and localities sampled. 


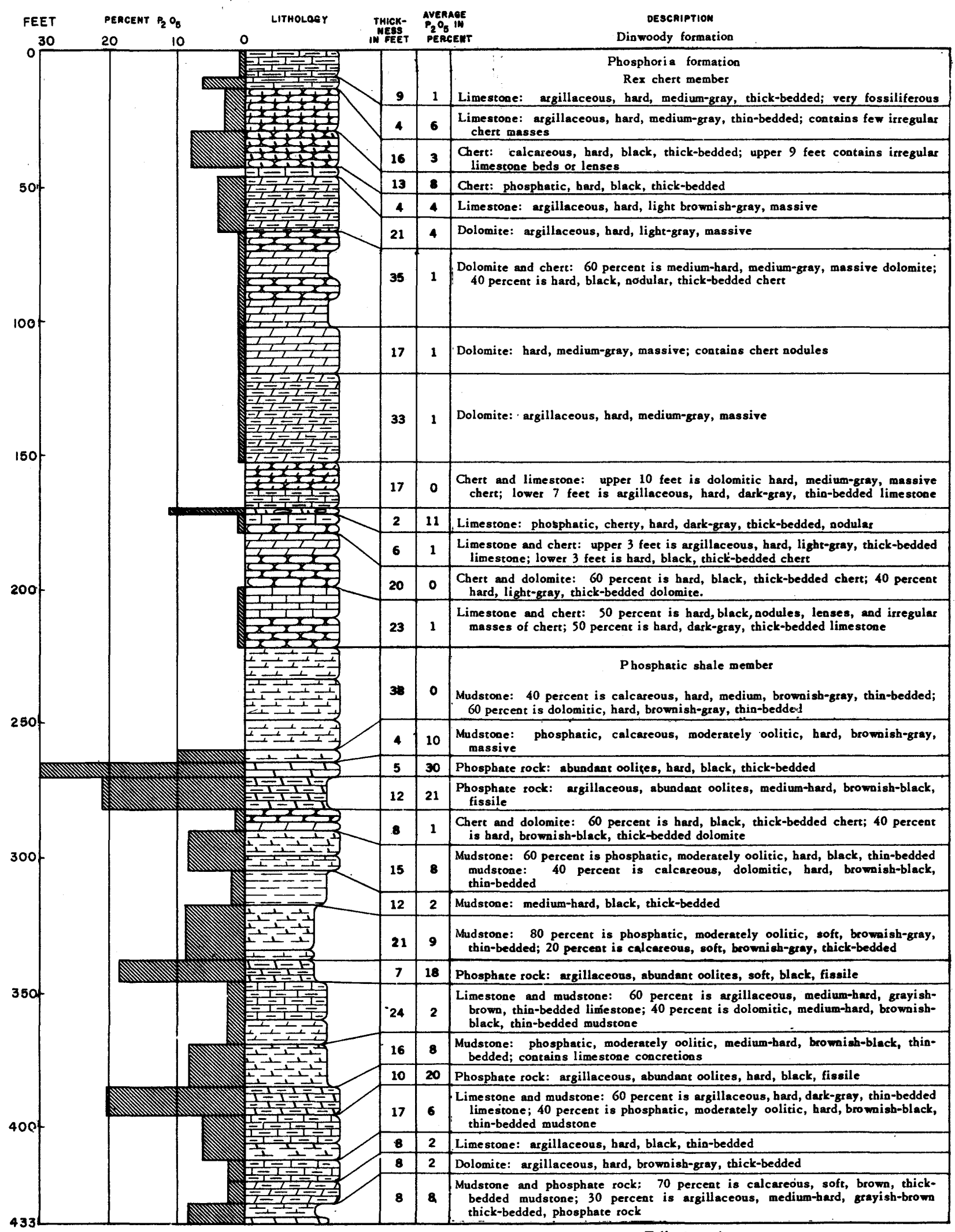

\author{
Wells formation
}

Figure 2. -Generalized section of the Phosphoria formation at Brazer Canyon, Utah, lot no. 1203. 


\section{STRATIGRAPHY OF THE PHOSPHORIA AND PARK CITY FORMATIONS IN UTAH}

The stratigraphy of the Permian Phosphoria formation in the Crawford Mountains of northeastern Utah, near the Idaho-Wyoming corner, is very similar to that in the adjacent states and described in companion reports (McKelvey and others, 1953a, 1953b). It consists of the phosphatic shale member, about 210 feet thick, and the Rex chert member, for which this is the type locality, about 220 feet thick. The upper shale member, present in the adjacent region to the north, is not well defined in this area. Here, as well a.s to the north, the Phosphoria formation overlies the Pennsylvanian Wells formation, the upper part of which corisists chiefly of cherty gray limestone with some thin phosphatic layers. The Phosphoria is overlain by the Triassic Dinwoody formation, consisting of limestone, calcareous siltstone, and sandstone. A generalized section of the Phosphoria formation at Brazer Canyon is shown in figure 2 .

Farther south, along both the north and south flanks of the Uinta Rarge and in the general area of the Wasatch Range, the Park City formation is the partial stratigraphic equivalent of the Phosphoria formation. At Park City, its type locality, it is about 590 feet thick and consists of a lower limestone member, which may be stratigraphically equivalent to the upper part of the Wells formation in southeastern Idaho; a middle shale member (phosphatic but containing no high-grade phosphate beds) probably equivalent in major part to the phosphatic shale of Idaho; and an upper limestone member, equivalent to the Rex chert member to the north. Eastward the lower limestone member thins out and the middle shale and upper limestone members thin, are more clastic, and finally tongue out into nonmarine redbeds in eastern Utah and western Colorado. Westward the formation thickens markedly, attaining a thickness of several thousand feet, and contains a. greater proportion of chemical precipitates.

More detailed correlations of the strata within Utah as well a.s between Utah and adjacent states will be discussed in subsequent pubiications.

\section{STRATIGRAPHIC SECTIONS}

Analytical data and abstracts of stratigraphic sections measured at nine localities follow. Their locations as well as the locations of sections previously reported (Smith and others, 1953) are shown in figure 1.

\section{SELECTED BIBLIOGRAPHY}

Clabaugh, P. S., 1946, Permian phosphate deposits of Montana, Idaho, Wyoming, and Utah: U. S. Geol. Survey, Strategic Minerals Inv. Prelim. Map 3-198.

McKelvey, V. E., Davidson, D. F., O'Malley, F.W. and Smith, L. E., 1953a, Stratigraphic sections of the Phosphoria formation in Idaho, 1947-48, pt. 1: U. S. Geol. Survey Circ. 208.

McKelvey, V. E., Smith, L. E., Hoppin, R. A., and Armstrong, F. C., 1953b, Stratigraphic sections of the Phosphoria formation in Wyoming, 1947-48: U. S. Geol. Survey Circ. 210.

Smith, L. E., Hosford, G. F., Sears, R. S., Sprouse, D. P., and Stewart, M. D., 1953, Stratigraphic sections of the Phosphoria formation in Utah, 1947-48: U. S. Geol. Survey Circ. 211. 
Laketown Canyon, Utah, lot 1290

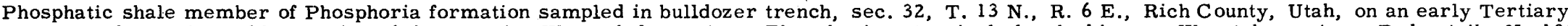
surface exhumed by. erosion of the covering Wasatch formation. Phosphoria extensively leached by pre-Wasatch erosion. Beds strike N. $10^{-} \mathrm{E}$. and dip $65^{\circ} \mathrm{W}$. Section measured by R. P. Sheldon, F. J. Anderson, and R. G. Waring and sampled by Waring in July 1949 . Samples analyzed for $\mathrm{P}_{2} \mathrm{O}_{5}$ and acid insoluble by U. S. Bureau of Mines laboratory, Albany, Oreg., and for other constituents by Trace Elements Section laboratory,

Washington, D. C.

\begin{tabular}{|c|c|c|c|c|c|c|c|c|c|c|}
\hline \multirow[b]{2}{*}{$\begin{array}{c}\text { Bed } \\
\text { no. }\end{array}$} & \multirow[b]{2}{*}{ Rock description } & \multirow{2}{*}{$\begin{array}{c}\text { Sample } \\
\text { no. }\end{array}$} & \multirow{2}{*}{$\begin{array}{c}\text { Thickness } \\
\text { (feet) }\end{array}$} & \multicolumn{5}{|c|}{ Chemical analyses (percent) } & \multirow{2}{*}{$\begin{array}{c}\text { Cumulative } \\
\text { thickness } \\
\text { (feet) }\end{array}$} & \multirow{2}{*}{$\begin{array}{l}\text { Thickness } \mathbf{x} \\
\text { percent } \mathrm{P}_{2} \mathrm{O}_{5} \\
\text { (cumulative) }\end{array}$} \\
\hline & & & & $\mathrm{P}_{2} \mathrm{O}_{5}$ & $\mathrm{Al}_{2} \mathrm{O}_{3}$ & $\mathrm{Fe}_{2} \mathrm{O}_{3}$ & $\begin{array}{l}\text { Loss on } \\
\text { ignition }\end{array}$ & $\begin{array}{c}\text { Acid } \\
\text { ins oluble }\end{array}$ & & \\
\hline
\end{tabular}

Rex chert member of Phosphoria formation-basal bed only

\begin{tabular}{|c|c|c|c|c|c|c|c|c|c|c|}
\hline $\mathrm{R}-1$ & Chert $\ldots$ & 3793- RPS & 0.8 & 1.2 & -- & -- & -- & 90.7 & 0.8 & -- \\
\hline \multicolumn{11}{|c|}{ Phosphatic shale member of Phosphoria formation } \\
\hline $\begin{array}{l}P-58 \\
P-57 \\
P-56 \\
P-55 \\
P-54\end{array}$ & $\begin{array}{l}\text { Mudstone, phosphatic } \\
\text { Mudstone } \\
\text { Mudstone } \\
\text { Mhosphate rock } \\
\text { rock }\end{array}$ & $\begin{array}{l}3792-\text { RPS } \\
3791-\text { RPS } \\
5190-\text { RPS } \\
3789-\text { RPS } \\
3787-\text { RPS }\end{array}$ & $\begin{array}{l}0.3 \\
2.6 \\
1.0 \\
1.0 \\
1.5\end{array}$ & $\begin{array}{r}11.0 \\
1.6 \\
5.8 \\
35.8 \\
9.0\end{array}$ & $\begin{array}{l}-- \\
-- \\
0.99 \\
8.2\end{array}$ & $\begin{array}{l}-- \\
-- \\
-\overline{0 .} \\
3.95\end{array}$ & $\begin{array}{l}-- \\
-- \\
-- \\
2.18 \\
4.26\end{array}$ & $\begin{array}{r}63.3 \\
75.8 \\
64.0 \\
4.0 \\
59.5\end{array}$ & $\begin{array}{l}0.3 \\
2.9 \\
3.9 \\
4.9 \\
6.4\end{array}$ & $\begin{array}{r}3.30 \\
7.46 \\
13.26 \\
49.06 \\
62.56\end{array}$ \\
\hline $\begin{array}{l}P-53 \\
P-52 \\
P-51 \\
P-50 \\
P-49\end{array}$ & $\begin{array}{l}\text { Phosphate rock } \\
\text { Mudstone, phosphatic } \\
\text { Phosphate rock } \\
\text { Phosphate rock } \\
\text { Phosphate rock }\end{array}$ & $\begin{array}{l}3877-\text { FJA } \\
3876-\text { FJA } \\
3875-\text { FJA } \\
3874-\text { FJA } \\
3873-\text { FJA }\end{array}$ & $\begin{array}{r}1.0 \\
.9 \\
1.9 \\
.5 \\
1.4\end{array}$ & $\begin{array}{l}31.0 \\
15.6 \\
33.5 \\
36.3 \\
31.4\end{array}$ & $\begin{array}{l}3.1 \\
7.0 \\
1.2 \\
1.3 \\
2.1\end{array}$ & $\begin{array}{r}1.93 \\
3.08 \\
.43 \\
.43 \\
.92\end{array}$ & $\begin{array}{l}4.10 \\
4.80 \\
3.62 \\
\text { 3. } 14 \\
\text { 3. } 74\end{array}$ & $\begin{array}{r}11.7 \\
42.9 \\
7.4 \\
3.5 \\
12.7\end{array}$ & $\begin{array}{r}7.4 \\
8.3 \\
10.2 \\
10.7 \\
12.1\end{array}$ & $\begin{array}{l}93.56 \\
107.60 \\
171.25 \\
189.40 \\
233.36\end{array}$ \\
\hline $\begin{array}{l}P-48 \\
P-47 \\
P-46 \\
P-45 \\
P-44\end{array}$ & $\begin{array}{l}\text { Phosphate rock } \\
\text { Phosphate rock } \\
\text { Phosphate rock, argillaceous } \\
\text { Phosphate rock, argillaceous } \\
\text { Mudstone and phosphate rock }\end{array}$ & $\begin{array}{l}3872-\text { FJA } \\
3871-\text { FJA } \\
3870-\text { FJA } \\
3869-\text { FJA } \\
3868-\text { FJA }\end{array}$ & $\begin{array}{r}1.3 \\
1.2 \\
.7 \\
.6 \\
.9\end{array}$ & $\begin{array}{l}36.5 \\
35.2 \\
27.5 \\
25.6 \\
21.5\end{array}$ & $\begin{array}{l}.92 \\
1.3 \\
4.3 \\
3.6 \\
6.0\end{array}$ & $\begin{array}{r}.63 \\
.37 \\
1.27 \\
.67 \\
2.08\end{array}$ & $\begin{array}{l}3.24 \\
3.78 \\
4.20 \\
5.80 \\
6.24\end{array}$ & $\begin{array}{l}2.2 \\
4.7 \\
20.7 \\
22.0 \\
27.2\end{array}$ & $\begin{array}{l}13.4 \\
14.6 \\
15.3 \\
15.9 \\
16.8\end{array}$ & $\begin{array}{l}280.81 \\
323.05 \\
342.30 \\
357.66 \\
377.01\end{array}$ \\
\hline $\begin{array}{l}P-43 \\
P-42 \\
P-41 \\
P-40 \\
P-39\end{array}$ & $\begin{array}{l}\text { Phosphate rock, argillaceous } \\
\text { Phosphate rock, argillaceous } \\
\text { Phosphate rock } \\
\text { Phosphate rock } \\
\text { Phosphate rock, argillaceous }\end{array}$ & $\begin{array}{l}3867-\text { FJA } \\
3866-\text { FJA } \\
3865-\text { FJA } \\
3864-\text { FJA } \\
3863-\text { FJA }\end{array}$ & $\begin{array}{r}.9 \\
1.0 \\
.8 \\
.7 \\
1.5\end{array}$ & $\begin{array}{l}16.4 \\
24.2 \\
29.8 \\
30.4 \\
22.3\end{array}$ & $\begin{array}{l}6.6 \\
4.5 \\
2.6 \\
2.7 \\
5.2\end{array}$ & $\begin{array}{r}2.60 \\
1.91 \\
1.56 \\
.71 \\
4.00\end{array}$ & $\begin{array}{l}7.02 \\
6.36 \\
6.84 \\
5.18 \\
4.44\end{array}$ & $\begin{array}{r}37.1 \\
22.7 \\
8.3 \\
11.3 \\
28.5\end{array}$ & $\begin{array}{l}17.7 \\
18.7 \\
19.5 \\
20.2 \\
21.7\end{array}$ & $\begin{array}{l}391.77 \\
415.97 \\
439.81 \\
461.09 \\
494.54\end{array}$ \\
\hline $\begin{array}{l}P-38 \\
P-37 \\
P-36 \\
P-35 \\
P-34\end{array}$ & $\begin{array}{l}\text { Phosphate rock, argillaceous } \\
\text { Phosphate rock, argillaceous } \\
\text { Phosphate rock, argillaceous } \\
\text { Mudstone, phosphatic } \\
\text { Mudstone, phosphatic }\end{array}$ & $\begin{array}{l}3862-\text { FJA } \\
3861-\text { FJA } \\
3860-\text { FJA } \\
3859-\text { FJA } \\
3858-\text { FJA }\end{array}$ & $\begin{array}{l}2.0 \\
1.0 \\
2.2 \\
1.5 \\
1.8\end{array}$ & $\begin{array}{l}21.9 \\
24.9 \\
19.2 \\
11.5 \\
13.5\end{array}$ & $\begin{array}{l}5.5 \\
4.8 \\
6.1 \\
-- \\
--\end{array}$ & $\begin{array}{l}2.14 \\
1.20 \\
3.15 \\
-- \\
--\end{array}$ & $\begin{array}{l}4.34 \\
4.34 \\
4.40 \\
-- \\
--\end{array}$ & $\begin{array}{l}32.2 \\
25.5 \\
36.9 \\
53.8 \\
51.8\end{array}$ & $\begin{array}{l}23.7 \\
24.7 \\
26.9 \\
28.4 \\
30.2\end{array}$ & $\begin{array}{l}538.34 \\
563.24 \\
605.48 \\
622.73 \\
647.03\end{array}$ \\
\hline$P-33$ & Mudstone & $3857-$ FJA & 2.5 & 1.3 & -- & - & -- & 83.7 & 32.7 & 650.28 \\
\hline
\end{tabular}


Laketown Canyon-Continued

\begin{tabular}{|c|c|c|c|c|c|c|c|c|c|c|}
\hline \multirow[b]{2}{*}{$\begin{array}{c}\text { Bed } \\
\text { no. }\end{array}$} & \multirow[b]{2}{*}{ Rock description } & \multirow{2}{*}{$\begin{array}{l}\text { Sample } \\
\text { no. }\end{array}$} & \multirow{2}{*}{$\begin{array}{l}\text { Thickness } \\
\text { (feet) }\end{array}$} & \multicolumn{5}{|c|}{ Chemical analyses (percent) } & \multirow{2}{*}{$\begin{array}{c}\text { Cumulative } \\
\text { thickness } \\
\text { (feet) }\end{array}$} & \multirow{2}{*}{$\begin{array}{l}\text { Thickness } x \\
\text { percent } \mathrm{P}_{2} \mathrm{O}_{5} \\
\text { (cumulative) }\end{array}$} \\
\hline & & & & $\mathrm{P}_{2} \mathrm{O}_{5}$ & $\mathrm{Al}_{2} \mathrm{O}_{3}$ & $\mathrm{Fe}_{2} \mathrm{O}_{3}$ & $\begin{array}{l}\text { Loss on } \\
\text { ignition }\end{array}$ & $\begin{array}{l}\text { Acid } \\
\text { insoluble }\end{array}$ & & \\
\hline $\begin{array}{l}P-32 \\
P-31 \\
P-30 \\
P-29 \\
P-28\end{array}$ & $\begin{array}{l}\text { Mudstone, phosphatic } \\
\text { Phosphate rock } \\
\text { Mudstone } \\
\text { Mudstone } \\
\text { Phosphate rock, argillaceous }\end{array}$ & $\begin{array}{l}3856-\text { FJA } \\
3855-\text { FJA } \\
\text { 3854- FJA } \\
\text { 3853- FJA } \\
\text { 3852- FJA }\end{array}$ & $\begin{array}{l}1.1 \\
1.2 \\
1.6 \\
3.5 \\
1.7\end{array}$ & $\begin{array}{r}11.3 \\
33.2 \\
6.9 \\
2.5 \\
27.3\end{array}$ & $\begin{array}{l}-- \\
-- \\
-- \\
--\end{array}$ & $\begin{array}{l}-- \\
-- \\
-- \\
-- \\
--\end{array}$ & $\begin{array}{l}-- \\
-- \\
-- \\
--\end{array}$ & $\begin{array}{r}54.4 \\
8.3 \\
66.1 \\
80.0 \\
18.3\end{array}$ & $\begin{array}{l}33.8 \\
35.0 \\
36.6 \\
40.1 \\
41.8\end{array}$ & $\begin{array}{l}662.71 \\
702.55 \\
713.59 \\
722.34 \\
768.75\end{array}$ \\
\hline $\begin{array}{l}P-27 \\
P-26\end{array}$ & $\begin{array}{l}\text { Mudstone } \\
\text { Phosphate rock }\end{array}$ & $\begin{array}{l}3851-\text { FJA } \\
3772-\text { RPS }\end{array}$ & $\begin{array}{l}1.8 \\
.85\end{array}$ & $\begin{array}{r}5.3 \\
27.7\end{array}$ & -- & -- & -- & $\begin{array}{l}69.9 \\
15.0\end{array}$ & $\begin{array}{l}43.6 \\
44.45\end{array}$ & $\begin{array}{l}778.29 \\
801.84\end{array}$ \\
\hline$P-25$ & Mudstone & - & $?$ & -- & -- & -- & -- & -- & -- & -- \\
\hline $\begin{array}{l}P-24 \\
P-23\end{array}$ & $\begin{array}{l}\text { Mudstone, phosphatic } \\
\text { Phosphate rock }\end{array}$ & $\begin{array}{l}3771-\text { RPS } \\
3770-\text { RPS }\end{array}$ & $\begin{array}{l}1.5 \\
1.0\end{array}$ & $\begin{array}{l}10.5 \\
29.5\end{array}$ & -- & -- & -- & $\begin{array}{l}54.6 \\
13.7\end{array}$ & $\begin{array}{l}45.95 \\
46.95\end{array}$ & $\begin{aligned} * 15.75 \\
45.25\end{aligned}$ \\
\hline $\begin{array}{l}P-22 \\
P-21 \\
P-20 \\
P-19 \\
P-18\end{array}$ & $\begin{array}{l}\text { Phosphate rock, argillaceous } \\
\text { Mudstone, phosphatic } \\
\text { Phosphate rock, argillaceous } \\
\text { Phosphate rock } \\
\text { Phosphate rock, argillaceous }\end{array}$ & $\begin{array}{l}3769-\text { RPS } \\
3768-\text { RPS } \\
3767-\text { RPS } \\
3788-\text { RPS } \\
3766-\text { RPS }\end{array}$ & $\begin{array}{r}1.6 \\
2.0 \\
2.0 \\
1.6 \\
.9\end{array}$ & $\begin{array}{r}19.1 \\
9.7 \\
23.1 \\
28.3 \\
19.8\end{array}$ & $\begin{array}{l}-- \\
-- \\
-- \\
--\end{array}$ & $\begin{array}{l}-- \\
-- \\
-- \\
--\end{array}$ & $\begin{array}{l}-- \\
-- \\
-- \\
--\end{array}$ & $\begin{array}{l}32.8 \\
58.0 \\
30.7 \\
16.0 \\
38.6\end{array}$ & $\begin{array}{l}48.55 \\
50.55 \\
52.55 \\
54.15 \\
55.05\end{array}$ & $\begin{array}{r}75.81 \\
95.21 \\
141.41 \\
186.69 \\
204.51\end{array}$ \\
\hline $\begin{array}{l}P-17 \\
P-16 \\
P-15 \\
P-14 \\
P-13\end{array}$ & $\begin{array}{l}\text { Mudstone } \\
\text { Mudstone } \\
\text { Mhosphate rock } \\
\text { Mudstone }\end{array}$ & $\begin{array}{l}3765-\text { RPS } \\
3764-\text { RPS } \\
3763-\text { RPS } \\
3786-\text { RPS } \\
3785-\text { RPS }\end{array}$ & $\begin{array}{l}1.6 \\
4.0 \\
1.0 \\
5.0 \\
5.0\end{array}$ & $\begin{array}{r}3.8 \\
1.1 \\
34.5 \\
4.1 \\
3.7\end{array}$ & $\begin{array}{l}-- \\
-- \\
-- \\
--\end{array}$ & $\begin{array}{l}-- \\
-- \\
-- \\
--\end{array}$ & $\begin{array}{l}-- \\
-- \\
-- \\
--\end{array}$ & $\begin{array}{r}76.4 \\
81.5 \\
6.8 \\
71.6 \\
69.7\end{array}$ & $\begin{array}{l}56.65 \\
60.65 \\
61.65 \\
66.65 \\
71.65\end{array}$ & $\begin{array}{l}210.59 \\
214.99 \\
249.49 \\
269.99 \\
288.49\end{array}$ \\
\hline $\begin{array}{l}P-12 \\
P-11 \\
P-10 \\
P-9 \\
P-8\end{array}$ & $\begin{array}{l}\text { Mudstone } \\
\text { Phosphate rock and mudstone } \\
\text { Mudstore, phosphatic } \\
\text { Phosphate rock, argillaceous } \\
\text { Phosphate rock and mudstone }\end{array}$ & $\begin{array}{l}3784-\text { RPS } \\
3783-\text { RPS } \\
3774-\text { RPS } \\
3773-\text { RPS } \\
3832-\text { RGW }\end{array}$ & $\begin{array}{r}4.5 \\
.5 \\
.8 \\
.7 \\
2.3\end{array}$ & $\begin{array}{r}6.7 \\
23.9 \\
17.3 \\
17.6 \\
19.1\end{array}$ & $\begin{array}{l}6.2 \\
6.9 \\
6.2 \\
6.7\end{array}$ & $\begin{array}{l}-. \\
2.52 \\
2.76 \\
2.43 \\
2.37\end{array}$ & $\begin{array}{l}-\infty \\
4.04 \\
3.68 \\
4.30 \\
4.18\end{array}$ & $\begin{array}{l}62.2 \\
28.0 \\
44.0 \\
41.4 \\
39.1\end{array}$ & $\begin{array}{l}76.15 \\
76.65 \\
77.45 \\
78.15 \\
80.45\end{array}$ & $\begin{array}{l}318.64 \\
330.59 \\
344.43 \\
356.75 \\
400.68\end{array}$ \\
\hline $\begin{array}{l}P-7 \\
P-6 \\
P-5 \\
P-4 \\
P-3\end{array}$ & $\begin{array}{l}\text { Phosphate rock, argillaceous } \\
\text { Mudstone } \\
\text { Phosphate rock, argillaceous } \\
\text { Phosphate rock, argillaceous } \\
\text { Phosphate rock, argillaceous }\end{array}$ & $\begin{array}{l}3831-\text { RGW } \\
3830-\text { RGW } \\
3829-\text { RGW } \\
3828-\text { RGW } \\
3827-\text { RGW }\end{array}$ & $\begin{array}{r}1.7 \\
.6 \\
1.5 \\
1.0 \\
2.4\end{array}$ & $\begin{array}{r}22.7 \\
7.5 \\
18.5 \\
23.1 \\
27.6\end{array}$ & $\begin{array}{r}5.1 \\
12 . \\
6.9 \\
4.9 \\
4.4\end{array}$ & $\begin{array}{l}1.58 \\
7.15 \\
3.88 \\
3.61 \\
1.54\end{array}$ & $\begin{array}{l}5.46 \\
6.58 \\
5.58 \\
4.90 \\
5.08\end{array}$ & $\begin{array}{l}28.2 \\
53.9 \\
34.4 \\
25.4 \\
16.2\end{array}$ & $\begin{array}{l}82.15 \\
82.75 \\
84.25 \\
85.25 \\
87.65\end{array}$ & $\begin{array}{l}439.27 \\
443.77 \\
471.52 \\
494.62 \\
560.86\end{array}$ \\
\hline $\begin{array}{l}P-2 \\
P-1\end{array}$ & $\begin{array}{l}\text { Phosphate rock and mudstone } \\
\text { Phosphate rock, argillaceous }\end{array}$ & $\begin{array}{l}\text { 3826-RGW } \\
\text { 3825-RGW }\end{array}$ & $\begin{array}{l}1.2 \\
1.5\end{array}$ & $\begin{array}{l}19.1 \\
18.7\end{array}$ & $\begin{array}{l}6.2 \\
6.2\end{array}$ & $\begin{array}{l}3.83 \\
6.30\end{array}$ & $\begin{array}{l}4.80 \\
4.80\end{array}$ & $\begin{array}{l}35.0 \\
35.0\end{array}$ & $\begin{array}{r}88.85 \\
* * 90.35\end{array}$ & $\begin{array}{r}583.78 \\
* * 611.83\end{array}$ \\
\hline \multicolumn{11}{|c|}{ Wells formation - upper part only } \\
\hline $\begin{array}{l}C w-1 \\
C w-2 \\
C w-3 \\
C w-4\end{array}$ & $\begin{array}{l}\text { Mudstone } \\
\text { Sandstone, cherty } \\
\text { Sandstone, cherty } \\
\text { Sandstone, cherty }\end{array}$ & $\begin{array}{l}3824-\text { RGW } \\
3823-\text { RGW } \\
3822-\text { RGW } \\
3821-\text { RGW }\end{array}$ & $\begin{array}{l}1.3 \\
1.2 \\
2.7 \\
1.4\end{array}$ & $\begin{array}{l}2.2 \\
2.0 \\
1.3 \\
1.3\end{array}$ & $\begin{array}{l}-- \\
-- \\
--\end{array}$ & $\begin{array}{l}-- \\
-- \\
--\end{array}$ & $\begin{array}{l}-- \\
-- \\
--\end{array}$ & $\begin{array}{l}83.0 \\
86.2 \\
86.2 \\
84.9\end{array}$ & $\begin{array}{l}1.3 \\
2.5 \\
5.2 \\
6.6\end{array}$ & $\begin{array}{r}2.86 \\
5.26 \\
8.77 \\
10.59\end{array}$ \\
\hline
\end{tabular}

* Cumulative data incomplete because of missing information. Cumulatives start from zero after interruption.

* Note incompleteness of cumulative data. 


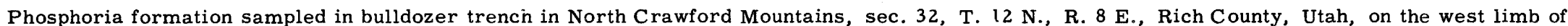
the Crawford syncline. Section measured by V. E. McKelvey, R. G. Waring, M. A. Warner, and R. A. Smart and sampled by Waring,

Warner, and Smart in July 1949. Samples analyzed for $\mathrm{P}_{2} \mathrm{O}_{5}$ and acid insoluble by U. S. Bureau of Mines laboratory, Albany, Oreg., and for other constituents by Trace Elements Section laboratory, Washington, D. C.

\begin{tabular}{|c|c|c|c|c|c|c|c|c|c|c|}
\hline \multirow[b]{2}{*}{$\begin{array}{l}\text { Bed } \\
\text { no. }\end{array}$} & \multirow[b]{2}{*}{ Rock description } & \multirow{2}{*}{$\begin{array}{c}\text { Sample } \\
\text { no. }\end{array}$} & \multirow{2}{*}{$\begin{array}{c}\text { Thickness } \\
\text { (feet) }\end{array}$} & \multicolumn{5}{|c|}{ Chemical analyses (percent) } & \multirow{2}{*}{$\begin{array}{l}\text { Cumulative } \\
\text { thickness } \\
\text { (feet) }\end{array}$} & \multirow{2}{*}{$\begin{array}{l}\text { Thickness } \mathrm{x} \\
\text { percent } \mathrm{P}_{2} \mathrm{O}_{5} \\
\text { (cumulative) }\end{array}$} \\
\hline & & & & $\mathrm{P}_{2} \mathrm{O}_{5}$ & $\mathrm{Al}_{2} \mathrm{O}_{3}$ & $\mathrm{Fe}_{2} \mathrm{O}_{3}$ & $\begin{array}{l}\text { Loss on } \\
\text { ignition }\end{array}$ & $\begin{array}{c}\text { Acid } \\
\text { insoluble }\end{array}$ & & \\
\hline \multicolumn{11}{|c|}{ Phosphatic shale member of Phosphoria formation } \\
\hline $\begin{array}{l}P-104 \\
P-103 \\
P-102 \\
P-101 \\
P-100\end{array}$ & $\begin{array}{l}\text { Mudstone, carbonatic } \\
\text { Mudstone, carbonatic } \\
\text { Mudstone, carbonatic } \\
\text { Phosphate rock, argillaceous } \\
\text { Carbonate rock, argillaceous, and } \\
\text { argillaceous phosphate rock }\end{array}$ & $\begin{array}{l}\text { 3843-MAW } \\
3842-\text { MAW } \\
\text { 3933-VEM } \\
3932-\text { VEM } \\
\text { 3931-VEM }\end{array}$ & $\begin{array}{l}5.7 \\
5.9 \\
2.8 \\
1.4 \\
1.5\end{array}$ & $\begin{array}{r}0.6 \\
.6 \\
17.7 \\
9.1\end{array}$ & $\begin{array}{l}-- \\
-- \\
--\end{array}$ & $\begin{array}{l}-- \\
-- \\
-- \\
--\end{array}$ & $\begin{array}{l}-- \\
-- \\
-- \\
--\end{array}$ & $\begin{array}{l}57.2 \\
52.7 \\
63.0 \\
35.7 \\
32.0\end{array}$ & $\begin{array}{l}5.7 \\
11.6 \\
14.4 \\
15.8 \\
17.3\end{array}$ & $\begin{array}{r}3.42 \\
6.96 \\
8.92 \\
32.86 \\
46.51\end{array}$ \\
\hline $\begin{array}{l}P=99 \\
P=98 \\
P=97 \\
P=96\end{array}$ & $\begin{array}{l}\text { Mudstone, phosphatic } \\
\text { Phosphate rock } \\
\text { Phosphate rock } \\
\text { Phosphate rock, argillaceous, and }\end{array}$ & $\begin{array}{l}3930-V E M \\
3929-\text { VEM } \\
3928-\text { VEM }\end{array}$ & $\begin{array}{r}.9 \\
1.8 \\
1.4\end{array}$ & $\begin{array}{r}8.3 \\
32.3 \\
32.8\end{array}$ & $\begin{array}{r}-- \\
0.74 \\
.49\end{array}$ & $\begin{array}{r}-. \\
0.67 \\
.43\end{array}$ & $\begin{array}{l}-- \\
5.86 \\
6.44\end{array}$ & $\begin{array}{r}51.3 \\
3.7 \\
2.0\end{array}$ & $\begin{array}{l}18.2 \\
20.0 \\
21.4\end{array}$ & $\begin{array}{r}53.98 \\
112.12 \\
158.04\end{array}$ \\
\hline & $\begin{array}{l}\text { phosphatic mudstone } \\
\text { Phosphate rock }\end{array}$ & $\begin{array}{l}\text { 3927- VEM } \\
3926 \text { - VEM }\end{array}$ & $\begin{array}{r}.5 \\
1.2\end{array}$ & $\begin{array}{l}19.9 \\
30.3\end{array}$ & $\begin{array}{l}3.9 \\
.49\end{array}$ & $\begin{array}{r}1.21 \\
.50\end{array}$ & $\begin{array}{l}7.40 \\
9.04\end{array}$ & $\begin{array}{r}29.8 \\
2.4\end{array}$ & $\begin{array}{l}21.9 \\
23.1\end{array}$ & $\begin{array}{l}167.99 \\
204.35\end{array}$ \\
\hline $\begin{array}{l}P-94 \\
P-93 \\
P-92 \\
P-91 \\
P-90\end{array}$ & $\begin{array}{l}\text { Phosphate rock, phosphatic mudstone, } \\
\text { and carbonatic phosphate rock } \\
\text { Phosphate rock } \\
\text { Phosphate rock } \\
\text { Phosphate rock, argillaceous } \\
\text { Mudstone, carbonatic }\end{array}$ & $\begin{array}{l}3925-\text { VEM } \\
3924-\text { VEM } \\
3923-\text { VEM } \\
3922-\text { VEM } \\
3921-\text { VEM }\end{array}$ & $\begin{array}{r}.9 \\
1.7 \\
.9 \\
1.3 \\
2.8\end{array}$ & $\begin{array}{r}27.3 \\
29.4 \\
27.7 \\
19.0 \\
2.6\end{array}$ & $\begin{array}{l}2.5 \\
1.4 \\
2.7 \\
3.4 \\
6.1\end{array}$ & $\begin{array}{r}1.20 \\
.38 \\
.82 \\
1.27 \\
1.94\end{array}$ & $\begin{array}{r}5.92 \\
6.78 \\
4.80 \\
9.26 \\
19.40\end{array}$ & $\begin{array}{r}15.1 \\
9.3 \\
19.7 \\
27.0 \\
47.3\end{array}$ & $\begin{array}{l}24.0 \\
25.7 \\
26.6 \\
27.9 \\
30.7\end{array}$ & $\begin{array}{l}228.92 \\
278.90 \\
312.14 \\
331.14 \\
338.42\end{array}$ \\
\hline $\begin{array}{l}P-89 \\
P-88 \\
P-87 \\
P-86 \\
P-85\end{array}$ & $\begin{array}{l}\text { Phosphate rock } \\
\text { Phosphate rock } \\
\text { Phosphate rock, argillaceous } \\
\text { Mudstone, phosphatic } \\
\text { Phosphate rock }\end{array}$ & $\begin{array}{l}\text { 3841-MAW } \\
\text { 3840-MAW } \\
3839-\text { MAW } \\
3838-\text { MAW } \\
\text { 3837-MAW }\end{array}$ & $\begin{array}{r}2.0 \\
.9 \\
1.8 \\
.5 \\
1.1\end{array}$ & $\begin{array}{r}25.6 \\
27.2 \\
22.0 \\
9.5 \\
27.7\end{array}$ & $\begin{array}{l}3.3 \\
2.1 \\
4.2 \\
6.2 \\
1.7\end{array}$ & $\begin{array}{l}1.00 \\
3.63 \\
1.82 \\
2.45 \\
1.60\end{array}$ & $\begin{array}{l}4.34 \\
4.38 \\
3.58 \\
8.50 \\
6.00\end{array}$ & $\begin{array}{l}23.4 \\
18.7 \\
32.7 \\
52.8 \\
13.7\end{array}$ & $\begin{array}{l}32.7 \\
33.6 \\
35.4 \\
35.9 \\
37.0\end{array}$ & $\begin{array}{l}389.62 \\
414.10 \\
453.70 \\
458.45 \\
488.92\end{array}$ \\
\hline $\begin{array}{l}P-84 \\
P-83 \\
P-82\end{array}$ & $\begin{array}{l}\text { Carbonate rock } \\
\text { Mudstone, cherty } \\
\text { Phosphate rock, argillaceous, }\end{array}$ & $\begin{array}{l}\text { 3836-MAW } \\
\text { 3835-MAW }\end{array}$ & $\begin{array}{l}1.2 \\
1.2\end{array}$ & $\begin{array}{l}.7 \\
.9\end{array}$ & -- & -- & -- & $\begin{array}{l}12.3 \\
81.1\end{array}$ & $\begin{array}{l}38.2 \\
39.4\end{array}$ & $\begin{array}{l}489.76 \\
490.84\end{array}$ \\
\hline $\begin{array}{l}P=81 \\
P-80\end{array}$ & $\begin{array}{l}\text { carbonatic } \\
\text { Mudstone, carbonatic } \\
\text { Mudstone, carbonatic }\end{array}$ & $\begin{array}{l}\text { 3834-MAW } \\
\text { 3833-MAW } \\
\text { 3986- RGW }\end{array}$ & $\begin{array}{l}1.4 \\
3.0 \\
1.9\end{array}$ & $\begin{array}{r}17.3 \\
2.3 \\
4.0\end{array}$ & $\ddot{--}$ & $\begin{array}{l}-- \\
--\end{array}$ & $\begin{array}{l}-- \\
-- \\
--\end{array}$ & $\begin{array}{l}+9.1 \\
44.0 \\
55.3\end{array}$ & $\begin{array}{l}40.8 \\
43.8 \\
45.7\end{array}$ & $\begin{array}{l}515.06 \\
521.96 \\
529.56\end{array}$ \\
\hline $\begin{array}{l}P-79 \\
P-78 \\
P-77\end{array}$ & $\begin{array}{l}\text { Mudstone } \\
\text { Mudstone and phosphate rock } \\
\text { Carbonate rock, argillaceous }\end{array}$ & $\begin{array}{l}\text { 3985- RGW } \\
\text { 3984- RGW } \\
\text { 3983- RGW }\end{array}$ & $\begin{array}{l}.5 \\
.7 \\
.6\end{array}$ & $\begin{array}{r}2.3 \\
14.9 \\
.5\end{array}$ & $\begin{array}{ll}-- \\
--\end{array}$ & $\begin{array}{ll}-- \\
--\end{array}$ & -- & $\begin{array}{l}75.5 \\
38.3 \\
22.3\end{array}$ & $\begin{array}{l}46.2 \\
46.9 \\
47.5\end{array}$ & $\begin{array}{l}530.71 \\
541.14 \\
541.44\end{array}$ \\
\hline
\end{tabular}


North C rawford-Continued

\begin{tabular}{|c|c|c|c|c|c|c|c|c|c|c|}
\hline \multirow{2}{*}{$\begin{array}{c}\text { Bed } \\
\text { no. }\end{array}$} & \multirow[b]{2}{*}{ Rock description } & \multirow{2}{*}{$\begin{array}{l}\text { Sample } \\
\text { no. }\end{array}$} & \multirow{2}{*}{$\begin{array}{c}\text { Thickness } \\
\text { (feet) }\end{array}$} & \multicolumn{5}{|c|}{ Chemical analyses (percent) } & \multirow{2}{*}{$\begin{array}{l}\text { Cumulative } \\
\text { thickness } \\
\text { (feet) }\end{array}$} & \multirow{2}{*}{$\begin{array}{l}\text { Thickness } \mathrm{x} \\
\text { percent } \mathrm{P}_{2} \mathrm{O}_{5} \\
\text { (cumulative) }\end{array}$} \\
\hline & & & & $\mathrm{P}_{2} \mathrm{O}_{5}$ & $\mathrm{Al}_{2} \mathrm{O}_{3}$ & $\mathrm{Fe}_{2} \mathrm{O}_{3}$ & $\begin{array}{l}\text { Loss on } \\
\text { ignition }\end{array}$ & $\begin{array}{l}\text { Acid } \\
\text { insoluble }\end{array}$ & & \\
\hline $\begin{array}{l}P-76 \\
P-75 \\
P-74 \\
P-73\end{array}$ & $\begin{array}{l}\text { Mudstone, phosphatic, carbonatic } \\
\text { Mudstone, carbonatic } \\
\text { Phosphate rock, argillaceous } \\
\text { Carbonate rock, argillaceous, }\end{array}$ & $\begin{array}{l}\text { 3982-RGW } \\
3981-\mathrm{RGW} \\
3980-\mathrm{RGW}\end{array}$ & $\begin{array}{l}0.5 \\
1.8 \\
1.1\end{array}$ & $\begin{array}{r}10.3 \\
1.7 \\
25.6\end{array}$ & $\begin{array}{ll}-- \\
--\end{array}$ & $\begin{array}{ll}-- \\
--\end{array}$ & $\begin{array}{ll}-- \\
--\end{array}$ & $\begin{array}{l}36.3 \\
52.3 \\
16.3\end{array}$ & $\begin{array}{l}48.0 \\
49.8 \\
50.9\end{array}$ & $\begin{array}{l}546.59 \\
549.65 \\
577.81\end{array}$ \\
\hline$P-72$ & Carbonate rock & $\begin{array}{l}\text { 3979-RGW } \\
\text { 3940-VEM }\end{array}$ & $\begin{array}{l}1.6 \\
3.2\end{array}$ & $\begin{array}{r}8.5 \\
.4\end{array}$ & -- & -- & -- & $\begin{array}{r}24.5 \\
6.3\end{array}$ & $\begin{array}{l}52.5 \\
55.7\end{array}$ & $\begin{array}{l}591.41 \\
592.69\end{array}$ \\
\hline $\begin{array}{l}P-71 \\
P-70 \\
P-69 \\
P-68 \\
P-67\end{array}$ & $\begin{array}{l}\text { Mudstone, carbonatic } \\
\text { Mudstone } \\
\text { Mudstone } \\
\text { Mudstone }\end{array}$ & $\begin{array}{l}3939-V E M \\
3938-V E M \\
3937-V E M \\
3936-V E M \\
3935-V E M\end{array}$ & $\begin{array}{r}.3 \\
.3 \\
.5 \\
.8 \\
1.0\end{array}$ & $\begin{array}{r}5.2 \\
.4 \\
.2 \\
.2 \\
5.7\end{array}$ & $\begin{array}{l}-- \\
-- \\
-- \\
--\end{array}$ & $\begin{array}{l}-- \\
-- \\
-- \\
--\end{array}$ & $\begin{array}{l}-- \\
-- \\
-- \\
--\end{array}$ & $\begin{array}{l}56.2 \\
78.9 \\
71.5 \\
75.3 \\
65.1\end{array}$ & $\begin{array}{l}56.0 \\
56.3 \\
56.8 \\
57.6 \\
58.6\end{array}$ & $\begin{array}{l}594.25 \\
594.37 \\
594.47 \\
594.63 \\
600.33\end{array}$ \\
\hline $\begin{array}{l}P-66 \\
P-65 \\
P-64 \\
P-63 \\
P-62\end{array}$ & $\begin{array}{l}\text { Mudstone, carbonatic } \\
\text { Mudstone, phosphatic } \\
\text { Mudstone, } \\
\text { Mudstone, carbonatic } \\
\text { Mudstone, phosphatic }\end{array}$ & $\begin{array}{l}3934-\text { VEM } \\
3848-\text { RAS } \\
3847-\text { RAS } \\
3846-\text { RAS } \\
3845-\text { RAS }\end{array}$ & $\begin{array}{r}2.2 \\
.6 \\
1.2 \\
1.9 \\
.6\end{array}$ & $\begin{array}{r}1.1 \\
11.4 \\
.9 \\
10.7\end{array}$ & $\begin{array}{l}-- \\
-- \\
-- \\
--\end{array}$ & $\begin{array}{l}-- \\
-- \\
-- \\
--\end{array}$ & $\begin{array}{l}-- \\
-- \\
-- \\
--\end{array}$ & $\begin{array}{l}52.8 \\
53.3 \\
77.3 \\
59.8 \\
50.2\end{array}$ & $\begin{array}{l}60.8 \\
61.4 \\
62.6 \\
64.5 \\
65.1\end{array}$ & $\begin{array}{l}602.75 \\
609.59 \\
610.67 \\
612.00 \\
618.48\end{array}$ \\
\hline $\begin{array}{l}{ }^{\infty} \mathrm{P}-61 \\
\mathrm{P}-60 \\
\mathrm{P}-59 \\
\mathrm{P}-58\end{array}$ & $\begin{array}{l}\text { Mudstone, phosphatic } \\
\text { Mudstone, phosphatic } \\
\text { Carbonate rock } \\
\text { Phosphate rock, argillaceous, }\end{array}$ & $\begin{array}{l}3844-\text { RAS } \\
3900-\text { RAS } \\
3899-\text { RAS }\end{array}$ & $\begin{array}{r}1.4 \\
1.8 \\
.8\end{array}$ & $\begin{array}{r}11.7 \\
10.1 \\
2.9\end{array}$ & -- & $\ddot{--}$ & -- & $\begin{array}{r}48.2 \\
50.2 \\
9.5\end{array}$ & $\begin{array}{l}66.5 \\
68.3 \\
69.1\end{array}$ & $\begin{array}{l}634.86 \\
653.04 \\
655.36\end{array}$ \\
\hline $\mathbf{P}-57$ & $\begin{array}{l}\text { carbonatic } \\
\text { Mudstone, carbonatic }\end{array}$ & $\begin{array}{l}3898-\text { RAS } \\
3897-\text { RAS }\end{array}$ & $\begin{array}{l}2.5 \\
1.6\end{array}$ & $\begin{array}{r}14.7 \\
5.1\end{array}$ & -- & -- & $\overline{--}$ & $\begin{array}{l}28.3 \\
47.5\end{array}$ & $\begin{array}{l}71.6 \\
73.2\end{array}$ & $\begin{array}{l}692.11 \\
700.27\end{array}$ \\
\hline $\begin{array}{l}P-56 \\
P-55 \\
P-54\end{array}$ & $\begin{array}{l}\text { Phosphate rock, argillaceous } \\
\text { Carbonate rock } \\
\text { Phosphate rock, argillaceous, }\end{array}$ & $\begin{array}{l}\text { 3896- RAS } \\
\text { 3895- RAS }\end{array}$ & $\begin{array}{r}1.0 \\
.6\end{array}$ & $\begin{array}{r}19.7 \\
2.8\end{array}$ & -- & -- & -- & $\begin{array}{r}33.0 \\
8.5\end{array}$ & $\begin{array}{l}74.2 \\
74.8\end{array}$ & $\begin{array}{l}719.97 \\
721.65\end{array}$ \\
\hline $\begin{array}{l}P-53 \\
P-52\end{array}$ & $\begin{array}{l}\text { Mudstone, phosphatic, carbonatic } \\
\text { Mudstone, phosphatic and argillaceous }\end{array}$ & $\begin{array}{l}\text { 3894- RAS } \\
\text { 3893- RAS }\end{array}$ & 1.8 & $\begin{array}{l}14.1 \\
10.4\end{array}$ & -- & $=-$ & -- & $\begin{array}{l}22.7 \\
35.8\end{array}$ & $\begin{array}{l}75.6 \\
77.4\end{array}$ & $\begin{array}{l}732.93 \\
751.65\end{array}$ \\
\hline & limestone - & 3892-RAS & 1.7 & 9.4 & -- & -- & -- & 49.0 & 79.1 & 767.63 \\
\hline $\begin{array}{l}P-51 \\
P-50 \\
P-49\end{array}$ & $\begin{array}{l}\text { Phosphate rock, argillaceous } \\
\text { Phosphate rock, argillaceous } \\
\text { Phosphate rock, argillaceous and }\end{array}$ & $\begin{array}{l}3891-\text { RAS } \\
3890-\text { RAS }\end{array}$ & $\begin{array}{l}2.7 \\
1.4\end{array}$ & $\begin{array}{l}18.3 \\
20.6\end{array}$ & -- & -- & -- & $\begin{array}{l}31.2 \\
20.9\end{array}$ & $\begin{array}{l}81.8 \\
83.2\end{array}$ & $\begin{array}{l}817.04 \\
845.88\end{array}$ \\
\hline $\begin{array}{l}P-48 \\
P-47\end{array}$ & $\begin{array}{l}\text { mudstone } \\
\text { Phosphate rock } \\
\text { Phosphate rock, argillaceous }\end{array}$ & $\begin{array}{l}\text { 3889- RAS } \\
3888-\text { RAS } \\
\text { 3978-RGW }\end{array}$ & $\begin{array}{l}1.1 \\
1.2 \\
1.0\end{array}$ & $\begin{array}{l}21.8 \\
33.1 \\
23.7\end{array}$ & $\begin{array}{l}-- \\
--\end{array}$ & -- & $\begin{array}{l}-- \\
--\end{array}$ & $\begin{array}{r}29.7 \\
9.3 \\
29.0\end{array}$ & $\begin{array}{l}84.3 \\
85.5 \\
86.5\end{array}$ & $\begin{array}{l}869.86 \\
909.58 \\
933.28\end{array}$ \\
\hline $\begin{array}{l}P-46 \\
P-45 \\
P-44\end{array}$ & $\begin{array}{l}\text { Mudstone, phosphatic } \\
\text { Carbonate rock } \\
\text { Mudstone }\end{array}$ & $\begin{array}{l}\text { 3977-RGW } \\
\text { 3976-RGW } \\
\text { 3975-RGW }\end{array}$ & $\begin{array}{l}.8 \\
1.3 \\
3.3\end{array}$ & $\begin{array}{r}14.3 \\
3.1 \\
6.9\end{array}$ & $\begin{array}{l}-- \\
--\end{array}$ & $\begin{array}{l}-- \\
-- \\
--\end{array}$ & $\begin{array}{l}-- \\
-- \\
--\end{array}$ & $\begin{array}{l}38.3 \\
18.8 \\
51.3\end{array}$ & $\begin{array}{l}87.3 \\
88.6 \\
91.9\end{array}$ & $\begin{array}{l}944.72 \\
948.75 \\
971.52\end{array}$ \\
\hline
\end{tabular}




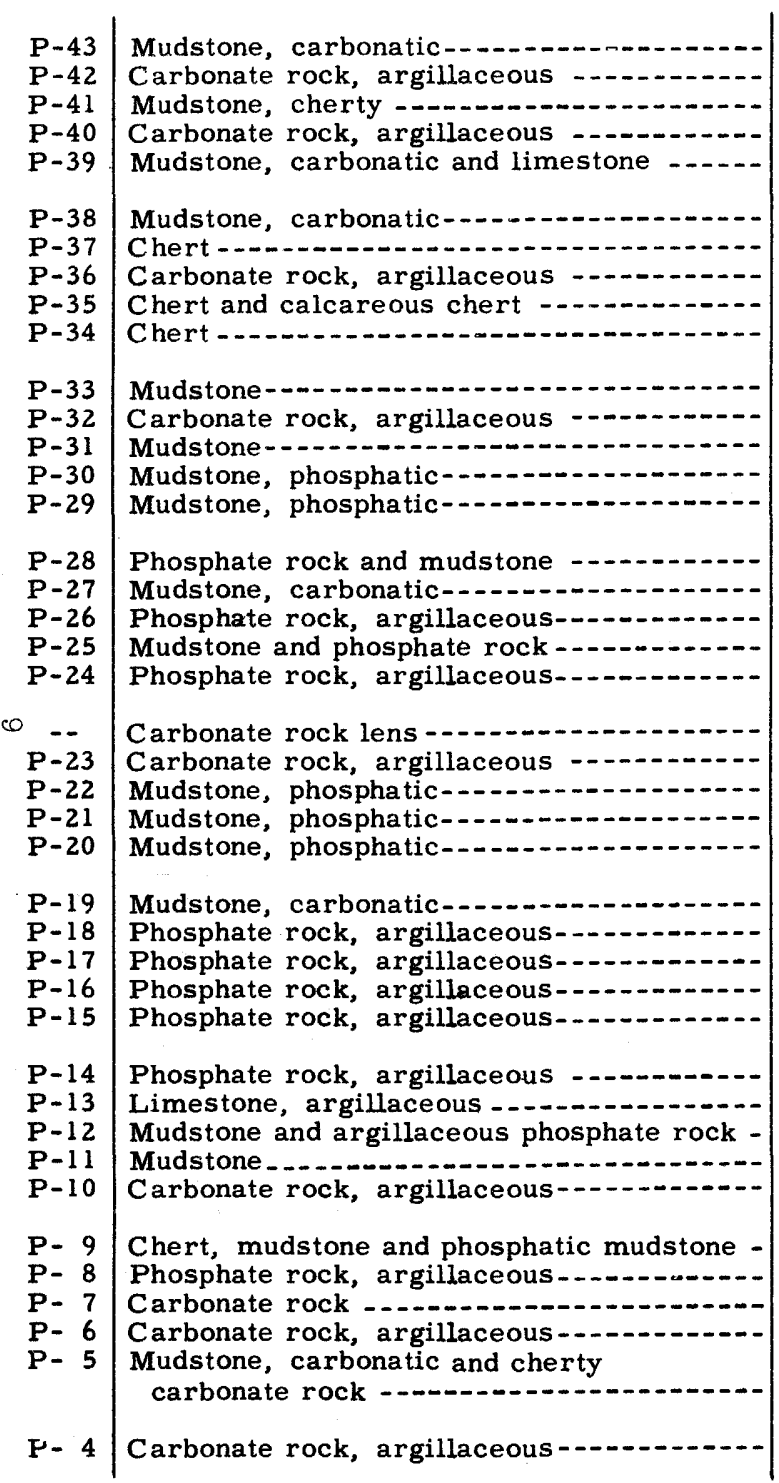

3974-RGW
$3973-\mathrm{RGW}$
$3972-\mathrm{RGW}$
$3971-\mathrm{RGW}$
$3970-\mathrm{RGW}$
$3969-\mathrm{RGW}$
$3968-\mathrm{RGW}$
$3967-\mathrm{RGW}$
$3966-\mathrm{RGW}$
$3965-\mathrm{RGW}$
3964-RGW
$3963-\mathrm{RGW}$
$3962-\mathrm{RGW}$
$3961-\mathrm{RGW}$
$3960-\mathrm{RGW}$
$3887-\mathrm{RAS}$
$3886-\mathrm{RAS}$
$3885-\mathrm{RAS}$
$3884-\mathrm{RAS}$
$3883-\mathrm{RAS}$
$3882-\mathrm{RAS}$
$3881-\mathrm{RAS}$
$3880-\mathrm{RAS}$
$3879-\mathrm{RAS}$
$3878-\mathrm{RAS}$
$3794-\mathrm{RAS}$
$3795-\mathrm{RAS}$
$3796-\mathrm{RAS}$
$3797-\mathrm{RAS}$
3798- RAS
3799- RAS
$3959-\mathrm{RGW}$
$3958-\mathrm{RGW}$
$3957-\mathrm{RGW}$
$3956-\mathrm{RGW}$
$3955-\mathrm{RGW}$
$3954-\mathrm{RGW}$
$3953-\mathrm{RGW}$
$3952-\mathrm{RGW}$
$3951-\mathrm{RGW}$
3951-RGW

\begin{tabular}{|c|c|c|c|}
\hline 4.7 & 1.6 & -- & -- \\
\hline $\begin{array}{l}1.3 \\
1.4\end{array}$ & 2.2 & -- & -- \\
\hline 1.1 & 1.6 & -. & $=$ \\
\hline .9 & .7 & -- & -- \\
\hline 1.1 & 29 & -- & -- \\
\hline & 3. 4 & -- & -- \\
\hline 2.0 & .8 & -- & -- \\
\hline .6 & 3.1 & -- & -- \\
\hline 1.1 & 4.7 & -- & -- \\
\hline & 1.5 & -. & -- \\
\hline .9 & $\begin{array}{l}4.5 \\
9.9\end{array}$ & $\because$ & -- \\
\hline 1.1 & 11.6 & - & -- \\
\hline 1.1 & 11.9 & -- & -- \\
\hline .0 & 1.2 & -- & -- \\
\hline 6 & 20.5 & -- & -- \\
\hline .5 & $\begin{array}{l}18.0 \\
22.3\end{array}$ & -- & $\because-$ \\
\hline (.7) & 8 & -- & -- \\
\hline 1.8 & 1.4 & -. & -- \\
\hline 6 & 14.1 & -- & 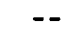 \\
\hline 1.4 & 14.1 & -- & -- \\
\hline & 13.2 & & - \\
\hline 1.4 & $\begin{array}{r}1.5 \\
\end{array}$ & $\overline{4}$ & $-\overline{20}$ \\
\hline $\begin{array}{l}1.9 \\
2.5\end{array}$ & $\begin{array}{l}27.2 \\
24.8\end{array}$ & $\begin{array}{r}2.4 \\
-\end{array}$ & 1.20 \\
\hline 1.2 & 25.1 & 2.3 & .42 \\
\hline & 28.9 & & \\
\hline 1.9 & 19.1 & -- & -- \\
\hline 1.2 & $\begin{array}{r}4.8 \\
19.7\end{array}$ & 1.2 & .26 \\
\hline${ }^{1}$ & 7,4 & - & - \\
\hline .7 & 7.2 & - & -- \\
\hline 8 & 8.1 & -- & $\ldots$ \\
\hline & 15.7 & -- & -- \\
\hline $\begin{array}{l}1.2 \\
1.2\end{array}$ & $\begin{array}{l}1.1 \\
3.4\end{array}$ & -- & -- \\
\hline 1.4 & 37 & & \\
\hline & & & \\
\hline 1.1 & 2.1 & - & -- \\
\hline
\end{tabular}

\begin{tabular}{l}
-- \\
-- \\
-- \\
-- \\
-- \\
-- \\
-- \\
-- \\
-- \\
-- \\
-- \\
-- \\
-- \\
-- \\
-- \\
-- \\
- \\
-- \\
-- \\
-- \\
-- \\
-- \\
3.38 \\
3.26 \\
3.20 \\
-- \\
25.18 \\
5.64 \\
-- \\
-- \\
-- \\
-- \\
-- \\
-- \\
-- \\
-- \\
\\
- \\
\hline
\end{tabular}


North Crawford-Continued

\begin{tabular}{|c|c|c|c|c|c|c|c|c|c|c|}
\hline \multirow[b]{2}{*}{$\begin{array}{c}\text { Bed } \\
\text { no. }\end{array}$} & \multirow[b]{2}{*}{ Rock description } & \multirow[b]{2}{*}{$\begin{array}{c}\text { Sample } \\
\text { no. }\end{array}$} & \multirow[b]{2}{*}{$\begin{array}{c}\text { Thickness } \\
\text { (feet) }\end{array}$} & \multicolumn{5}{|c|}{ Chemical analyses (percent) } & \multirow{2}{*}{$\begin{array}{c}\text { Cumulative } \\
\text { thickness } \\
\text { (feet) }\end{array}$} & \multirow{2}{*}{$\begin{array}{l}\text { Thickness } x \\
\text { percent } \mathrm{P}_{2} \mathrm{O}_{5} \\
\text { (cumulative) }\end{array}$} \\
\hline & & & & $\mathrm{P}_{2} \mathrm{O}_{5}$ & $\mathrm{Al}_{2} \mathrm{O}_{3}$ & $\mathrm{Fe}_{2} \mathrm{O}_{3}$ & $\begin{array}{l}\text { Loss on } \\
\text { ignition }\end{array}$ & $\begin{array}{c}\text { Acid } \\
\text { insoluble }\end{array}$ & & \\
\hline $\begin{array}{l}P=3 \\
P-2 \\
P-1\end{array}$ & $\begin{array}{l}\text { Carbonate rock and chert } \\
\text { Carbonate rock lens } \\
\text { Limestone, argillaceous and phosphate } \\
\text { rock- } \\
\text { Phosphate rock, argillaceous, } \\
\text { carbonatic }\end{array}$ & $\begin{array}{l}\text { 3949-RGW } \\
\text { 3948-RGW } \\
\text { 3947-RGW }\end{array}$ & $\begin{array}{l}1.6 \\
(.5) \\
2.4 \\
.3\end{array}$ & $\begin{array}{c}2.6 \\
-- \\
13.8 \\
18.4\end{array}$ & $\begin{array}{l}-- \\
-- \\
--\end{array}$ & $\begin{array}{l}-- \\
-- \\
--\end{array}$ & $\begin{array}{l}-- \\
-- \\
--\end{array}$ & $\begin{array}{l}36.8 \\
-- \\
28.5 \\
22.3\end{array}$ & $\begin{array}{c}142.4 \\
144.8 \\
145.1\end{array}$ & $\begin{array}{l}1,418.67 \\
1,451.79 \\
1,457.31\end{array}$ \\
\hline \multicolumn{11}{|c|}{ Wells formation - top beds only } \\
\hline $\begin{array}{l}\text { Cw-1 } \\
\text { Cw-2 } \\
\text { Cw-3 } \\
\text { Cw-4 } \\
\text { Cw-5 } \\
\text { Cw-6 }\end{array}$ & $\begin{array}{l}\text { Mudstone, carbonatic } \\
\text { Carbonate rock, argillaceous } \\
\text { Carbonate rock, argillaceous } \\
\text { Carbonate rock, argillaceous } \\
\text { Carbonate rock, } \\
\text { Carbonate rock, argillaceous }\end{array}$ & $\begin{array}{l}\text { 3946-RGW } \\
3945-\mathrm{RGW} \\
3944-\mathrm{RGW} \\
3943-\mathrm{RGW} \\
3942-\mathrm{RGW} \\
\text { 3941-RGW }\end{array}$ & $\begin{array}{l}2.2 \\
1.2 \\
1.0 \\
2.2 \\
2.2 \\
1.2\end{array}$ & $\begin{array}{l}2.1 \\
2.8 \\
3.1 \\
-- \\
-- \\
--\end{array}$ & $\begin{array}{l}-- \\
-- \\
-- \\
-- \\
--\end{array}$ & $\begin{array}{l}-- \\
-- \\
-- \\
-- \\
--\end{array}$ & $\begin{array}{l}-- \\
-- \\
-- \\
-- \\
--\end{array}$ & $\begin{array}{l}49.0 \\
36.7 \\
40.7 \\
-- \\
-- \\
--\end{array}$ & $\begin{array}{r}2.2 \\
3.4 \\
4.4 \\
6.6 \\
8.8 \\
10.0\end{array}$ & $\begin{array}{l}-- \\
-- \\
-- \\
-- \\
--\end{array}$ \\
\hline
\end{tabular}


Park City formation measured and phosphatic beds sampled in bulldozer trench and natural outcrop 4 miles east of Morgan in Weber Canyon, sec. 26(?), T. 4 N., R. 3 E., Morgan County, Utah. The section is on the east limb of an anticline. Beds strike N. $40^{\circ}$ E. and dip $20^{\circ}$ E. Section measured by M. A. Warner, T. M. Cheney, R. G. Waring, and R. A. Smart and sampled by T. K. Rigby, Smart, and Warner in June 1951. Samples analyzed by U. S. Bureau of Mines Laboratory, Albany, Oreg.

\begin{tabular}{|c|c|c|c|c|c|c|c|}
\hline \multirow{2}{*}{$\begin{array}{c}\text { Bed } \\
\text { no. }\end{array}$} & \multirow{2}{*}{ Rock description } & \multirow{2}{*}{$\begin{array}{l}\text { Sample } \\
\text { no. }\end{array}$} & \multirow{2}{*}{$\begin{array}{l}\text { Thickness } \\
\text { (feet) }\end{array}$} & \multicolumn{2}{|c|}{$\begin{array}{c}\text { Chemical analyses } \\
\text { (percent) }\end{array}$} & \multirow{2}{*}{$\begin{array}{c}\text { Cumulative } \\
\text { thickness } \\
\text { (feet) }\end{array}$} & \multirow{2}{*}{$\begin{array}{l}\text { Thickness } \mathbf{x} \\
\text { percent } \mathrm{P}_{2} \mathrm{O}_{5} \\
\text { (cumulative) }\end{array}$} \\
\hline & & & & $\mathrm{P}_{2} \mathrm{O}_{5}$ & $\begin{array}{l}\text { Acid } \\
\text { insoluble }\end{array}$ & & \\
\hline \multicolumn{8}{|c|}{ Woodside shale - basal bed only } \\
\hline Tw- 1 & Sandstone & -- & -- & -- & -- & -- & -- \\
\hline \multicolumn{8}{|c|}{ Upper member of Park City formation } \\
\hline $\begin{array}{l}U-17 \\
U-16 \\
U-15 \\
U-14 \\
U-13\end{array}$ & $\begin{array}{l}\text { Carbonate rock } \\
\text { Covered interval } \\
\text { Carbonate rock } \\
\text { Carbonate rock } \\
\text { Covered interval }\end{array}$ & $\begin{array}{l}-- \\
-- \\
-- \\
--\end{array}$ & $\begin{array}{r}4.0 \\
78.0 \\
7.0 \\
4.7 \\
25.6\end{array}$ & $\begin{array}{l}-- \\
-- \\
-- \\
--\end{array}$ & $\begin{array}{l}-- \\
-- \\
-- \\
--\end{array}$ & $\begin{array}{r}4.0 \\
82.0 \\
89.0 \\
93.7 \\
119.3\end{array}$ & $\begin{array}{l}-- \\
-- \\
-- \\
--\end{array}$ \\
\hline $\begin{array}{rr}U-12 \\
U-11 \\
U-10 \\
U- & 9 \\
U- & 8\end{array}$ & $\begin{array}{l}\text { Carbonate rock, sandy } \\
\text { Chert } \\
\text { Carbonate rock, sandy and chert } \\
\text { Sandstone, carbonatic } \\
\text { Carbonate rock, cherty }\end{array}$ & $\begin{array}{l}-- \\
-- \\
-- \\
--\end{array}$ & $\begin{array}{r}15.1 \\
3.5 \\
5.8 \\
6.1 \\
9.6\end{array}$ & $\begin{array}{l}-- \\
-- \\
-- \\
--\end{array}$ & $\begin{array}{l}-- \\
-- \\
-- \\
--\end{array}$ & $\begin{array}{l}134.4 \\
137.9 \\
143.7 \\
149.8 \\
159.4\end{array}$ & $\begin{array}{l}-- \\
-- \\
-- \\
--\end{array}$ \\
\hline $\begin{array}{ll}U- & 7 \\
U- & 6 \\
U- & 5 \\
U- & 4\end{array}$ & $\begin{array}{l}\text { Carbonate rock, cherty } \\
\text { Covered interval } \\
\text { Sandstone } \\
\text { Carbonate rock; fos. col. nos. }\end{array}$ & -- & $\begin{array}{r}8.2 \\
17.4 \\
19.8\end{array}$ & -- & -- & $\begin{array}{l}167.6 \\
185.0 \\
204.8\end{array}$ & -- \\
\hline $\mathrm{U}-\quad 3$ & $\begin{array}{r}12487 \text { and } 12488 \\
\text { Covered interval }\end{array}$ & -- & $\begin{array}{r}28.0 \\
6.0\end{array}$ & -- & -- & $\begin{array}{l}232.8 \\
238.8\end{array}$ & -- \\
\hline $\begin{array}{ll}U- & 2 \\
U- & 1\end{array}$ & $\begin{array}{l}\text { Chert } \\
\text { Carbonate rock, cherty }\end{array}$ & -- & $\begin{array}{l}2.0 \\
1.2\end{array}$ & -- & -- & $\begin{array}{l}240.8 \\
242.0\end{array}$ & -- \\
\hline
\end{tabular}

Phosphatic shale member of Park City formation (?)

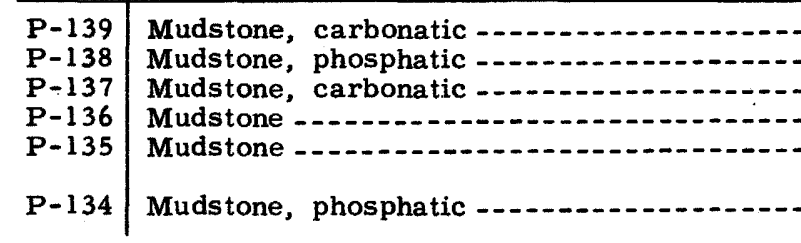

6182-RGW
6181-RGW
6180-RGW
6179-RGW
6178-RGW

7.2
3.2
1.3
1.4
.9
1.8

10.8
2.75
3.65
3.70
13.9

--
53.8
47.6
73.6
66.0
38.0

7.2
10.4
11.7
13.1
14.0
15.8

\footnotetext{
1 Fossil collection made by J. E. Smedley, Paleontology and Stratigraphy Branch, U. S. Geological Survey.
} 
Devils Slide-Continued

\begin{tabular}{|c|c|c|c|c|c|c|c|}
\hline \multirow{2}{*}{$\begin{array}{c}\text { Bed } \\
\text { no. }\end{array}$} & \multirow{2}{*}{ Rock description } & \multirow{2}{*}{$\begin{array}{l}\text { Sample } \\
\text { no. }\end{array}$} & \multirow{2}{*}{$\begin{array}{l}\text { Thickness } \\
\text { (feet) }\end{array}$} & \multicolumn{2}{|c|}{$\begin{array}{c}\text { Chemical analyses } \\
\text { (percent) }\end{array}$} & \multirow{2}{*}{$\begin{array}{c}\text { Cumulative } \\
\text { thickness } \\
\text { (feet) }\end{array}$} & \multirow{2}{*}{$\begin{array}{l}\text { Thickness } \mathrm{x} \\
\text { percent } \mathrm{P}_{2} \mathrm{O}_{5} \\
\text { (cumulative) }\end{array}$} \\
\hline & & & & $\mathrm{P}_{2} \mathrm{O}_{5}$ & $\begin{array}{l}\text { Acid } \\
\text { insoluble }\end{array}$ & & \\
\hline $\begin{array}{l}P-133 \\
P-132 \\
P-131 \\
P-130 \\
P-129\end{array}$ & $\begin{array}{l}\text { Mudstone, phosphatic } \\
\text { Mudstone, phosphatic } \\
\text { Mudstone, carbonatic } \\
\text { Mudstone } \\
\text { Mudstone }\end{array}$ & $\begin{array}{l}\text { 6177- RGW } \\
6176-\text { RGW } \\
\text { 6175- RGW } \\
\text { 6174- RGW } \\
6173-\text { RGW }\end{array}$ & $\begin{array}{l}2.2 \\
1.2 \\
1.9 \\
1.7 \\
1.7\end{array}$ & $\begin{array}{r}10.8 \\
13.8 \\
4.05 \\
4.15 \\
4.10\end{array}$ & $\begin{array}{l}46.5 \\
44.6 \\
40.5 \\
79.0 \\
78.3\end{array}$ & $\begin{array}{l}18.0 \\
19.2 \\
21.1 \\
22.8 \\
24.5\end{array}$ & $\begin{array}{l}95.36 \\
111.92 \\
119.61 \\
126.66 \\
133.64\end{array}$ \\
\hline $\begin{array}{l}P-128 \\
P-127 \\
P-126 \\
P-125 \\
P-124\end{array}$ & $\begin{array}{l}\text { Mudstone } \\
\text { Mudstone } \\
\text { Mudstone } \\
\text { Mudstone, phosphatic } \\
\text { Mudstone }\end{array}$ & $\begin{array}{l}\text { 6172- RGW } \\
6171-\text { RAS } \\
6170-\text { RAS } \\
6169-\text { RAS } \\
6168-\text { RAS }\end{array}$ & $\begin{array}{l}1.4 \\
2.4 \\
4.3 \\
1.9 \\
2.5\end{array}$ & $\begin{array}{r}7.00 \\
3.95 \\
2.65 \\
13.5 \\
2.45\end{array}$ & $\begin{array}{l}60.6 \\
65.8 \\
71.6 \\
49.8 \\
79.5\end{array}$ & $\begin{array}{l}25.9 \\
28.3 \\
32.6 \\
34.5 \\
37.0\end{array}$ & $\begin{array}{l}143.44 \\
152.92 \\
164.31 \\
189.96 \\
196.08\end{array}$ \\
\hline $\begin{array}{l}P-123 \\
P-122 \\
P-121 \\
P-120 \\
P-119\end{array}$ & $\begin{array}{l}\text { Phosphate rock, argillaceous } \\
\text { Carbonate rock, argillaceous } \\
\text { Mudstone } \\
\text { Mudstone, carbonatic } \\
\text { Mudstone, phosphatic }\end{array}$ & $\begin{array}{l}\text { 6167- RAS } \\
6166-\text { RAS } \\
6165-\text { RAS } \\
6164-\text { RAS } \\
6163-\text { RAS }\end{array}$ & $\begin{array}{r}1.5 \\
1.1 \\
3.1 \\
.7 \\
1.3\end{array}$ & $\begin{array}{l}19.9 \\
1.10 \\
6.05 \\
1.10 \\
10.6\end{array}$ & $\begin{array}{l}41.3 \\
29.6 \\
73.1 \\
45.7 \\
61.6\end{array}$ & $\begin{array}{l}38.5 \\
39.6 \\
42.7 \\
43.4 \\
44.7\end{array}$ & $\begin{array}{l}225.94 \\
227.14 \\
245.90 \\
246.67 \\
260.45\end{array}$ \\
\hline $\begin{array}{l}P-118 \\
P-117 \\
P-116 \\
P-115 \\
P-114\end{array}$ & $\begin{array}{l}\text { Mudstone, carbonatic } \\
\text { Mudstone, phosphatic } \\
\text { Carbonate rock } \\
\text { Mudstone, phosphatic } \\
\text { Carbonate rock, argillaceous }\end{array}$ & $\begin{array}{l}\text { 6162- RAS } \\
6161-\text { RAS } \\
6160-\text { RAS } \\
\text { 6159- RAS } \\
\text { 6158- TMC }\end{array}$ & $\begin{array}{l}2.2 \\
1.1 \\
1.0 \\
2.4 \\
1.5\end{array}$ & $\begin{aligned} 1.90 \\
10.1 \\
1.30 \\
10.2 \\
1.25\end{aligned}$ & $\begin{array}{l}55.3 \\
59.8 \\
15.2 \\
63.0 \\
32.4\end{array}$ & $\begin{array}{l}46.9 \\
48.0 \\
49.0 \\
51.4 \\
52.9\end{array}$ & $\begin{array}{l}264.63 \\
275.74 \\
277.04 \\
301.52 \\
303.40\end{array}$ \\
\hline $\begin{array}{l}P-113 \\
P-11 \\
P-11 \\
P-110 \\
P-109\end{array}$ & $\begin{array}{l}\text { Mudstone, phosphatic } \\
\text { Mudstone, carbonatic } \\
\text { Carbonate rock } \\
\text { Mudstone } \\
\text { Phosphate rock, argillaceous, phosphatic } \\
\text { mudstone, mudstone and phosphate rock- }\end{array}$ & $\begin{array}{l}\text { 6157- TMC } \\
6156-\text { TMC } \\
6155-\text { TMC } \\
6154-\text { TMC } \\
6153-\text { TMC }\end{array}$ & $\begin{array}{r}1.8 \\
.7 \\
.8 \\
1.2 \\
.9\end{array}$ & $\begin{array}{c}11.4 \\
4.90 \\
1.55 \\
4.97 \\
19.4\end{array}$ & $\begin{array}{l}56.8 \\
53.6 \\
18.5 \\
68.7 \\
29.5\end{array}$ & $\begin{array}{l}54.7 \\
55.4 \\
56.2 \\
57.4 \\
58.3\end{array}$ & $\begin{array}{l}323.92 \\
327.34 \\
328.58 \\
334.55 \\
352.01\end{array}$ \\
\hline $\begin{array}{l}P-108 \\
P-107 \\
P-106 \\
P-105 \\
P-104\end{array}$ & $\begin{array}{l}\text { Mudstone, carbonatic, phosphate rock } \\
\text { and muds tone } \\
\text { Mudstone, calcareous } \\
\text { Mudstone, calcareous, phosphatic } \\
\text { Phosphate rock, argillaceous and } \\
\text { phosphatic mudstone } \\
\text { Carbonate rock, argillaceous }\end{array}$ & $\begin{array}{l}\text { 6152-TMC } \\
\text { 6151-TMC } \\
\text { 6150- TMC } \\
\text { 6149- TMC } \\
\text { 6148- TMC }\end{array}$ & $\begin{array}{l}1.4 \\
3.4 \\
1.8 \\
1.8 \\
2.1\end{array}$ & $\begin{array}{r}7.20 \\
2.45 \\
6.05 \\
23.5 \\
.50\end{array}$ & $\begin{array}{l}51.5 \\
43.7 \\
64.4 \\
28.2 \\
25.0\end{array}$ & $\begin{array}{l}59.7 \\
63.1 \\
64.9 \\
66.7 \\
68.8\end{array}$ & $\begin{array}{l}362.09 \\
370.42 \\
381.31 \\
423.61 \\
424.66\end{array}$ \\
\hline $\begin{array}{l}P-103 \\
P-102 \\
P-101 \\
P-100\end{array}$ & $\begin{array}{l}\text { Phosphate rock and phosphatic mudstone } \\
\text { Mudstone and calcareous mudstone } \\
\text { Phosphate rock, argillaceous } \\
\text { Mudstone, carbonatic and phosphatic } \\
\text { mudstone. }\end{array}$ & $\begin{array}{l}\text { 6147- TMC } \\
6146-\text { TMC } \\
6145-\text { TMC } \\
6144-\text { TMC }\end{array}$ & $\begin{array}{r}.8 \\
1.7 \\
.5 \\
1.0\end{array}$ & $\begin{array}{l}17.4 \\
3.30 \\
18.8 \\
4.80\end{array}$ & $\begin{array}{l}32.4 \\
40.2 \\
25.3 \\
56.2\end{array}$ & $\begin{array}{l}69.6 \\
71.3 \\
71.8 \\
72.8\end{array}$ & $\begin{array}{l}438.58 \\
444.19 \\
453.59 \\
458.39\end{array}$ \\
\hline
\end{tabular}




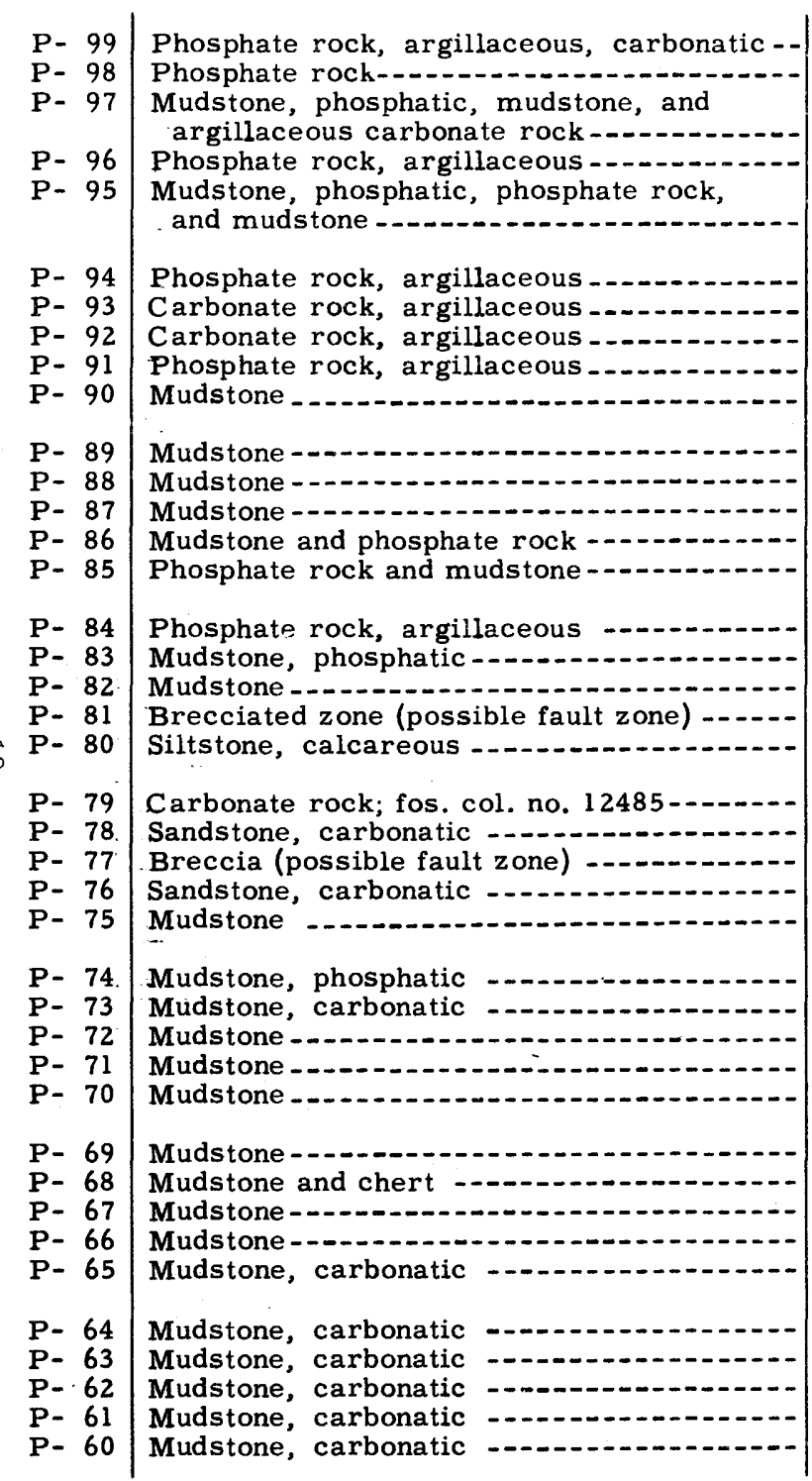

\begin{tabular}{|c|}
\hline $\begin{array}{l}\text { 6143-TMC } \\
6142-\text { TMC }\end{array}$ \\
\hline $\begin{array}{l}\text { 6141-TMC } \\
\text { 6140- TIMC }\end{array}$ \\
\hline $6139-$ TMC \\
\hline $\begin{array}{l}6138-\mathrm{TMC} \\
6137-\mathrm{TMC} \\
6136-\mathrm{TMC} \\
6135-\mathrm{TMC} \\
6134-\mathrm{TMC}\end{array}$ \\
\hline $\begin{array}{l}\text { 6133- TMC } \\
6130-\text { TMC } \\
6129-\text { TMC } \\
6128-\text { TMC } \\
6127-\text { TMC }\end{array}$ \\
\hline $\begin{array}{l}\text { 6126- TMC } \\
6125-\text { TMC } \\
6124-\text { TMC }\end{array}$ \\
\hline-- \\
\hline-- \\
\hline-- \\
\hline-- \\
\hline-- \\
\hline $\begin{array}{c}\text { 6132-MAW } \\
\text { 6131-MAW } \\
-- \\
-- \\
--\end{array}$ \\
\hline- \\
\hline-- \\
\hline \\
\hline 6123-MAW \\
\hline $\begin{array}{l}\text { 6122-MAW } \\
\text { 6121-MAW } \\
\text { 6120-MAW } \\
6119-\text { MAW } \\
6118-\text { MAW }\end{array}$ \\
\hline
\end{tabular}

\begin{tabular}{|c|c|}
\hline $\begin{array}{l}15.0 \\
23.4\end{array}$ & $\begin{array}{l}23.2 \\
21.5\end{array}$ \\
\hline $\begin{array}{r}2.70 \\
22.4\end{array}$ & $\begin{array}{l}17.4 \\
28.2\end{array}$ \\
\hline 15.0 & 45.0 \\
\hline $\begin{array}{r}22.1 \\
.45 \\
2.15 \\
25.8 \\
.75\end{array}$ & $\begin{array}{l}31.3 \\
33.7 \\
35.5 \\
27.2 \\
86.8\end{array}$ \\
\hline $\begin{array}{r}1.05 \\
.75 \\
1.15 \\
13.4 \\
20.8\end{array}$ & $\begin{array}{l}85.8 \\
81.8 \\
83.7 \\
52.4 \\
35.6\end{array}$ \\
\hline $\begin{array}{r}22.8 \\
17.5 \\
6.20\end{array}$ & $\begin{array}{l}26.4 \\
46.0 \\
75.2\end{array}$ \\
\hline-- & -- \\
\hline-- & \\
\hline-- & -- \\
\hline-- & -- \\
\hline$\cdots$ & - \\
\hline-- & -- \\
\hline $\begin{array}{r}10.0 \\
4.65\end{array}$ & $\begin{array}{l}53.1 \\
40.1\end{array}$ \\
\hline- & -- \\
\hline-- & - \\
\hline-- & -- \\
\hline-- & - \\
\hline-- & -- \\
\hline- & - \\
\hline 2.25 & 58.1 \\
\hline $\begin{array}{l}2.00 \\
8.85 \\
3.45 \\
5.70 \\
4.70\end{array}$ & $\begin{array}{l}52.2 \\
46.9 \\
52.1 \\
50.8 \\
38.2\end{array}$ \\
\hline
\end{tabular}

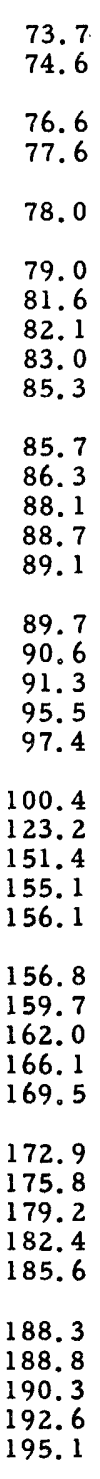

471.89 492.95

498.35 520.75

526. 75

548.85 550.02 551.09 574. 31

576.46

576.46
576.91 576.91
578.98 587.02 595. 34

609.02 624.77 $\frac{629.11}{--}$

* Cumulative data incomplete because of missing information. Cumulatives start from zero after interruption. 
Devils Slide - Continued

\begin{tabular}{|c|c|c|c|c|c|c|c|}
\hline \multirow{2}{*}{$\begin{array}{c}\text { Bed } \\
\text { no. }\end{array}$} & \multirow{2}{*}{ Rock description } & \multirow{2}{*}{$\begin{array}{l}\text { Sample } \\
\text { no. }\end{array}$} & \multirow{2}{*}{$\begin{array}{l}\text { Thickness } \\
\text { (feet) }\end{array}$} & \multicolumn{2}{|c|}{$\begin{array}{c}\text { Chemical analyses } \\
\text { (percent) }\end{array}$} & \multirow{2}{*}{$\begin{array}{l}\text { Cumulative } \\
\text { thickness } \\
\text { (feet) }\end{array}$} & \multirow{2}{*}{$\begin{array}{l}\text { Thickness } \mathbf{x} \\
\text { percent } \mathrm{P}_{2} \mathrm{O}_{5} \\
\text { (cumulative) }\end{array}$} \\
\hline & & & & $\mathrm{P}_{2} \mathrm{O}_{5}$ & $\begin{array}{l}\text { Acid } \\
\text { insoluble }\end{array}$ & & \\
\hline $\begin{array}{l}P-59 \\
P-58 \\
P-57 \\
P-56 \\
P-55\end{array}$ & $\begin{array}{l}\text { Mudstone, carbonatic } \\
\text { Mudstone, carbonatic } \\
\text { Mudstone, carbonatic } \\
\text { Phosphate rock, carbonatic, argillaceous } \\
\text { Mudstone }\end{array}$ & $\begin{array}{l}\text { 6117-MAW } \\
6116-\text { MAW } \\
6115-\text { MAW } \\
6114-\text { MAW } \\
--\end{array}$ & $\begin{array}{l}1.5 \\
1.5 \\
1.4 \\
.35 \\
2.7\end{array}$ & $\begin{array}{l}3.50 \\
1.75 \\
4.00 \\
17.2 \\
--\end{array}$ & $\begin{array}{l}42.2 \\
40.2 \\
45.7 \\
22.6 \\
-.\end{array}$ & $\begin{array}{l}196.6 \\
198.1 \\
199.5 \\
199.85 \\
202.55\end{array}$ & $\begin{array}{l}52.31 \\
54.94 \\
60.54 \\
66.56 \\
--\end{array}$ \\
\hline $\begin{array}{l}P-54 \\
P-53 \\
P-52 \\
P-51 \\
P-50\end{array}$ & $\begin{array}{l}\text { Mudstone } \\
\text { Mudstone } \\
\text { Mudstone, cherty and mudstone cherty } \\
\text { Mudstone, cherty }\end{array}$ & $\begin{array}{l}-- \\
-- \\
-- \\
--\end{array}$ & $\begin{array}{l}3.0 \\
3.6 \\
1.3 \\
1.4 \\
2.8\end{array}$ & $\begin{array}{l}-- \\
-- \\
-- \\
--\end{array}$ & $\begin{array}{l}-- \\
-- \\
-- \\
--\end{array}$ & $\begin{array}{l}205.55 \\
209.15 \\
210.45 \\
211.85 \\
214.65\end{array}$ & $\begin{array}{l}-- \\
-- \\
-- \\
--\end{array}$ \\
\hline $\begin{array}{l}P-49 \\
P-48 \\
P-47 \\
P-46 \\
P-45\end{array}$ & $\begin{array}{l}\text { Mudstone } \\
\text { Mudstone, cherty } \\
\text { Mudstone } \\
\text { Mudstone } \\
\text { Mudstone, carbonatic }\end{array}$ & $\begin{array}{l}-- \\
-- \\
-- \\
-- \\
6113-\text { MAW }\end{array}$ & $\begin{array}{r}1.9 \\
.9 \\
2.3 \\
.9 \\
.8\end{array}$ & $\begin{array}{l}-- \\
-- \\
-- \\
-- \\
1.30\end{array}$ & $\begin{array}{l}-- \\
-- \\
-- \\
-- \\
54.8\end{array}$ & $\begin{array}{l}216.55 \\
217.45 \\
219.75 \\
220.65 \\
221.45\end{array}$ & $\begin{array}{l}-- \\
-- \\
-- \\
-- \\
* 1.04\end{array}$ \\
\hline $\begin{array}{l}P-44 \\
P-43 \\
P-42 \\
P-41 \\
P-40\end{array}$ & $\begin{array}{l}\text { Mudstone, carbonatic } \\
\text { Mudstone } \\
\text { Mudstone } \\
\text { Mudstone } \\
\text { Mudstone, carbonatic }\end{array}$ & $\begin{array}{l}\text { 6112-MAW } \\
6111-\text { MAW } \\
6110-\text { MAW } \\
6109-\text { MAW } \\
6108-\text { MAW }\end{array}$ & $\begin{array}{r}3.0 \\
.9 \\
1.8 \\
1.3 \\
2.2\end{array}$ & $\begin{array}{l}.04 \\
1.35 \\
3.15 \\
4.90 \\
1.15\end{array}$ & $\begin{array}{l}47.4 \\
83.7 \\
78.5 \\
73.2 \\
55.0\end{array}$ & $\begin{array}{l}224.45 \\
225.35 \\
227.15 \\
228.45 \\
230.65\end{array}$ & $\begin{array}{r}1.16 \\
2.38 \\
8.04 \\
14.42 \\
16.94\end{array}$ \\
\hline $\begin{array}{l}P-39 \\
P-38 \\
P-37 \\
P-36 \\
P-35\end{array}$ & $\begin{array}{l}\text { Mudstone, carbonatic } \\
\text { Mudstone, carbonatic } \\
\text { Carbonate rock } \\
\text { Mudstone } \\
\text { Mudstone }\end{array}$ & $\begin{array}{l}\text { 6107-MAW } \\
\text { 6106-MAW } \\
\text { 6105-MAW } \\
\text { 6104-MAW } \\
\text { 6103-MAW }\end{array}$ & $\begin{array}{l}5.5 \\
3.7 \\
1.1 \\
1.6 \\
1.5\end{array}$ & $\begin{array}{r}.40 \\
.50 \\
.55 \\
1.25 \\
6.60\end{array}$ & $\begin{array}{r}59.3 \\
63.6 \\
7.3 \\
72.5 \\
66.5\end{array}$ & $\begin{array}{l}236.15 \\
239.85 \\
240.95 \\
242.55 \\
244.05\end{array}$ & $\begin{array}{l}19.14 \\
21.00 \\
21.60 \\
23.60 \\
33.50\end{array}$ \\
\hline $\begin{array}{l}P-34 \\
P-33 \\
P-32 \\
P-31 \\
P-30\end{array}$ & $\begin{array}{l}\text { Mudstone } \\
\text { Mudstone } \\
\text { Mudstone } \\
\text { Mudstone, carbonatic } \\
\text { Mudstone, carbonatic }\end{array}$ & $\begin{array}{l}6102-\text { MAW } \\
6101-\text { MAW } \\
6100-\text { MAW } \\
6099-\text { MAW } \\
6098-\text { MAW }\end{array}$ & $\begin{array}{l}2.6 \\
2.9 \\
3.1 \\
3.2 \\
1.1\end{array}$ & $\begin{array}{l}.70 \\
1.20 \\
6.60 \\
4.85 \\
2.25\end{array}$ & $\begin{array}{l}81.6 \\
82.8 \\
67.2 \\
55.4 \\
65.2\end{array}$ & $\begin{array}{l}246.65 \\
249.55 \\
252.65 \\
255.85 \\
256.95\end{array}$ & $\begin{array}{l}35.32 \\
38.80 \\
50.26 \\
74.78 \\
77.26\end{array}$ \\
\hline $\begin{array}{l}P-29 \\
P-28 \\
P-27\end{array}$ & $\begin{array}{l}\text { Mudstone } \\
\text { Limestone; fos. col. no. } 12484 .\end{array}$ & $\begin{array}{l}\text { 6097-MAW } \\
6096-\text { MAW }\end{array}$ & $\begin{array}{l}1.2 \\
4.1\end{array}$ & $\begin{array}{r}3.30 \\
.70\end{array}$ & $\begin{array}{l}74.7 \\
16.5\end{array}$ & $\begin{array}{l}258.15 \\
262.25\end{array}$ & $\begin{array}{l}81.22 \\
84.08\end{array}$ \\
\hline $\begin{array}{l}P-26 \\
P-25\end{array}$ & $\begin{array}{l}12484 \\
\text { Mudstone, carbonatic } \\
\text { Mudstone, carbonatic }\end{array}$ & $\begin{array}{l}\text { 6095-MAW } \\
6094-\mathrm{MAW} \\
6093-\mathrm{MAW}\end{array}$ & $\begin{array}{l}1.9 \\
2.0 \\
1.4\end{array}$ & $\begin{array}{r}.80 \\
5.45 \\
5.35\end{array}$ & $\begin{array}{l}27.3 \\
54.3 \\
46.9\end{array}$ & $\begin{array}{l}264.15 \\
266.15 \\
267.55\end{array}$ & $\begin{array}{r}85.60 \\
96.51 \\
104.00\end{array}$ \\
\hline$P-24$ & Carbonate rock and phosphate rock & 6092-MAW & 1.0 & 8.00 & 12.8 & 268.55 & 112.00 \\
\hline
\end{tabular}


\begin{tabular}{l|l} 
P-23 & Mudstone and phosphatic mudstone - -0.1
\end{tabular} P-22 Carbonate rock, phosphatic -..--

P-21 Phosphate rock, argillaceous -.............

Pudstone, carbonatic--and

Mudstone, phosphatic

P-18 Carbonate rock, phosphatic, argillaceous---

Mudstone, carbonatic, phosphatic -

Mudstone, phosphatic - carbonatic, argillaceous -

Carbonate rock

P-13 Phosphate rock, carbonatic, argillaceous --

Carbonate rock, phosphatic, argillace
Brecciated muds tone containing much

calcite and chert

P-10 Carbonate rock, argillaceous; fos. col.

P- 9 no. 12483 -

Mudstone, phosphatic

P- 8

P- 7 Carbonate rock, argillaceous -

P- 6 Carbonate rock; fos. col. no. 12482

P- 5 Carbonate rock; fos. col. no. 12482

ज $P$

$P-3$
$P-2$

Mudstone -

Mudstone, carbonatic -

Carbonate rock, argillaceor

aceous

Mudstone, phosphatic, and argillaceous

phosphate rock
6091-MAW 6090-MAW 6089-MAW 6088-MAW 6087-MAW

6086-MAW 6085-MAW 6084-MAW 6083-MAW 6082-MAW

6081-MAW 6080-MAW

--

6079-MAW 6078-MAW

6077-MAW 6076-MAW 6075-MAW 6074-MAW $--$

$--$

6185-RAS

0
1.
2.
1.
1.
2.
1.
1.
1.
1.
2.
3.

\begin{tabular}{c|c}
11.2 & 43.1 \\
14.2 & 10.6 \\
16.3 & 32.9 \\
4.70 & 52.1 \\
14.7 & 37.4 \\
9.25 & 23.4 \\
8.10 & 40.8 \\
13.5 & 47.2 \\
14.6 & 24.7 \\
2.60 & 7.7 \\
18.1 & 22.6 \\
10.4 & 23.5 \\
-- &.- \\
1.35 & 33.3 \\
2.15 & 58.9 \\
10.5 & 57.9 \\
3.95 & 26.7 \\
4.75 & 14.5 \\
7.50 & 12.5 \\
-- & -- \\
-- & -- \\
-- & -- \\
11.6 & 45.9
\end{tabular}

269.25

270.15

271.15

272.95

275.25

276. 15

278.05

280.15

280.95

281.95

283.15

284.05

286.25

288.25

289.65

290.65

291.85

292.55

293. 75

295.15

297.45

301.35

1. 7

11.6

45.9

303.05

119.84

132.62

148.92

157.38

191.18

199.51

214.90

243.25

254.93

257.53

279.25

288.61

-.

*2. 70

5.71

16. 21

20.95

24.28

* 33.28

--

$--$

$-$

-

Lower member of Park City formation (?)

\begin{tabular}{|c|c|}
\hline & fragments of cherty \\
\hline & 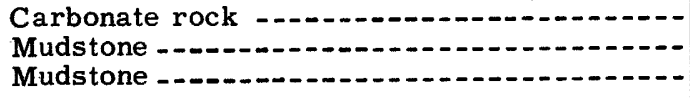 \\
\hline $\begin{array}{l}5 \\
4\end{array}$ & $\begin{array}{l}\text { Mud } \\
\text { Mud } \\
\text { Mud }\end{array}$ \\
\hline $\mathbf{L}-$ & $\begin{array}{l}\text { uartzite, argill } \\
\text { arbonate rock, }\end{array}$ \\
\hline
\end{tabular}

--
--
--
--
--
--
--
--
- RAS
--
--

3.3

\begin{tabular}{c|c}
-- & - \\
-- & -- \\
-- & -- \\
-- & -- \\
-- & -- \\
-- & -- \\
-- & -- \\
-- & -- \\
-- & -- \\
-- & -- \\
15.9 & 42.3 \\
-- & -- \\
-- & --
\end{tabular}

3.3
14.3
17.8
19.7
20.4
22.6
24.1
26.0
27.6
30.4
31.2
33.0
35.0

* Cumulative data incomplete because of missing information. Cumulatives start from zero after interruption.

* Note incompleteness of cumulative data. 
Devils Slide-Continued

\begin{tabular}{|c|c|c|c|c|c|c|c|}
\hline \multirow{2}{*}{$\begin{array}{c}\text { Bed } \\
\text { no. }\end{array}$} & \multirow{2}{*}{ Rock description } & \multirow{2}{*}{$\begin{array}{l}\text { Sample } \\
\text { no. }\end{array}$} & \multirow{2}{*}{$\begin{array}{c}\text { Thickness } \\
\text { (feet) }\end{array}$} & \multicolumn{2}{|c|}{$\begin{array}{l}\text { Chemical analyses } \\
\text { (percent) }\end{array}$} & \multirow{2}{*}{$\begin{array}{l}\text { Cumulative } \\
\text { thickness } \\
\text { (feet) }\end{array}$} & \multirow{2}{*}{$\begin{array}{l}\text { Thickness } \mathrm{x} \\
\text { percent } \mathrm{P}_{2} \mathrm{O}_{5} \\
\text { (cumulative) }\end{array}$} \\
\hline & & & & $\mathrm{P}_{2} \mathrm{O}_{5}$ & $\begin{array}{l}\text { Acid } \\
\text { insoluble }\end{array}$ & & \\
\hline $\begin{array}{l}L-28 \\
L-27 \\
L-26 \\
L-25 \\
L-24\end{array}$ & $\begin{array}{l}\text { Carbonate rock, cherty } \\
\text { Carbonate rock } \\
\text { Mudstone, carbonatic } \\
\text { Mudstone and phosphate rock } \\
\text { Mudstone, carbonatic }\end{array}$ & $\begin{array}{c}-- \\
-- \\
-- \\
6183-\text { RAS } \\
--\end{array}$ & $\begin{array}{c}3.6 \\
2.5 \\
2.9 \\
(1.1) \\
(2.2)\end{array}$ & $\begin{array}{l}-- \\
-- \\
16.4 \\
--\end{array}$ & $\begin{array}{l}-- \\
-- \\
-- \\
47.5 \\
--\end{array}$ & $\begin{array}{l}38.6 \\
41.1 \\
44.0 \\
-- \\
--\end{array}$ & $\begin{array}{l}-- \\
-- \\
-- \\
--\end{array}$ \\
\hline$L-23$ & $\begin{array}{l}\text { Mudstone, carbonatic; fos. col. no. } \\
12481\end{array}$ & -- & $(2.3)$ & -- & -- & $\ldots$ & -- \\
\hline $\begin{array}{l}L-22 \\
L-21 \\
L-20 \\
L-19 .\end{array}$ & $\begin{array}{l}\text { Mudstone } \\
\text { Mudstone, carbonatic } \\
\text { Mudstone; fos. col. no. } 12480 \\
\text { mudstone, carbonatic, chert, and }\end{array}$ & $\begin{array}{l}-- \\
-- \\
-- \\
--\end{array}$ & $\begin{array}{r}(.8) \\
(1.7) \\
(.8) \\
(.6)\end{array}$ & $\begin{array}{l}-- \\
-- \\
-- \\
--\end{array}$ & $\begin{array}{l}-- \\
-- \\
-- \\
-\infty\end{array}$ & $\begin{array}{l}-- \\
-- \\
-- \\
--\end{array}$ & $\begin{array}{l}-- \\
-- \\
-- \\
--\end{array}$ \\
\hline $\begin{array}{l}L-18 \\
L-17\end{array}$ & $\begin{array}{l}\text { Carbonate rock } \\
\text { Mudstone, carbonatic; fos. col. no. }\end{array}$ & -- & $\begin{array}{r}(2.9) \\
(.9)\end{array}$ & -- & -- & - & -- \\
\hline $\begin{array}{l}L-16 \\
L-15 \\
L-14\end{array}$ & $\begin{array}{l}\text { Mudstone } \\
\text { Mudstone } \\
\text { Mudstone, carbonatic }\end{array}$ & $\begin{array}{ll}-- \\
-- \\
--\end{array}$ & $\begin{array}{r}(.8) \\
(3.9) \\
(2.3)\end{array}$ & $\begin{array}{ll}-- \\
-- \\
--\end{array}$ & $\begin{array}{ll}-- \\
--\end{array}$ & -- & $\begin{array}{l}-- \\
-- \\
--\end{array}$ \\
\hline$L-13$ & $\begin{array}{l}\text { Mudstone, carbonatic; fos. col. no. } \\
12478\end{array}$ & -- & $(2.5)$ & -- & -- & -- & -- \\
\hline $\begin{array}{l}L-12 \\
L-11 \\
L-10\end{array}$ & $\begin{array}{l}\text { Carbonate rock } \\
\text { Mudstone } \\
\text { Carbonate rock, argillaceous }\end{array}$ & -- & $\begin{array}{r}(2.4) \\
(.9) \\
(3.3)\end{array}$ & -- & -- & $\ddot{--}$ & -- \\
\hline & $\begin{array}{l}\text { Section possibly faulted, remainder } \\
\text { measured on north side of canyon. Bed } \\
\mathrm{L}-25 \text { correlated with beds } \mathrm{L}-6,7 \text {, and } 8 \text {; } \\
\text { beds } \mathrm{L}-10 \text { through } \mathrm{L}-24 \text { represent upper } \\
\text { part of } \mathrm{L}-5 \text {. Beds below } \mathrm{L}-6 \text { poorly } \\
\text { exposed and not described in detail. }\end{array}$ & & & & & & \\
\hline L- 9 & Mudstone - & -- & $(.7)$ & -- & -- & -- & -- \\
\hline L- 8 & Mudstone, phosphatic & 6188-RGW & 1.2 & 14.4 & 44.3 & 45.2 & -- \\
\hline $\begin{array}{l}L-7 \\
L-6\end{array}$ & $\begin{array}{l}\text { Mudstone } \\
\text { Carbonate rock, argillaceous }\end{array}$ & $\begin{array}{l}\text { 6187-RGW } \\
\text { 6186-RGW }\end{array}$ & .5 & $\begin{array}{l}6.10 \\
4.15\end{array}$ & $\begin{array}{l}68.3 \\
33.4\end{array}$ & $\begin{array}{l}45.7 \\
46.4\end{array}$ & -- \\
\hline L- 5 & Muds tone & - & 65.0 & -- & $\therefore$ & 111.4 & -- \\
\hline L- 4 & Mudstone & -- & 77.0 & -- & - & 188.4 & - \\
\hline & $\begin{array}{l}\text { Carbonate rock, chert, and cherty } \\
\text { carbonate rock }\end{array}$ & -- & 27.0 & - & -- & 215.4 & -- \\
\hline$L-2$ & 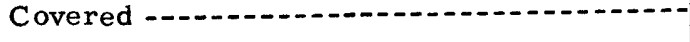 & -- & 97.0 & -- & -- & 312.4 & -- \\
\hline
\end{tabular}




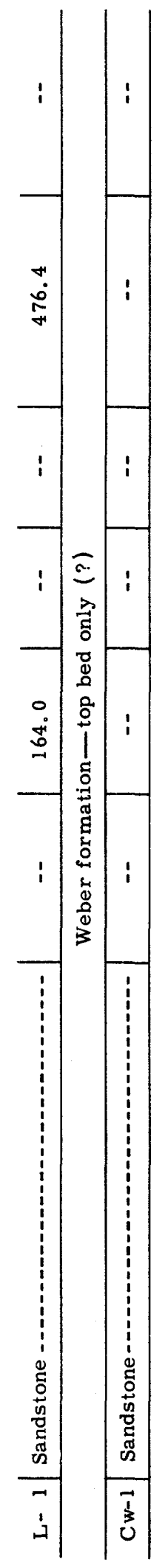


Park City formation measured and sampled at natural exposure on the north flank of the Uinta Mountains axis in Franson Canyon, sec. 14, T. 1 S., R. 6 E., Summit County, Utah. Beds strike N. $80^{\circ}$ E. and dip $30^{\circ}$ N. Section measured and sampled by T. M. Cheney,

R. A. Smart, M. A. Warner, and R. G. Waring in June 1951. Samples analyzed by Trace Elements Section laboratory, Denver,

Colo.

\begin{tabular}{|c|c|c|c|c|c|c|c|}
\hline \multirow{2}{*}{$\begin{array}{c}\text { Bed } \\
\text { no. }\end{array}$} & \multirow{2}{*}{ Rock description } & \multirow{2}{*}{$\begin{array}{c}\text { Sample } \\
\text { no. }\end{array}$} & \multirow{2}{*}{$\begin{array}{l}\text { Thickness } \\
\text { (feet) }\end{array}$} & \multicolumn{2}{|c|}{$\begin{array}{c}\text { Chemical analyses } \\
\text { (percent) }\end{array}$} & \multirow{2}{*}{$\begin{array}{c}\text { Cumulative } \\
\text { thickness } \\
\text { (feet) }\end{array}$} & \multirow{2}{*}{$\begin{array}{l}\text { Thickness } \mathrm{x} \\
\text { percent } \mathrm{P}_{2} \mathrm{O}_{5} \\
\text { (cumulative) }\end{array}$} \\
\hline & & & & $\mathrm{P}_{2} \mathrm{O}_{5}$ & $\begin{array}{c}\text { Acid } \\
\text { insoluble }\end{array}$ & & \\
\hline
\end{tabular}

Upper member of Park City formation - top not exposed

Woodside-Park City contact covered approximate thickness of covered interval

U-70 Carbonate rock; fos. col. no. $12500^{1} \ldots . . .-$

U-69 Carbonate rock, argillaceous; fos. col no. 12500 rock, argillaceous; fos. col. Carbonate rock; fos. col. no. 12500 -....Carbonate rock, fos, col no 12500 -.--Corbonate rock, argillaceous; fos. col. no. 12500

Carbonate rock; fos. col. no. 12500 ....--

Siltstone, carbonatic; fos. col. no. 12500 -.-

U-63 Siltstone, carbonatic; fos. col. no. $12500 \ldots$

$\mathrm{U}-61$

Siltstone, carbonatic

$-61$

$\mathrm{U}-60$

$\mathrm{U}-59$

$\mathrm{U}-58$

$\mathrm{U}-57$

$\mathrm{U}-56$

$\mathrm{U}-55$

$\mathrm{U}-54$

$\mathrm{U}-54$

$\mathrm{U}-53$

$\mathrm{U}-52$

$U-50$

$\mathrm{U}-49$

$\mathrm{U}-48$

$\mathrm{U}-47$

$\mathrm{U}-46$

Silts tone, carbonatic

Siltstone, carbonatic; fos. col. no. 12499 -.-

Carbonate rock-

Sandstone, carbonatic

Carbonate rock

Mudstone, carbonatic -

Mudstone, carbonatic .

Mudstone, carbonatic -

Carbonate rock, argillaceous -

Silts tone -

Siltstone, carbonatic -

Carbonate rock, argillaceous -

Mudstone, carbonatic -

\begin{tabular}{|c|} 
\\
-- \\
-- \\
-- \\
-- \\
-- \\
-- \\
-- \\
-- \\
-- \\
-- \\
-- \\
-- \\
$6320-$ \\
-- \\
-- \\
-- \\
-- \\
-- \\
-- \\
-- \\
-- \\
-- \\
-
\end{tabular}

15.0

2.8

3.2

3. 1

3.2

2. 7

1.9

2. 5

1. 7

1. 2

1. 9

2. 0

.4
4

2. 8

4. 4

1.3

1.8

1. 2

3. 1

7.2

12.4

1.2

1.2
.7

\begin{tabular}{c|c|} 
& \\
-- & -- \\
-- & -- \\
-- & -- \\
-- & -- \\
-- & -- \\
-- & -- \\
-- & -- \\
-- & -- \\
-- & -- \\
-- & -- \\
-- & -- \\
-- & -- \\
-- & -- \\
-- & -- \\
6.9 & 31.3 \\
-- & -- \\
-- & -- \\
-- & -- \\
-- & -- \\
-- & -- \\
-- & -- \\
-- & -- \\
-- & -- \\
-- & -- \\
-- & -- \\
-- & -- \\
\hline
\end{tabular}

15.0

17.8

21.0

24.1

27.3

30.0

31.9

34.4

36.1

37.3

40.2

42. 1

44.2

46. 2

46.6

51.3

54.1

58.5

59.8

61.6

62.8

65.9

73.1

85. 5

86.7

87.4
--
--
--
--
--
--
-
--
--
--
--
--
-
--
--
--
--
--
--
-
- 


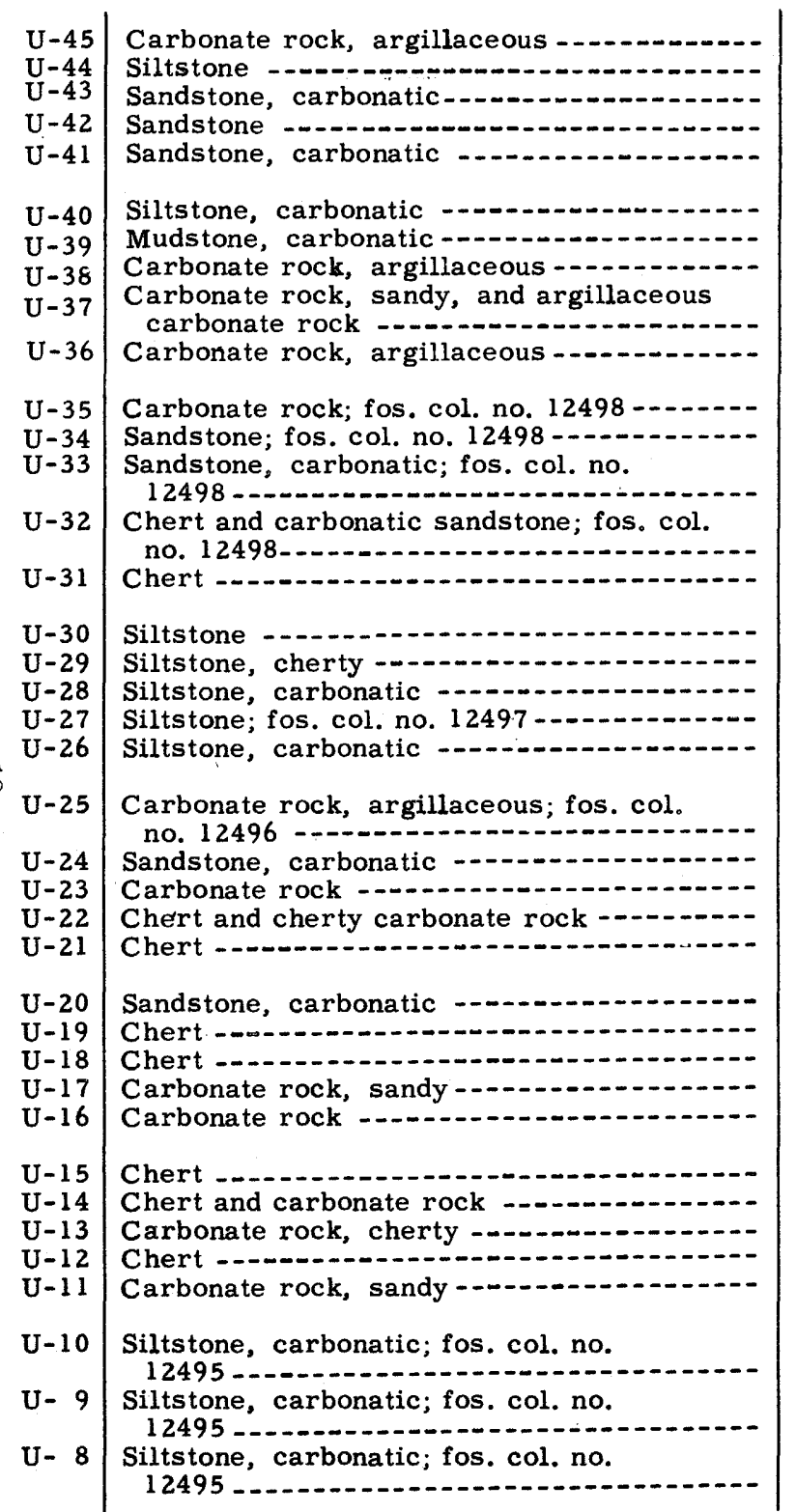

\begin{tabular}{|c|c|}
\hline - & $\begin{array}{r}8.1 \\
3.9 \\
2.2 \\
8.7 \\
12.9\end{array}$ \\
\hline $\begin{array}{l}\text { MAW } \\
\text { MAW }\end{array}$ & $\begin{array}{l}5.3 \\
2.2 \\
1.5\end{array}$ \\
\hline . & $\begin{array}{l}3.4 \\
5.6\end{array}$ \\
\hline . & $\begin{array}{l}3.0 \\
2.3\end{array}$ \\
\hline & 2.0 \\
\hline & $\begin{array}{l}1.9 \\
1.9\end{array}$ \\
\hline 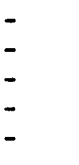 & $\begin{array}{l}1.7 \\
1.9 \\
1.5 \\
3.9 \\
1.5\end{array}$ \\
\hline $\begin{array}{l}- \\
\overline{-} \\
-\end{array}$ & $\begin{array}{r}1.9 \\
1.0 \\
.5 \\
1.0 \\
1.0\end{array}$ \\
\hline$\overline{-}$ & $\begin{array}{l}1.8 \\
1.8 \\
2.3 \\
4.0 \\
3.6\end{array}$ \\
\hline $\begin{array}{l}- \\
- \\
-\end{array}$ & $\begin{array}{l}3.7 \\
3.8 \\
5.4 \\
1.2 \\
5.2\end{array}$ \\
\hline - & 5.3 \\
\hline- & 4.3 \\
\hline- & 4.7 \\
\hline
\end{tabular}

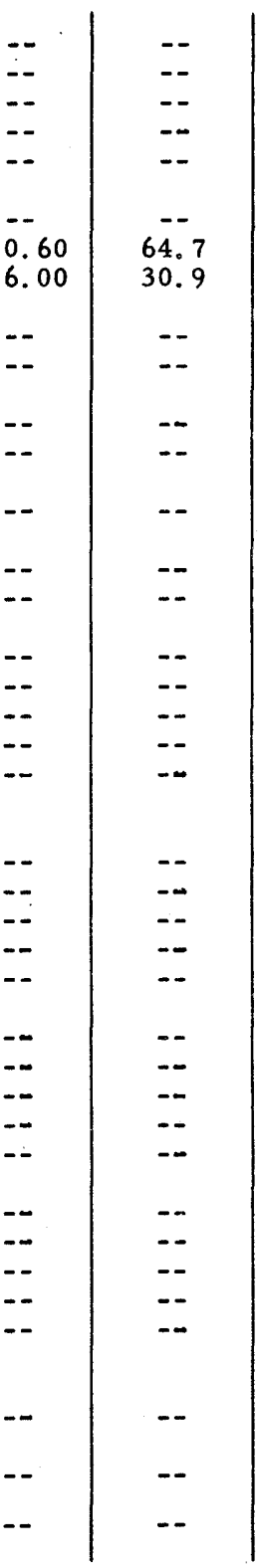

95.5 99.4
101.6 110.3

123.2

128.5

132.2

135.6

141.2

144.2

146.5

148.5

150.4

152.3

154.0

155.9

157.4

161.3
162.8

164.7

165.7

166.2

167.2

168.2

170.0

171.8

174.1

178.1

181.7

185.4

189.2

194.6

195.8

201.0

206. 3

210.6

215.3

-
-
-
-
-
-
-
-
-
-
-
-
-
-
-
-
-
-
-

-
-
-
-
-
-
-
-
-
-
-
-
-
-

1 Fossil collection made by J. E. Smedley, Paleontology and Stratigraphy Branch, U. S. Geological Survey. 
Franson Canyon-Continued

\begin{tabular}{|c|c|c|c|c|c|c|c|}
\hline \multirow{2}{*}{$\begin{array}{l}\text { Bed } \\
\text { no. }\end{array}$} & \multirow{2}{*}{ Rock description } & \multirow{2}{*}{$\begin{array}{l}\text { Sample } \\
\text { no. }\end{array}$} & \multirow{2}{*}{$\begin{array}{c}\text { Thickness } \\
\text { (feet) }\end{array}$} & \multicolumn{2}{|c|}{$\begin{array}{c}\text { Chemical analyses } \\
\text { (percent) }\end{array}$} & \multirow{2}{*}{$\begin{array}{c}\text { Cumulative } \\
\text { thickness } \\
\text { (feet) }\end{array}$} & \multirow{2}{*}{$\begin{array}{l}\text { Thickness } \mathrm{x} \\
\text { percent } \mathrm{P}_{2} \mathrm{O}_{5} \\
\text { (cumulative) }\end{array}$} \\
\hline & & & & $\mathrm{P}_{2} \mathrm{O}_{5}$ & $\begin{array}{l}\text { Acid } \\
\text { insoluble }\end{array}$ & & \\
\hline $\begin{array}{l}U-7 \\
U-6 \\
U-5 \\
U-4 \\
U-3\end{array}$ & $\begin{array}{l}\text { Siltstone, carbonatic; fos. col. no. } 12494 \\
\text { Siltstone, carbonatic; fos. col. no. } 12494 \\
\text { Siltstone; fos. col. no. } 12494 \\
\text { Covered interval; fos. col. no. } 12494 \\
\text { Carbonate rock; fos. col. no. } 12494\end{array}$ & $\begin{array}{l}-- \\
-- \\
-- \\
--\end{array}$ & $\begin{array}{l}1.4 \\
1.1 \\
1.1 \\
7.6 \\
2.5\end{array}$ & $\begin{array}{l}-- \\
-- \\
-- \\
-- \\
--\end{array}$ & $\begin{array}{l}-- \\
-- \\
-- \\
--\end{array}$ & $\begin{array}{l}216.7 \\
217.8 \\
218.9 \\
226.5 \\
229.0\end{array}$ & $\begin{array}{l}-- \\
-- \\
-- \\
--\end{array}$ \\
\hline $\begin{array}{l}U-2 \\
U-1\end{array}$ & $\begin{array}{l}\text { Carbonate rock, argillaceous; fos. col. } \\
\text { no. } 12493- \\
\text { Limestone, argillaceous }\end{array}$ & -- & $\begin{array}{r}12.9 \\
1.7\end{array}$ & -- & -- & $\begin{array}{l}241.9 \\
243.6\end{array}$ & -- \\
\hline
\end{tabular}

Phosphatic shale member of Park City formation (?)

\begin{tabular}{|c|c|}
\hline $\begin{array}{l}P-50 \\
P-49 \\
P-48 \\
P-47\end{array}$ & $\begin{array}{l}\text { Siltstone, carbonatic } \\
\text { Mudstone, carbonatic; fos. col. no. } 12492 \\
\text { Carbonate rock, cherty } \\
\text { Carbonate rock, phosphatic; fos. col. no. } \\
12492\end{array}$ \\
\hline$P-46$ & Carbonate rock, argillaceous \\
\hline $\begin{array}{l}P-45 \\
P-44 \\
P-43 \\
P-42 \\
P-41\end{array}$ & $\begin{array}{l}\text { Carbonate rock; fos. col. no. } 12491 \\
\text { Siltstone, cherty, carbonatic } \\
\text { Mudstone, carbonatic } \\
\text { Siltstone, cherty, carbonatic } \\
\text { Carbonate rock }\end{array}$ \\
\hline $\begin{array}{l}P-40 \\
P-39 \\
P-38 \\
P-37 \\
P-36\end{array}$ & $\begin{array}{l}\text { te rock, cherty } \\
\text { ne, phosphatic, carbonatic } \\
\text { ate rock, argillaceous } \\
\text { ne, carbonatic, phosphatic } \\
\text { ne, carbonatic }\end{array}$ \\
\hline $\begin{array}{l}P-35 \\
P-34 \\
P-33 \\
P-32 \\
P-31\end{array}$ & $\begin{array}{l}\text { Carbonate rock } \\
\text { Carbonate rock } \\
\text { Carbonate rock } \\
\text { Carbonate rock } \\
\text { Carbonate rock }\end{array}$ \\
\hline $\begin{array}{l}P-30 \\
P-29 \\
P-28 \\
P-27 \\
P-26\end{array}$ & $\begin{array}{l}\text { Muc } \\
\text { Car } \\
\text { Silt } \\
\text { Silt }\end{array}$ \\
\hline
\end{tabular}

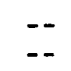

6317 - RAS 6316- RAS

6315- RAS 6314- RAS

6313- RAS

$--$

$-$

$-$

$6312-$ RAS

6311- RAS

6310- RAS

6309- RAS

--
--
--
--
--
--
--
--
--
--

2.3
.5

1.5

1.0

1. 1

2.3

1.3

1. 5

5. 4

1. 1

1. 5

1.8

3. 4

2. 9

2. 3

1. 1

1.7

1.8

1. 3

1. 7

4.2
1.8

\begin{tabular}{l|c|}
-- & -- \\
-- & -- \\
7.85 & -- \\
2.80 & 8.7 \\
7.20 & 12.1 \\
2.15 & 47.8 \\
4.30 & 45.9 \\
-- & -- \\
-- & -- \\
& \\
12.6 & -- \\
2.95 & 39.2 \\
9.80 & 47.5 \\
2.65 & 68.8 \\
-- & -- \\
-- & -- \\
-- & -- \\
-- & -- \\
-- & -- \\
-- & -- \\
-- & -- \\
-- & -- \\
-- & -- \\
& -- \\
\hline- &
\end{tabular}

2. 3

2. 8

$--$

$-\infty$

4. 9

7.85

6.0

10.93

6. 3

8.9
9.3

9.3

10.6

12.1

17.5

18.6

20.1

20.9

21.9

25.3

28.2

30.5

31.6
33.3

35.1

36.4

36.4
38.1

38.1
42.3

44.1

13.09

18.68

20.40

--

--

13. 86

18.28

26.12

28,78

$-$

$-$

$-$

--

$-$

$-$

$-$

$-$ 


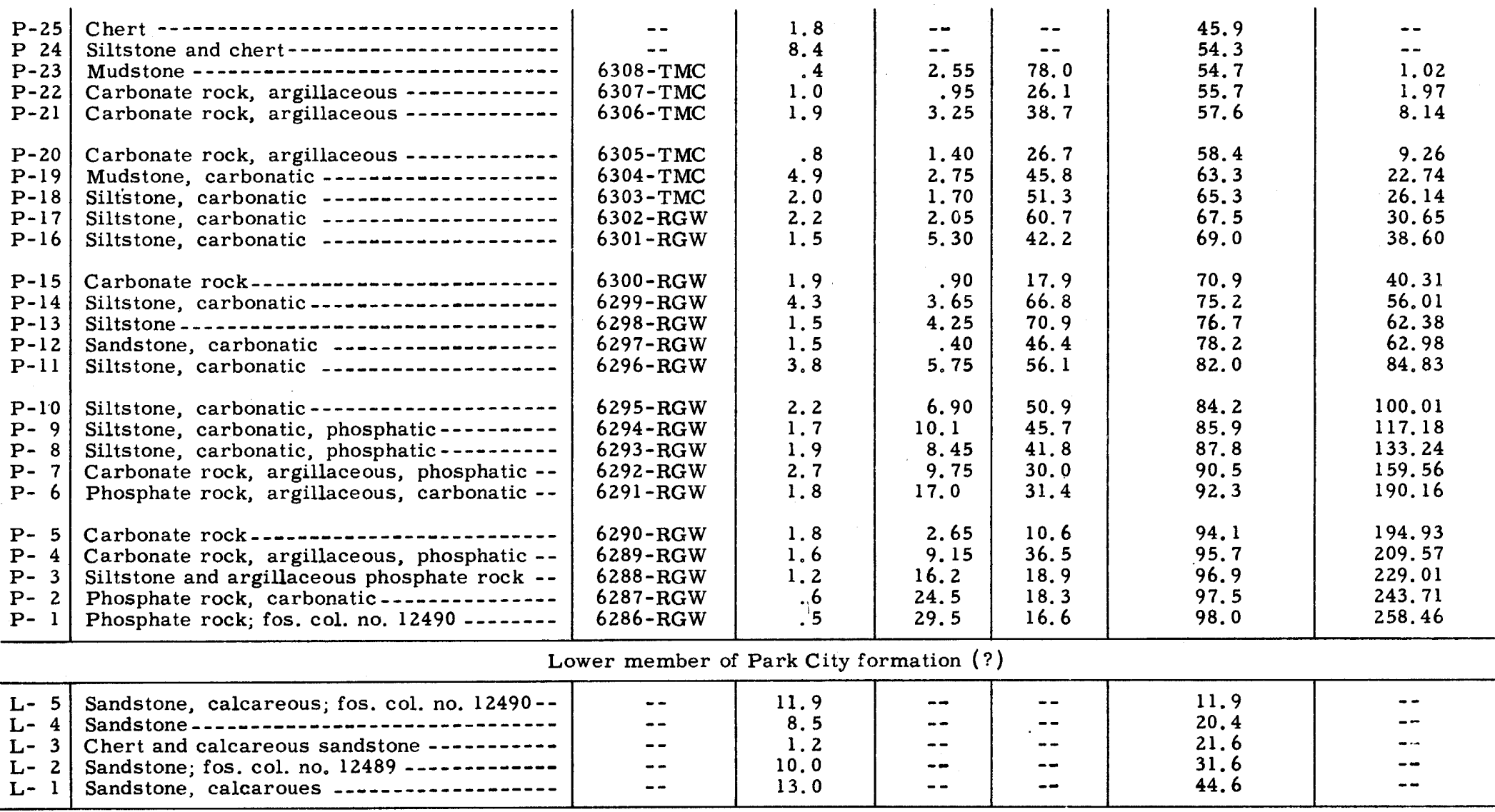


Park City formation measured and sampled in hand trenches and at natural exposure in Horseshoe Canyon on the north side of the Green River, sec. 36, T. 3 N., R. 20 E., Daggatt County, Utah. Beds strike S. $50^{\circ}$ E. and dip $15^{\circ} \mathrm{N}$. Section measured and sampled by R. G. Waring, R. A. Smart, H. W. Peirce, and T. M. Cheney in September 1950. Samples analyzed by U. S. Bureau of Mines laboratory, Albany, Oreg.

\begin{tabular}{|c|c|c|c|c|c|c|c|}
\hline \multirow{2}{*}{$\begin{array}{c}\text { Bed } \\
\text { no. }\end{array}$} & \multirow{2}{*}{ Rock description } & \multirow{2}{*}{$\begin{array}{l}\text { Sample } \\
\text { no. }\end{array}$} & \multirow{2}{*}{$\begin{array}{c}\text { Thickness } \\
\text { (feet) }\end{array}$} & \multicolumn{2}{|c|}{$\begin{array}{c}\text { Chemical analyses } \\
\text { (percent) }\end{array}$} & \multirow{2}{*}{$\begin{array}{l}\text { Cumulative } \\
\text { thickness } \\
\text { (feet) }\end{array}$} & \multirow{2}{*}{$\begin{array}{l}\text { Thickness } \mathrm{x} \\
\text { percent } \mathrm{P}_{2} \mathrm{O}_{5} \\
\text { (cumulative) }\end{array}$} \\
\hline & & & & $\mathrm{P}_{2} \mathrm{O}_{5}$ & $\begin{array}{c}\text { Acid } \\
\text { insoluble }\end{array}$ & & \\
\hline \multicolumn{8}{|c|}{ Upper member of Park City formation_top not exposed } \\
\hline $\begin{array}{l}U-30 \\
U-29 \\
U-28 \\
U-27 \\
U-26\end{array}$ & $\begin{array}{l}\text { Carbonate rock; fos. col. no. } 12287^{1} \\
\text { Carbonate rock; fos. col, no. } 12287 \\
\text { Carbonate rock, argillaceous } \\
\text { Carbonate rock } \\
\text { Carbonate rock; fos. col. no. } 12286\end{array}$ & $\begin{array}{l}-- \\
-- \\
-- \\
--\end{array}$ & $\begin{array}{l}4.0 \\
3.6 \\
2.6 \\
15.0 \\
25\end{array}$ & $\begin{array}{l}-- \\
-- \\
-- \\
--\end{array}$ & $\begin{array}{l}-- \\
-- \\
-- \\
--\end{array}$ & $\begin{array}{r}4.0 \\
7.6 \\
10.2 \\
25.2 \\
50.2\end{array}$ & $\begin{array}{l}-- \\
-- \\
-- \\
--\end{array}$ \\
\hline $\begin{array}{l}U-25 \\
U-24 \\
U-23 \\
U-22 \\
U-21\end{array}$ & $\begin{array}{l}\text { Carbonate rock; fos. col. no. } 12286 \\
\text { Carbonate rock; fos. col. no. } 12286 \\
\text { Carbonate rock } \\
\text { Carbonate rock, argillaceous } \\
\text { Carbonate rock }\end{array}$ & $\begin{array}{l}-- \\
-- \\
-- \\
--\end{array}$ & $\begin{array}{l}4.1 \\
7.3 \\
6.3 \\
9.0 \\
4.2\end{array}$ & $\begin{array}{l}-- \\
-- \\
-- \\
-- \\
--\end{array}$ & $\begin{array}{l}-- \\
-- \\
-- \\
--\end{array}$ & $\begin{array}{l}54.3 \\
61.6 \\
67.9 \\
76.9 \\
81.1\end{array}$ & $\begin{array}{l}-- \\
-- \\
-- \\
--\end{array}$ \\
\hline $\begin{array}{l}U-20 \\
U-19\end{array}$ & $\begin{array}{l}\text { Carbonate rock } \\
\text { Covered interval; dominantly carbonate }\end{array}$ & -- & 4.2 & -- & - & 85.3 & -- \\
\hline $\begin{array}{l}U-18 \\
U-17 \\
U-16\end{array}$ & $\begin{array}{l}\text { Gypsum } \\
\text { Mudstone, carbonatic, gypsiferous } \\
\text { Sandstone, carbonatic }\end{array}$ & $5305-\overline{R G W}$ & $\begin{array}{l}1.0 \\
1.8 \\
3.6\end{array}$ & -- & 68 & $\begin{array}{l}111.3 \\
112.3 \\
114.1 \\
117.7\end{array}$ & $\begin{array}{l}-- \\
-- \\
0.72\end{array}$ \\
\hline $\begin{array}{l}U-15 \\
U-14 \\
U-13 \\
U-12 \\
U-11\end{array}$ & $\begin{array}{l}\text { Sandstone, carbonatic } \\
\text { Sandstone } \\
\text { Sandstone } \\
\text { Sandstone } \\
\text { Sandstone, carbonatic }\end{array}$ & $\begin{array}{l}5304-\text { RGW } \\
5303-\text { RGW } \\
5302-\text { RGW } \\
5301-\text { RGW } \\
--\end{array}$ & $\begin{array}{l}1.9 \\
2.9 \\
2.1 \\
3.1 \\
4.0\end{array}$ & $\begin{array}{r}.2 \\
.2 \\
.2 \\
.4 \\
--\end{array}$ & $\begin{array}{l}68.0 \\
74.5 \\
73.6 \\
70.7 \\
--\end{array}$ & $\begin{array}{l}119.6 \\
122.5 \\
124.6 \\
127.7 \\
131.7\end{array}$ & $\begin{array}{l}1.10 \\
1.68 \\
2.10 \\
3.34 \\
--\end{array}$ \\
\hline $\begin{array}{l}U-10 \\
U-9 \\
U-8 \\
U-7 \\
U-6\end{array}$ & $\begin{array}{l}\text { Carbonate rock, sandy; fos. col. no. } 12285-- \\
\text { Carbonate rock, sandy; fos. col. no. } 12285- \\
\text { Sandstone, carbonatic; fos. col. no. } 12285- \\
\text { Carbonate rock; fos. col. no. } 12284 \\
\text { Mudstone, cherty }\end{array}$ & $\begin{array}{l}\text { 5300-RGW } \\
5299-\text { RGW } \\
-- \\
-- \\
--\end{array}$ & $\begin{array}{r}3.3 \\
2.9 \\
12.0 \\
3.2 \\
4.7\end{array}$ & $\begin{array}{l}1.1 \\
1.0 \\
-- \\
-- \\
--\end{array}$ & $\begin{array}{l}41.3 \\
42.9 \\
-- \\
-- \\
--\end{array}$ & $\begin{array}{l}135.0 \\
137.9 \\
149.9 \\
153.1 \\
157.8\end{array}$ & $\begin{array}{l}-- \\
-- \\
-- \\
--\end{array}$ \\
\hline $\begin{array}{l}U-5 \\
U-4 \\
U-3 \\
U-2 \\
U-1\end{array}$ & $\begin{array}{l}\text { Carbonate rock } \\
\text { Gypsum } \\
\text { Mudstone } \\
\text { Carbonate rock } \\
\text { Carbonate rook; fos. col. no. } 12283\end{array}$ & $\begin{array}{l}-- \\
-- \\
-- \\
--\end{array}$ & $\begin{array}{r}5.2 \\
.3 \\
.5 \\
4.0 \\
7.6\end{array}$ & $\begin{array}{l}-- \\
-- \\
-- \\
--\end{array}$ & $\begin{array}{l}-- \\
-- \\
-- \\
-- \\
--\end{array}$ & $\begin{array}{l}163.0 \\
163.3 \\
163.8 \\
167.8 \\
175.4\end{array}$ & $\begin{array}{l}-- \\
-- \\
-- \\
--\end{array}$ \\
\hline
\end{tabular}


Phosphatic shale member of Park City formation

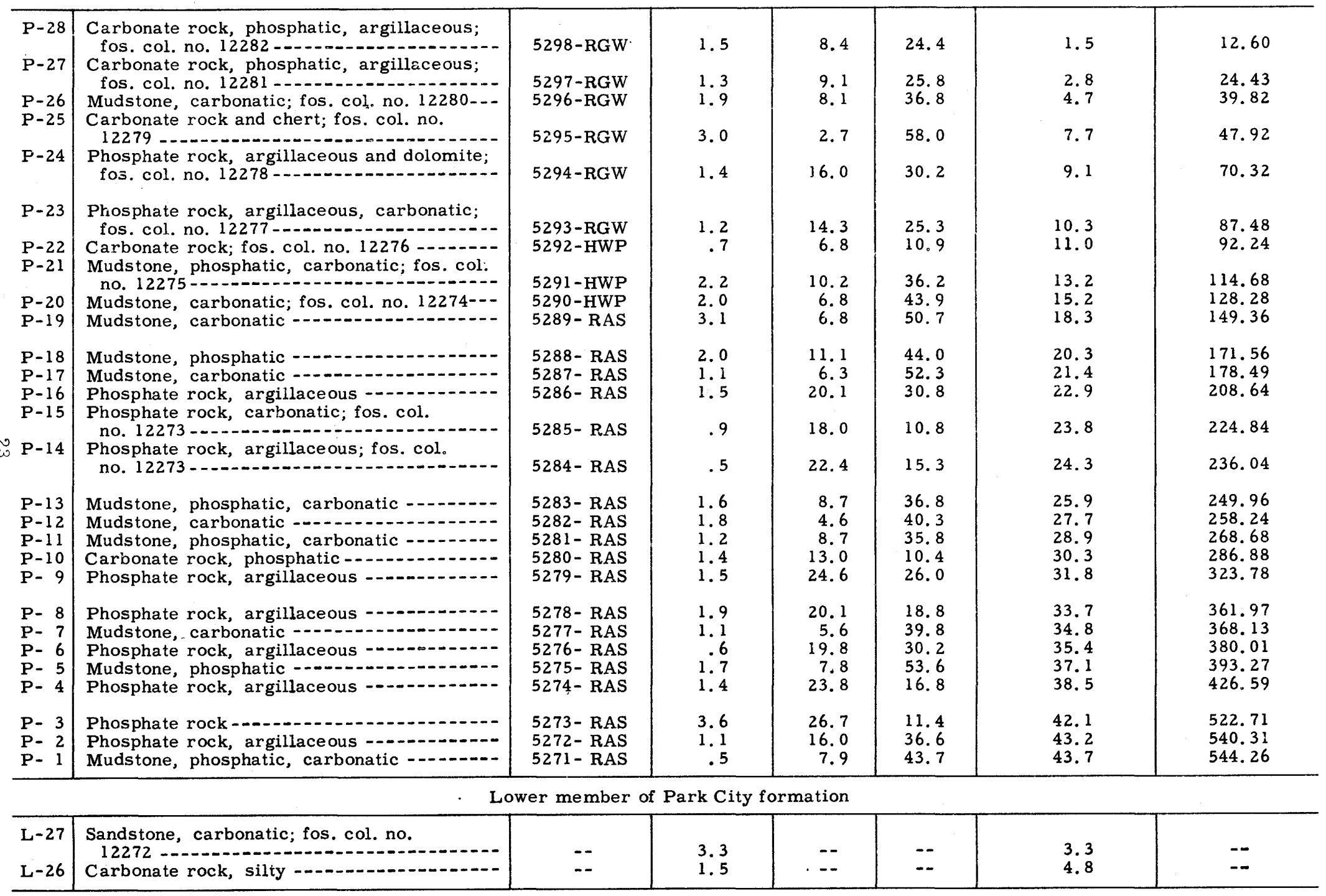

${ }^{l}$ Fossil collection made by J. E. Smedley, Paleontology and Stratigraphy Branch, U. S. Geological Survey. 
Horseshoe Canyon-Continued

\begin{tabular}{|c|c|c|c|c|c|c|c|}
\hline \multirow{2}{*}{$\begin{array}{c}\text { Bed } \\
\text { no. }\end{array}$} & \multirow{2}{*}{ Rock description } & \multirow{2}{*}{$\begin{array}{l}\text { Sample } \\
\text { no. }\end{array}$} & \multirow{2}{*}{$\begin{array}{c}\text { Thickness } \\
\text { (feet) }\end{array}$} & \multicolumn{2}{|c|}{$\begin{array}{c}\text { Chemical analyses } \\
\text { (percent) }\end{array}$} & \multirow{2}{*}{$\begin{array}{c}\text { Cumulative } \\
\text { thickness } \\
\text { (feet) }\end{array}$} & \multirow{2}{*}{$\begin{array}{l}\text { Thickness } x \\
\text { percent } \mathrm{P}_{2} \mathrm{O}_{5} \\
\text { (cumulative) }\end{array}$} \\
\hline & & & & $\mathrm{P}_{2} \mathrm{O}_{5}$ & $\begin{array}{c}\text { Acid } \\
\text { insoluble }\end{array}$ & & \\
\hline $\begin{array}{l}L-25 \\
L-24 \\
L-23 \\
L-22 \\
L-21\end{array}$ & $\begin{array}{l}\text { Sandstone, carbonatic } \\
\text { Carbonate rock, quartzitic; fos. col. no. } \\
12271 \\
\text { Sandstone, carbonatic } \\
\text { Covered interval } \\
\text { Sandstone, carbonatic }\end{array}$ & $\begin{array}{l}-- \\
-- \\
-- \\
--\end{array}$ & $\begin{array}{r}1.4 \\
4.3 \\
20.6 \\
4.6 \\
10.8\end{array}$ & $\begin{array}{l}-- \\
-- \\
-- \\
--\end{array}$ & $\begin{array}{l}-- \\
-- \\
-- \\
--\end{array}$ & $\begin{array}{l}6.2 \\
10.5 \\
31.1 \\
35.1 \\
45.9\end{array}$ & $\begin{array}{l}-- \\
-- \\
-- \\
--\end{array}$ \\
\hline $\begin{array}{l}L-20 \\
L-19 \\
L-18 \\
L-17 \\
L-16\end{array}$ & $\begin{array}{l}\text { Sandstone, carbonatic } \\
\text { Carbonate rock and carbonatic mudstone } \\
\text { Carbonate rock } \\
\text { Sandstone, carbonatic } \\
\text { Sandstone, carbonatic }\end{array}$ & $\begin{array}{l}-- \\
-- \\
-- \\
--\end{array}$ & $\begin{array}{r}5.3 \\
.8 \\
1.3 \\
1.2 \\
16.8\end{array}$ & $\begin{array}{l}-- \\
-- \\
-- \\
--\end{array}$ & $\begin{array}{l}-- \\
-- \\
-- \\
--\end{array}$ & $\begin{array}{l}51.2 \\
52.0 \\
53.3 \\
54.5 \\
71.3\end{array}$ & $\begin{array}{l}-\infty \\
-\infty \\
-- \\
--\end{array}$ \\
\hline $\begin{array}{l}\mathrm{L}-15 \\
\mathrm{~L}-14 \\
\mathrm{~L}-13 \\
\mathrm{~L}-12 \\
\mathrm{~L}-11\end{array}$ & $\begin{array}{l}\text { Carbonate rock, sandy } \\
\text { Carbonate rock } \\
\text { Siltstone, carbonatic } \\
\text { Carbonate rock } \\
\text { Carbonate rock; fos. col. no. } 12270\end{array}$ & $\begin{array}{l}-- \\
-- \\
-- \\
--\end{array}$ & $\begin{array}{r}6.3 \\
.4 \\
1.1 \\
.3 \\
2.2\end{array}$ & $\begin{array}{l}-- \\
-- \\
-- \\
--\end{array}$ & $\begin{array}{l}-- \\
-- \\
-- \\
--\end{array}$ & $\begin{array}{l}77.6 \\
78.0 \\
79.1 \\
79.4 \\
81.6\end{array}$ & $\begin{array}{l}-- \\
-- \\
-- \\
--\end{array}$ \\
\hline $\begin{array}{l}L-10 \\
L=9 \\
L-8 \\
L-7 \\
L-6\end{array}$ & $\begin{array}{l}\text { Sandstone, carbonatic } \\
\text { Carbonate rock, sandy } \\
\text { Carbonate rock, sandy } \\
\text { Carbonate rock, quartzitic } \\
\text { Sandstone and carbonate rock }\end{array}$ & $\begin{array}{l}-- \\
-- \\
-- \\
--\end{array}$ & $\begin{array}{r}3.5 \\
3.2 \\
8.2 \\
2.8 \\
.9\end{array}$ & $\begin{array}{l}-- \\
-- \\
-- \\
--\end{array}$ & $\begin{array}{l}-- \\
-- \\
-- \\
--\end{array}$ & $\begin{array}{r}85.1 \\
88.3 \\
96.5 \\
99.3 \\
100.2\end{array}$ & $\begin{array}{l}-- \\
-- \\
-- \\
--\end{array}$ \\
\hline $\begin{array}{l}L-5 \\
L-4 \\
L-3 \\
L-2 \\
L-1\end{array}$ & $\begin{array}{l}\text { Carbonate rock, sandy } \\
\text { Sandstone, carbonatic } \\
\text { Carbonate rock, sandy } \\
\text { Sandstone, carbonatic } \\
\text { Carbonate rock, sandy }\end{array}$ & $\begin{array}{l}-- \\
-- \\
-- \\
--\end{array}$ & $\begin{array}{l}1.0 \\
.5 \\
4.3 \\
1.4 \\
1.2\end{array}$ & $\begin{array}{l}-- \\
-- \\
-- \\
-- \\
--\end{array}$ & $\begin{array}{l}-- \\
-- \\
-- \\
-- \\
--\end{array}$ & $\begin{array}{l}101.2 \\
101.7 \\
106.0 \\
107.4 \\
108.6\end{array}$ & $\begin{array}{l}-- \\
-- \\
-- \\
-- \\
--\end{array}$ \\
\hline \multicolumn{8}{|c|}{ Weber formation - top bed only } \\
\hline Cw-1 & Sandstone, carbonatic & -- & 6.0 & $-\infty$ & -- & 6.0 & -- \\
\hline
\end{tabular}


Part of Park City formation measured and sampled in outcrop and bulldozer trench near Fort Douglas, E $\frac{1}{2}$ NE $\frac{1}{4}$ sec. 17 , T. 1 N., R. 2 E., Salt Lake County, Utah. Beds strike N. $73^{\circ}$ E. and dip $68^{\circ} \mathrm{S}$. Section measured and sampled by M. A. Warner, R. A. Smart, and T. M. Cheney. Samples analyzed by Trace Elements Section laboratory, Denver, Colo.

\begin{tabular}{c|c|c|c|c|c|c}
\hline $\begin{array}{c}\text { Bed } \\
\text { no. }\end{array}$ & Rock description & Sample & \multirow{2}{*}{$\begin{array}{c}\text { Thickness } \\
\text { (feet) }\end{array}$} & \multicolumn{2}{c|}{$\begin{array}{c}\text { Chemical analyses } \\
\text { (percent) }\end{array}$} & $\begin{array}{c}\text { Cumulative } \\
\text { thickness } \\
\text { (feet) }\end{array}$ \\
\end{tabular}

Phosphatic shale member of Park City formation (?)

\begin{tabular}{|c|c|}
\hline $\begin{array}{l}P-242 \\
P-241 \\
P-240 \\
P-239 \\
P-238\end{array}$ & $\begin{array}{l}\text { Silts tone } \\
\text { Siltstone } \\
\text { Mudstone } \\
\text { Mudstone } \\
\text { Chert }\end{array}$ \\
\hline $\begin{array}{l}P-237 \\
P-236 \\
P-235 \\
P-234 \\
P-233\end{array}$ & $\begin{array}{l}\text { Mudstone, cherty } \\
\text { Mudstone, cherty } \\
\text { Chert, argillaceous } \\
\text { Siltstone } \\
\text { Chert, argillaceous }\end{array}$ \\
\hline $\begin{array}{l}P-232 \\
P-231 \\
P-230 \\
P-229 \\
P-228\end{array}$ & $\begin{array}{l}\text { Chert, argillaceous } \\
\text { Shear zone; brecciated siltstone } \\
\text { Siltstone } \\
\text { Mudstone } \\
\text { Carbonate rock }\end{array}$ \\
\hline $\begin{array}{l}P-227 \\
P-226 \\
P-225 \\
P-224 \\
P-223\end{array}$ & $\begin{array}{l}\text { Phosphate rock, argillaceous } \\
\text { Siltstone, carbonatic } \\
\text { Mudstone, cherty } \\
\text { Mudstone } \\
\text { Siltstone }\end{array}$ \\
\hline $\begin{array}{l}P-222 \\
P-221 \\
P-220 \\
P-219 \\
P-218\end{array}$ & $\begin{array}{l}\text { Siltstone } \\
\text { Mudstone } \\
\text { Siltstone } \\
\text { Chert } \\
\text { Mudstone }\end{array}$ \\
\hline $\begin{array}{l}P-217 \\
P-216 \\
P-215 \\
P-214 \\
P-213\end{array}$ & $\begin{array}{l}\text { Mudstone, cherty } \\
\text { Mudstone } \\
\text { Mudstone } \\
\text { Mudstone } \\
\text { Mudstone, phosphatic }\end{array}$ \\
\hline $\begin{array}{l}P-212 \\
P-211\end{array}$ & $\begin{array}{l}\text { Mudstone } \\
\text { Mudstone and phosphate rock }\end{array}$ \\
\hline
\end{tabular}

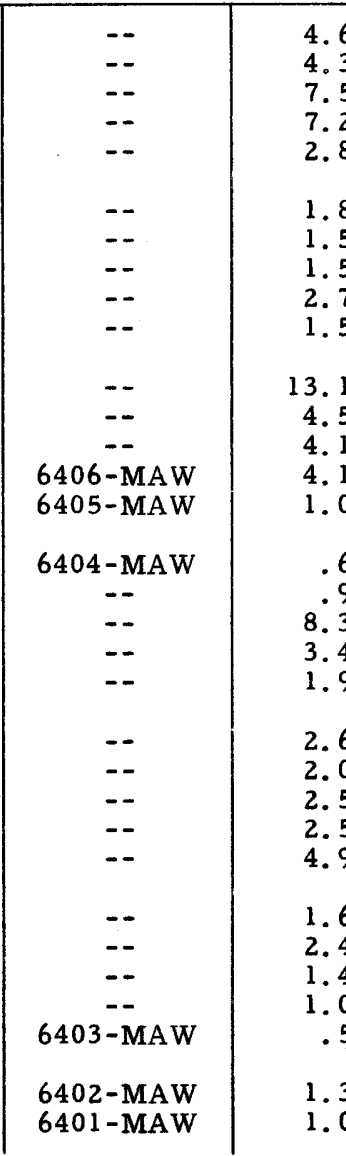

\begin{tabular}{l|}
4.6 \\
4.3 \\
7.5 \\
7.2 \\
2.8 \\
1.8 \\
1.5 \\
1.5 \\
2.7 \\
1.5 \\
3.1 \\
4.5 \\
4.1 \\
4.1 \\
1.0 \\
.6 \\
.9 \\
8.3 \\
3.4 \\
1.9 \\
2.6 \\
2.0 \\
2.5 \\
2.5 \\
4.9 \\
1.6 \\
2.4 \\
1.4 \\
1.0 \\
.5 \\
1.3 \\
1.0 \\
\end{tabular}

\begin{tabular}{c|c|}
-- & -- \\
-- & -- \\
-- & -- \\
-- & -- \\
-- & -- \\
-- & -- \\
-- & -- \\
-- & -- \\
- & -- \\
-- & -- \\
-- & -- \\
-- & -- \\
6.50 & 64.2 \\
1.70 & 6.90 \\
20.9 & 30.0 \\
-- & -- \\
-- & -- \\
-- & -- \\
-- & -- \\
-- & -- \\
-- & -- \\
-- & -- \\
-- & -- \\
-- & -- \\
-- & -- \\
-- & -- \\
-- & -- \\
17.3 & 44.9 \\
1.20 & 86.9 \\
15.5 & 51.7 \\
\hline- \\
\hline-
\end{tabular}

4.6
8.9
16.4
23.6
26.4
28.2
29.7
31.2
33.9
35.2
48.5
53.0
57.1
61.2
62.2
62.8
63.7
72.0
75.4
77.3
79.9
81.9
84.4
86.9
91.8
93.4
95.8
97.2
98.2
98.7
100.0
101.0

$--$

$\overline{--}$

$--$

$--$

$--$

$--$

(3).

35.2

8.5

53.0

1.2

62.2

62.8

63. 7

75.4

77.3

79.9

1.9

8.

91.8

93.4

98.2

98.7

--

$-$

$-$

$--$

$--$

--

--

$--$ 
Fort Douglas - Continued

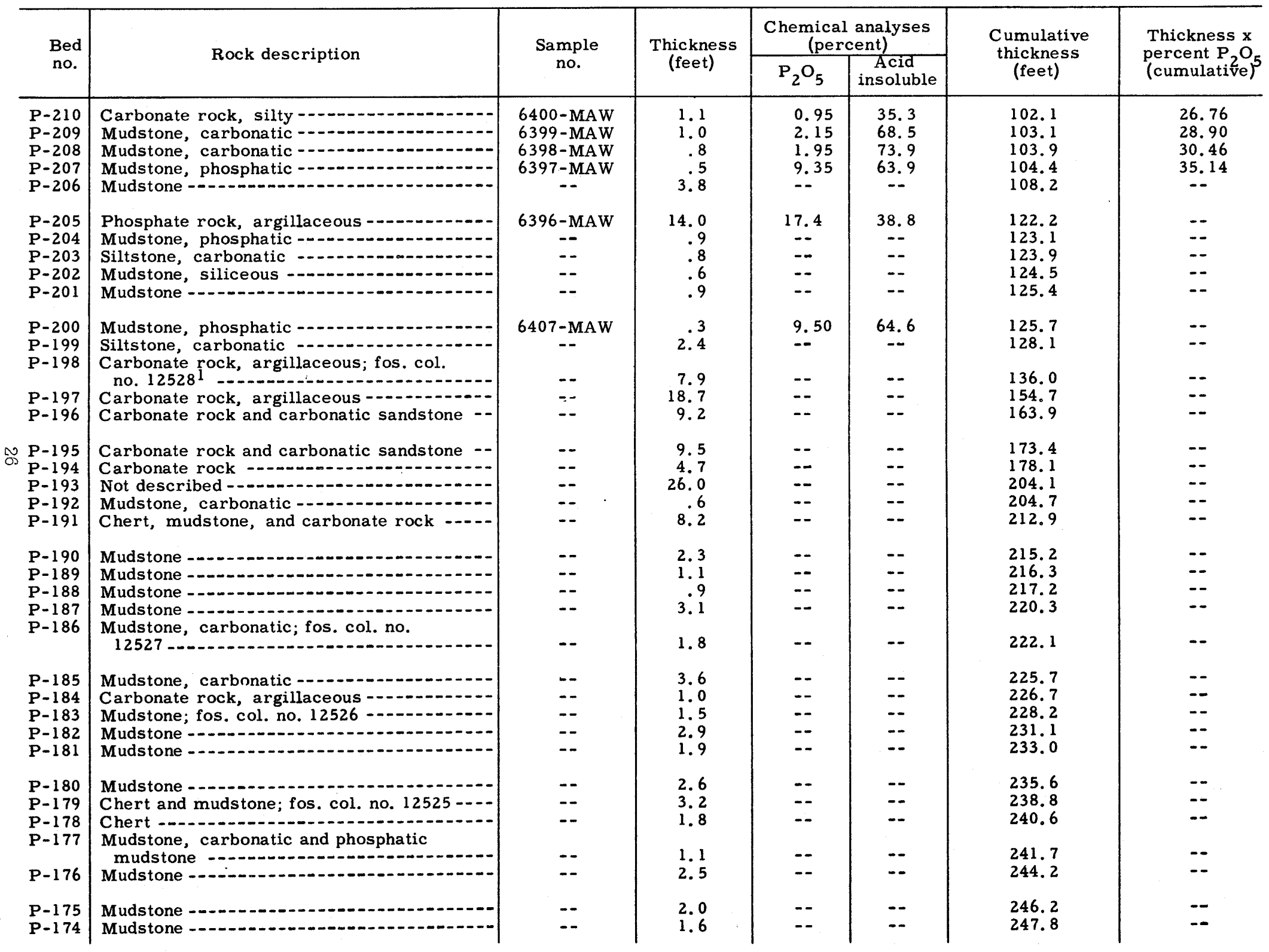




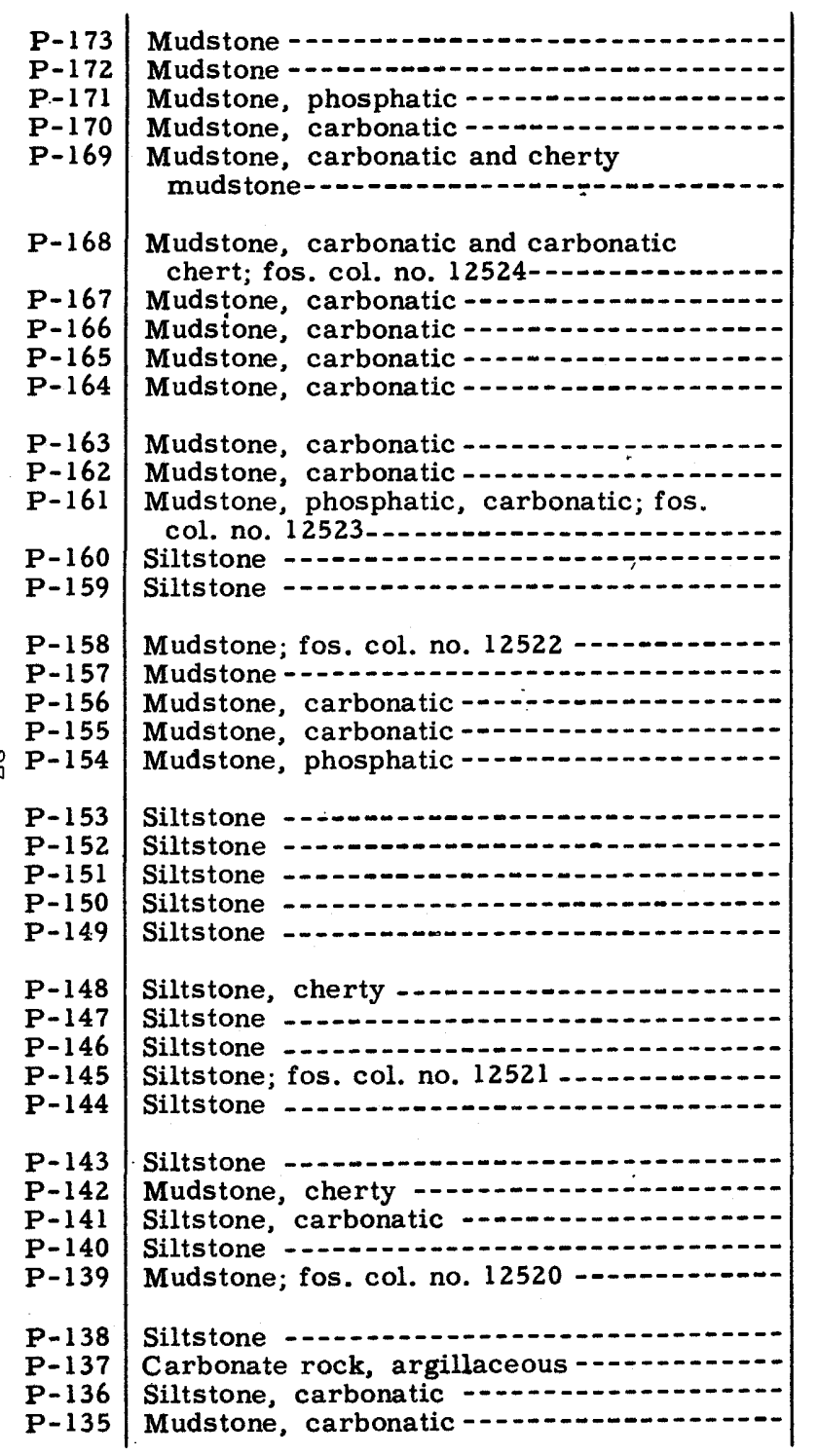

\begin{tabular}{|c|c|c|c|c|}
\hline $\begin{array}{l}\text { 6395-- TMC } \\
\text { 6394- TMC } \\
\text { 6393- TMC }\end{array}$ & $\begin{array}{r}4.2 \\
1.9 \\
1.9 \\
.8\end{array}$ & $\begin{array}{l}-- \\
1.35 \\
9.90 \\
1.85\end{array}$ & $\begin{array}{l}-- \\
82.7 \\
55.8 \\
66.3\end{array}$ & $\begin{array}{l}252.0 \\
253.9 \\
255.8 \\
256.6\end{array}$ \\
\hline 6392-TMC & .8 & 5.85 & 58.0 & 257.4 \\
\hline $\begin{array}{l}\text { 6391- TMC } \\
6390-\text { TMC } \\
\text { 6389- TMC } \\
\text { 6388- TMC } \\
6387-\text { TMC }\end{array}$ & $\begin{array}{l}2.6 \\
2.9 \\
1.5 \\
2.9 \\
1.6\end{array}$ & $\begin{array}{l}1.90 \\
1.70 \\
1.70 \\
2.25 \\
2.50\end{array}$ & $\begin{array}{l}63.6 \\
54.1 \\
52.1 \\
52.7 \\
61.0\end{array}$ & $\begin{array}{l}260.0 \\
262.9 \\
264.4 \\
267.3 \\
268.9\end{array}$ \\
\hline $\begin{array}{l}\text { 6386- TMC } \\
6385-\text { TMC }\end{array}$ & $\begin{array}{l}1.1 \\
1.9\end{array}$ & $\begin{array}{l}2.75 \\
2.75\end{array}$ & $\begin{array}{l}55.5 \\
48.2\end{array}$ & $\begin{array}{l}270.0 \\
271.9\end{array}$ \\
\hline $\begin{array}{c}\text { 6384- TMC } \\
--\end{array}$ & $\begin{array}{l}.6 \\
5.2 \\
7.3\end{array}$ & $\begin{array}{c}13.3 \\
--\end{array}$ & $\begin{array}{c}46.6 \\
-- \\
--\end{array}$ & $\begin{array}{l}272.5 \\
277.7 \\
285.0\end{array}$ \\
\hline $\begin{array}{c}-- \\
-- \\
6383-\text { MAW } \\
6382-\text { MAW } \\
6381-\text { MAW }\end{array}$ & $\begin{array}{r}3.8 \\
4.5 \\
.4 \\
1.4 \\
.3\end{array}$ & $\begin{array}{l}-- \\
-- \\
4.65 \\
2.50 \\
15.5\end{array}$ & $\begin{array}{c}-- \\
-- \\
52.0 \\
47.5 \\
47.9\end{array}$ & $\begin{array}{l}288.8 \\
293.3 \\
293.7 \\
295.1 \\
295.4\end{array}$ \\
\hline $\begin{array}{l}-- \\
-- \\
-- \\
--\end{array}$ & $\begin{array}{r}10.0 \\
3.3 \\
5.8 \\
8.0 \\
2.0\end{array}$ & $\begin{array}{l}-- \\
-- \\
-- \\
--\end{array}$ & $\begin{array}{l}-- \\
-- \\
-- \\
--\end{array}$ & $\begin{array}{l}305.4 \\
308.7 \\
314.5 \\
322.5 \\
324.5\end{array}$ \\
\hline $\begin{array}{l}-- \\
-- \\
-- \\
--\end{array}$ & $\begin{array}{l}3.8 \\
2.2 \\
3.2 \\
7.3 \\
1.8\end{array}$ & $\begin{array}{l}-- \\
-- \\
-- \\
--\end{array}$ & $\begin{array}{l}-- \\
-- \\
-- \\
-- \\
--\end{array}$ & $\begin{array}{l}328.3 \\
330.5 \\
333.7 \\
341.0 \\
342.8\end{array}$ \\
\hline $\begin{array}{c}-- \\
-- \\
-- \\
-- \\
6380-\mathrm{MAW}\end{array}$ & $\begin{array}{r}1.5 \\
.5 \\
1.0 \\
.7 \\
.8\end{array}$ & $\begin{array}{l}-- \\
-- \\
-- \\
-- \\
4.70\end{array}$ & $\begin{array}{l}-- \\
-- \\
-- \\
-- \\
79.5\end{array}$ & $\begin{array}{l}344.3 \\
344.8 \\
345.8 \\
346.5 \\
347.3\end{array}$ \\
\hline $\begin{array}{l}\text { 6379-MAW } \\
6378-\text { MAW } \\
6377-\text { MAW } \\
6376-\text { MAW }\end{array}$ & $\begin{array}{l}3.4 \\
5.2 \\
1.2 \\
1.4\end{array}$ & $\begin{array}{r}2.35 \\
1.00 \\
.85 \\
2.80\end{array}$ & $\begin{array}{l}78.0 \\
45.7 \\
64.7 \\
65.8\end{array}$ & $\begin{array}{l}350.7 \\
355.9 \\
357.1 \\
358.5\end{array}$ \\
\hline
\end{tabular}

1 Fossil collection made by Harold I. Saunders, Paleontology and Stratigraphy Branch, U. S. Geological Survey.

11.75

16.95

17.97

21.89 
Fort Douglas - Continued

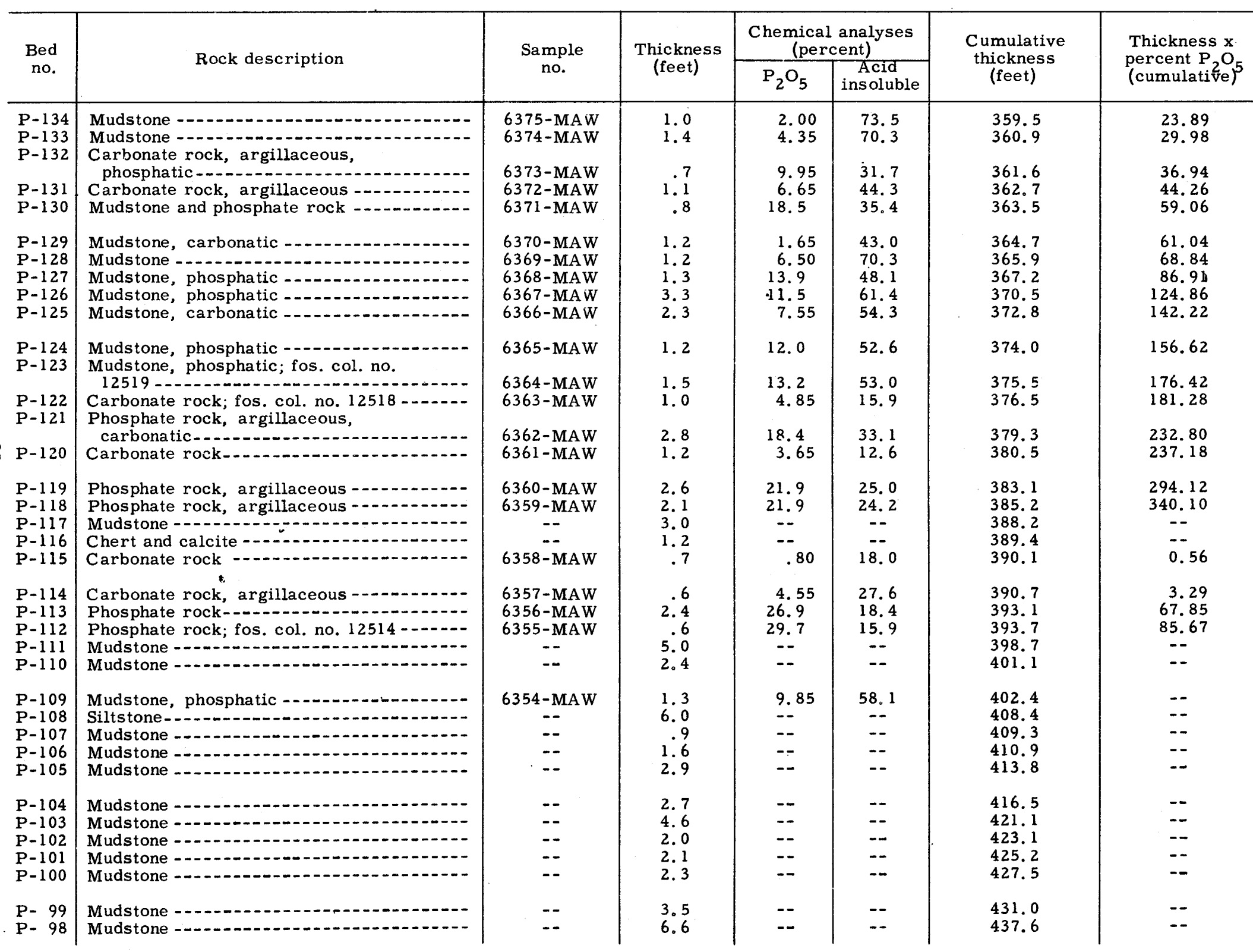


P- 97 Mudstone, cherty

P- 96 Mudstone - 95 Mudstone

P. 94 Siltstone

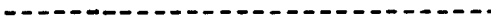

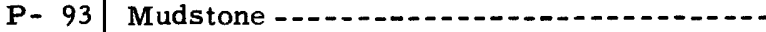

P- 92 Siltstone

P- 91 Mudstone, phosphatic

P- 90 Siltstone; fos. col. no, 12513

P- 89 Chert

P- 88 Mudstone -

P- 87 Carbonate rock-

P- 85 Carbonate rock

85 Chert -..--.--

P- 83

Mudstone - -

P- 82 Mudstone, carbonatic -

$P-81$

Muds tone, carbonatic -

Chert -

P. 79 Chert, argillaceous

P- 78

P- 77

Mudstone, calcareous

Chert, argillaceous -

Mudstone, calcareous and chert; fos.

col. no. 12512

Mudstone, carbonatic -

P- 75

Mudstone, carbonatic and chert

$P-73$

Chert, argillaceous--0.-

P- 72

Carbonate rock, argillaceous; fos.

P- 71

col. no. $12511-$ Chert

P- 70 Mudstone, carbonatic -

P- 69 Mudstone, carbonatic -

P- 68

Mudstone, carbonatic and chert

P- 67

Mudstone, cherty, carbonatic -

P- 66 Limestone -...- 65

P- 65 Mudstone, carbonatic -

P- 64

Mudstone, carbonatic and chert

Mudstone -

P- 62 Mudstone, carbonatic -

P- 61 Mudstone, carbonatic, phosphatic -...-..--

P-60 Mudstone, carbonatic and chert

P- 59

Mudstone, carbonatic

Carbonate rock; fos. col. no. 12510 -...-

P- 57

P- 56

Mudstone, carbonatic

\begin{tabular}{|c|c|c|c|}
\hline$-\infty$ & -- & 438.2 & -- \\
\hline-- & -- & 440.3 & -- \\
\hline- & -- & 443.2 & -- \\
\hline-- & $=-$ & $\begin{array}{l}445.5 \\
446.9\end{array}$ & - \\
\hline & & 170.1 & - \\
\hline 14.3 & ${ }^{-\infty}$ & 447.6 & -- \\
\hline $\begin{array}{c}14.3 \\
--\end{array}$ & 53.1 & 448.0 & -- \\
\hline-- & -- & $\begin{array}{l}451.5 \\
453.7\end{array}$ & -- \\
\hline 4.05 & 78.6 & 454.2 & -- \\
\hline-- & -- & 454.9 & -- \\
\hline-- & - & 456.6 & -- \\
\hline-- & $-\infty$ & 457.0 & $\cdots$ \\
\hline-- & $-\overline{-}$ & $\begin{array}{l}458.1 \\
461.7\end{array}$ & $=$ \\
\hline & & & \\
\hline $\begin{array}{l}1.75 \\
-\end{array}$ & 52.6 & $\begin{array}{l}462.5 \\
467\end{array}$ & -- \\
\hline- & - & $\begin{array}{l}467.5 \\
468.5\end{array}$ & -- \\
\hline-- & - & 470.2 & -- \\
\hline-- & -- & 473.4 & $-\infty$ \\
\hline$-\infty$ & -- & 479.5 & $-\infty$ \\
\hline-- & -- & 481.1 & -- \\
\hline 4.10 & 63.3 & 482.2 & -- \\
\hline-- & -- & 483. 8 & -- \\
\hline-- & -- & 485.1 & -- \\
\hline $\begin{array}{l}5.35 \\
3.95\end{array}$ & $\begin{array}{l}35.3 \\
70.8\end{array}$ & $\begin{array}{l}485.9 \\
486.7\end{array}$ & $\begin{array}{l}4.28 \\
3.16\end{array}$ \\
\hline-- & -- & 489.0 & -- \\
\hline- & -- & 490.1 & -- \\
\hline-- & $\cdots$ & 494.4 & -- \\
\hline-- & -- & 495.5 & -- \\
\hline$-\infty$ & $=$ & 497.9 & -- \\
\hline 4.65 & 65.6 & 498.8 & -- \\
\hline-- & -- & $\begin{array}{l}500.6 \\
501\end{array}$ & 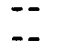 \\
\hline-- & - & 501.9 & $\ldots$ \\
\hline- & -- & 503.7 & -- \\
\hline 8.85 & 44.8 & 504.1 & -- \\
\hline- & $=$ & $\begin{array}{l}505.4 \\
506.8\end{array}$ & -- \\
\hline- & - & 508.6 & - \\
\hline 7.25 & 40.9 & 509.1 & -- \\
\hline-- & -- & 510.4 & -- \\
\hline
\end{tabular}


Fort Douglas - Continued

\begin{tabular}{|c|c|c|c|c|c|c|c|}
\hline \multirow{2}{*}{$\begin{array}{c}\text { Bed } \\
\text { no. }\end{array}$} & \multirow{2}{*}{ Rock description } & \multirow{2}{*}{$\begin{array}{l}\text { Sample } \\
\text { no. }\end{array}$} & \multirow{2}{*}{$\begin{array}{l}\text { Thickness } \\
\text { (feet) }\end{array}$} & \multicolumn{2}{|c|}{$\begin{array}{c}\text { Chemical analyses } \\
\text { (percent) }\end{array}$} & \multirow{2}{*}{$\begin{array}{l}\text { Cumulative } \\
\text { thickstess } \\
\text { (feet) }\end{array}$} & \multirow{2}{*}{$\begin{array}{l}\text { Thickness } \mathrm{x} \\
\text { percent } \mathrm{P}_{2} \mathrm{O}_{5} \\
\text { (cumulative) }\end{array}$} \\
\hline & & & & $\mathrm{P}_{2} \mathrm{O}_{5}$ & $\begin{array}{l}\text { Acid } \\
\text { ins oluble }\end{array}$ & & \\
\hline $\begin{array}{l}P-55 \\
P-54 \\
P-53 \\
P-52 \\
P-51\end{array}$ & $\begin{array}{l}\text { Mudstone } \\
\text { Mudstone } \\
\text { Mudstone, carbonatic; fos. col. no. } 12509 \\
\text { Mudstone } \\
\text { Mudstone }\end{array}$ & $\begin{array}{l}-- \\
-- \\
-- \\
-- \\
--\end{array}$ & $\begin{array}{l}3.3 \\
4.2 \\
4.1 \\
3.5 \\
4.7\end{array}$ & $\begin{array}{l}-- \\
-- \\
-- \\
--\end{array}$ & $\begin{array}{l}-- \\
-- \\
-- \\
--\end{array}$ & $\begin{array}{l}513.7 \\
517.9 \\
522.0 \\
525.5 \\
530.2\end{array}$ & $\begin{array}{l}-- \\
-- \\
-- \\
--\end{array}$ \\
\hline $\begin{array}{l}P-50 \\
P-49 \\
P-48 \\
P-47 \\
P-46\end{array}$ & $\begin{array}{l}\text { Mudstone } \\
\text { Mudstone, carbonatic } \\
\text { Carbonate rock; fos. col. no. } 12508 \\
\text { Mudstone, carbonatic; fos. col. no. } 12507 \\
\text { Covered interval }\end{array}$ & $\begin{array}{l}-- \\
-- \\
-- \\
--\end{array}$ & $\begin{array}{r}1.2 \\
3.6 \\
1.5 \\
2.9 \\
1+.8\end{array}$ & $\begin{array}{l}-- \\
-- \\
-- \\
--\end{array}$ & $\begin{array}{l}-- \\
-- \\
-- \\
-- \\
--\end{array}$ & $\begin{array}{l}531.4 \\
535.0 \\
536.5 \\
539.4 \\
551.2\end{array}$ & $\begin{array}{l}-- \\
-- \\
-- \\
--\end{array}$ \\
\hline $\begin{array}{l}P-45 \\
P-44 \\
P-43 \\
P-42\end{array}$ & $\begin{array}{l}\text { Carbonate rock } \\
\text { Mudstone, carbonatic; fos. col. no. } 12506 \\
\text { Carbonate rock } \\
\text { Carbonate rock, argillaceous; fos. col. }\end{array}$ & $\begin{array}{l}-- \\
-- \\
--\end{array}$ & $\begin{array}{l}1.9 \\
4.6 \\
1.8\end{array}$ & -- & $\begin{array}{l}-- \\
--\end{array}$ & $\begin{array}{l}553.1 \\
557.7 \\
559.5\end{array}$ & -- \\
\hline$P-41$ & $\begin{array}{l}\text { no. } 12505 \\
\text { Mudstone, cherty; fos. col. no. } 12505\end{array}$ & -- & $\begin{array}{l}3.6 \\
4.5\end{array}$ & -- & -- & $\begin{array}{l}563.1 \\
567.6\end{array}$ & -- \\
\hline $\begin{array}{l}P-40 \\
P-39 \\
P-38 \\
P-37 \\
P-36\end{array}$ & $\begin{array}{l}\text { Mudstone, cherty; fos. col. no. } 12504 \\
\text { Mudstone, carbonatic } \\
\text { Mudstone, carbonatic } \\
\text { Mudstone, carbonatic } \\
\text { Mudstone, carbonatic; fos. col. no. } 12503\end{array}$ & $\begin{array}{l}-- \\
-- \\
-- \\
--\end{array}$ & $\begin{array}{l}5.5 \\
5.3 \\
2.9 \\
1.2 \\
2.9\end{array}$ & $\begin{array}{l}-- \\
-- \\
-- \\
--\end{array}$ & $\begin{array}{l}-- \\
-- \\
-- \\
--\end{array}$ & $\begin{array}{l}573.1 \\
578.4 \\
581.3 \\
582.5 \\
585.4\end{array}$ & $\begin{array}{l}-- \\
-- \\
-- \\
--\end{array}$ \\
\hline $\begin{array}{l}P-35 \\
P-34 \\
P-33 \\
P-32 \\
P-31\end{array}$ & $\begin{array}{l}\text { Mudstone, carbonatic; fos. col. no. } 12502 \\
\text { Mudstone } \\
\text { Carbonate rock, argillaceous } \\
\text { Carbonate rock, cherty } \\
\text { Carbonate rock, argillaceous }\end{array}$ & $\begin{array}{l}-- \\
-- \\
-- \\
--\end{array}$ & $\begin{array}{l}5.9 \\
2.3 \\
2.6 \\
2.4 \\
4.1\end{array}$ & $\begin{array}{l}-- \\
-- \\
-- \\
-\infty\end{array}$ & $\begin{array}{l}-- \\
-- \\
-- \\
--\end{array}$ & $\begin{array}{l}591.3 \\
593.6 \\
596.2 \\
598.6 \\
602.7\end{array}$ & $\begin{array}{l}-- \\
-- \\
-- \\
--\end{array}$ \\
\hline $\begin{array}{l}P-30 \\
P-29 \\
P-28 \\
P-27 \\
P-26\end{array}$ & $\begin{array}{l}\text { Mudstone } \\
\text { Mudstone } \\
\text { Mudstone } \\
\text { Mudstone } \\
\text { Mudstone, carbonatic }\end{array}$ & $\begin{array}{l}-- \\
-- \\
-- \\
--\end{array}$ & $\begin{array}{r}3.7 \\
11.0 \\
1.6 \\
2.0 \\
2.9\end{array}$ & $\begin{array}{l}-- \\
-- \\
-- \\
--\end{array}$ & $\begin{array}{l}-- \\
-- \\
-- \\
--\end{array}$ & $\begin{array}{l}606.4 \\
617.4 \\
619.0 \\
621.0 \\
623.9\end{array}$ & $\begin{array}{l}-- \\
-- \\
-- \\
--\end{array}$ \\
\hline $\begin{array}{l}P-25 \\
P-24 \\
P-23 \\
P-22 \\
P-21\end{array}$ & $\begin{array}{l}\text { Mudstone, carbonatic } \\
\text { Mudstone, carbonatic } \\
\text { Mudstone, carbonatic } \\
\text { Mudstone, carbonatic } \\
\text { Mudstone, carbonatic }\end{array}$ & $\begin{array}{l}-- \\
-- \\
-- \\
--\end{array}$ & $\begin{array}{l}3.8 \\
5.2 \\
4.8 \\
.9 \\
5.1\end{array}$ & $\begin{array}{l}-- \\
-- \\
-- \\
--\end{array}$ & $\begin{array}{l}-- \\
-- \\
-- \\
-- \\
--\end{array}$ & $\begin{array}{l}627.7 \\
632.9 \\
637.7 \\
638.6 \\
643.7\end{array}$ & $\begin{array}{l}-- \\
-- \\
-- \\
--\end{array}$ \\
\hline $\begin{array}{l}P-20 \\
P-19 \\
P-18 \\
P-17\end{array}$ & $\begin{array}{l}\text { Mudstone, carbonatic } \\
\text { Carbonate rock, argillaceous } \\
\text { Carbonate rock, argillaceous } \\
\text { Phosphate rock, carbonatic }\end{array}$ & $\begin{array}{l}\text { 6343- TMC } \\
6342-\text { TMC } \\
6341-\text { TMC }\end{array}$ & $\begin{array}{l}2.3 \\
1.4 \\
1.1 \\
1.5\end{array}$ & $\begin{array}{l}6.25 \\
7.75 \\
21.1\end{array}$ & $\begin{array}{l}-\infty \\
23.1 \\
24.1 \\
16.1\end{array}$ & $\begin{array}{l}646.0 \\
647.4 \\
648.5 \\
650.0\end{array}$ & $\begin{array}{r}-2.75 \\
17.28 \\
48.92\end{array}$ \\
\hline
\end{tabular}




\begin{tabular}{|c|c|c|c|c|c|c|c|}
\hline$P-16$ & Carbonate rock, argillaceous & $6340-\mathrm{TMC}$ & 2.7 & 7.60 & 31.2 & 652.7 & 69.44 \\
\hline$P-15$ & Mudstone, carbonatic & -- & 2.6 & -- & -- & 655.3 & -- \\
\hline $\mathrm{P}-14$ & Mudstone, carbonatic & -- & 5.3 & -- & -- & 660.6 & -- \\
\hline $\begin{array}{l}P-13 \\
P-12\end{array}$ & $\begin{array}{l}\text { Mudstone, carbonatic } \\
\text { Mudstone, carbonatic; fos. col. no. }\end{array}$ & -- & 1.3 & -- & -- & 661.9 & -- \\
\hline & $12517 \ldots$ & -- & 1.1 & -- & - & 663.0 & $-\infty$ \\
\hline$P-11$ & Mudstone, carbonatic & -- & 4.6 & -- & - & 667.6 & -- \\
\hline $\begin{array}{l}P-10 \\
P-9\end{array}$ & Mudstone, carbonatic & $6339-\overline{T M C}$ & 2.2 & $\overline{0.1}$ & 23.7 & $\begin{array}{l}669.8 \\
670.2\end{array}$ & -- \\
\hline $\begin{array}{l}P-9 \\
P-8\end{array}$ & $\begin{array}{l}\text { Carbonate rock, argillaceous, phos phatic -- } \\
\text { Mudstone, carbonatic }\end{array}$ & $\begin{array}{c}0339-1 \mathrm{MC} \\
--\end{array}$ & 2.6 & 10.1 & 23. & $\begin{array}{l}670.2 \\
672.8\end{array}$ & -- \\
\hline$P-7$ & Mudstone, carbonatic & -- & 2.1 & -- & -- & 674.9 & -- \\
\hline P- 6 & Mudstone & -- & .9 & -- & -- & 675.8 & -- \\
\hline$P-5$ & Mudstone, carbonatic & -- & 3.2 & -- & -- & 679.0 & -- \\
\hline$P-4$ & Muds tone & -- & 1.3 & -- & -- & 680.3 & -- \\
\hline P- 3 & Mudstone, carbonatic & - & $\cdot 7$ & -- & -- & 681.0 & -- \\
\hline P- 2 & Covered interval & -- & .6 & - & - & 681.6 & - \\
\hline P- 1 & Phosphate rock, carbonatic & -- & 1.2 & -- & -- & 682.8 & -- \\
\hline \multicolumn{8}{|c|}{ Lower member of Park City formation (?) } \\
\hline $\begin{array}{l}L-19 \\
L-18 \\
L-17 \\
L=16 \\
L-15\end{array}$ & $\begin{array}{l}\text { Carbonate rock } \\
\text { Carbonate rock } \\
\text { Sandstone } \\
\text { Siltstone } \\
\text { Siltstone }\end{array}$ & $\begin{array}{l}-- \\
-- \\
-- \\
--\end{array}$ & $\begin{array}{l}60.0 \\
28.0 \\
14.0 \\
16.0 \\
31.0\end{array}$ & $\begin{array}{l}-- \\
-- \\
-- \\
--\end{array}$ & $\begin{array}{l}-- \\
-- \\
-- \\
-- \\
--\end{array}$ & $\begin{array}{r}60.0 \\
88.0 \\
102.0 \\
118.0 \\
149.0\end{array}$ & $\begin{array}{l}-- \\
-- \\
-- \\
--\end{array}$ \\
\hline $\begin{array}{l}L-14 \\
L-13 \\
L-12 \\
L-11 \\
L-10\end{array}$ & $\begin{array}{l}\text { Sandstone } \\
\text { Sandstone } \\
\text { Carbonate rock } \\
\text { Covered interval }\end{array}$ & $\begin{array}{l}-- \\
-- \\
-- \\
--\end{array}$ & $\begin{array}{r}54.0 \\
30.0 \\
7.0 \\
(?) \\
52.0\end{array}$ & $\begin{array}{l}-- \\
-- \\
-- \\
--\end{array}$ & $\begin{array}{l}-- \\
-- \\
-- \\
--\end{array}$ & $\begin{array}{l}203.0 \\
233.0 \\
240.0 \\
-52.0\end{array}$ & $\begin{array}{l}-- \\
-- \\
-- \\
--\end{array}$ \\
\hline $\begin{array}{l}L-9 \\
L-8 \\
L-7 \\
L=6 \\
L-5\end{array}$ & $\begin{array}{l}\text { Carbonate rock } \\
\text { Sandstone } \\
\text { Carbonate rock } \\
\text { Sandstone- } \\
\text { Sandstone }\end{array}$ & $\begin{array}{l}-- \\
-- \\
-- \\
-- \\
--\end{array}$ & $\begin{array}{r}1.5 \\
6.5 \\
7.0 \\
18.0 \\
38.0\end{array}$ & $\begin{array}{l}-- \\
-- \\
-- \\
-- \\
--\end{array}$ & $\begin{array}{l}-- \\
-- \\
-- \\
-- \\
--\end{array}$ & $\begin{array}{r}53.5 \\
60.0 \\
67.0 \\
85.0 \\
123.0\end{array}$ & $\begin{array}{l}-- \\
-- \\
-- \\
-- \\
--\end{array}$ \\
\hline$L-4$ & Sands tone- & -- & 60.0 & $\ddot{--}$ & -- & 183.0 & -- \\
\hline $\begin{array}{l}L-3 \\
L-2\end{array}$ & Sands tone and sandy carbonate rock & -- & $\begin{array}{l}30.0 \\
25.0\end{array}$ & -- & -- & $\begin{array}{l}213.0 \\
238.0\end{array}$ & -- \\
\hline $\begin{array}{l}L-2 \\
L-1\end{array}$ & $\begin{array}{l}\text { Carbonate rock } \\
\text { Sandstone }\end{array}$ & -- & $\begin{array}{r}25.0 \\
7.0\end{array}$ & -- & -- & $\begin{array}{r}238.0 \\
* * 245.0\end{array}$ & $\ddot{--}$ \\
\hline \multicolumn{8}{|c|}{ Weber formation } \\
\hline $\mathrm{C} w-1$ & Sands tone & -- & 100 & -- & -- & 100 & -- \\
\hline
\end{tabular}

* Cumulative data incomplete because of missing information. Computations start from zero after interruption.

** Note incompleteness of cumulative data. 


\section{Sols Canyon, Utah, lot 1348}

Park City formation measured and sampled in outcrop and hand trench on east side of Sols Canyon, NW $\frac{1}{4}$ SW $\frac{1}{4}$ sec. 2, T. 2 N., R. 18 E., Daggett County, Utah. Beds strike N. $80^{\circ}$ E. and dip $38^{\circ}$ N. Section measured and sampled by R. A. Smart, T. M. Cheney, and M. A. Warner in June 1951. Samples analyzed by Trace Elements Section laboratory, Denver, Colo.

\begin{tabular}{c|c|c|c|c|c|c|}
\hline \multirow{2}{*}{$\begin{array}{c}\text { Bed } \\
\text { no. }\end{array}$} & Rock description & \multirow{2}{*}{$\begin{array}{c}\text { Sample } \\
\text { no. }\end{array}$} & $\begin{array}{c}\text { Thickness } \\
\text { (feet) }\end{array}$ & $\begin{array}{c}\text { Chemical analyses } \\
\text { (percent) }\end{array}$ & $\begin{array}{c}\text { Cumulative } \\
\text { thickness } \\
\text { (feet) }\end{array}$ & $\begin{array}{c}\text { Thickness } \mathbf{x} \\
\text { percent } P_{2} \mathbf{O}_{5} \\
(\text { cumulative) }\end{array}$ \\
\hline
\end{tabular}

Upper member of Park City formation - top not exposed

\begin{tabular}{l|l|l|l}
\hline U- 7 & Carbonate rock, cherty & -- & 9.1 \\
U- 6 & Carbonate rock & -1.4 \\
U- 5 & Covered interval & - & 4.0 \\
U- 4 & Carbonate rock & -1.8 \\
U- 3 & Covered interval & -- & 3.8 \\
U- 2 & Carbonate rock & & 1.6 \\
U- 1 & Covered interval & -- & 3.0
\end{tabular}

Phosphatic shale member of Park City formation

w P-47 Siltstone, carbonatic$\mathrm{P}-46$ Mudstone and chert -

$\mathrm{P}-45$ Mudstone, carbonatic

(1)

P-42 Mudstone -

P-41 Mudstone, carbonatic $\quad$ Mudstone, carbonatic

$\mathrm{P}-40$

Mudstone, carbonatic

P-38 Weathered zone

P-37 Carbonate rock, argillaceous, phosphatic --

P-36 Carbonate rock, phosphatic -

P-36 Carbonate rock, phosphatic- Carbonate rock-

P-35 Carbonate rock
P-34 Mudstone, carbonatic, and phosphatic,

P-33 Carbonate rock, argillaceous, phosphatic -

P-32 Phosphate rock and phosphatic, carbonatic

P-31 Mudstone, carbonatic

P-31 Mudstone, carbonatic
P-30 Mudstone, carbonatic, and carbonatic

phosphate rock-...

P-29 Mudstone, carbonatic -

P-28

Mudstone, silty -

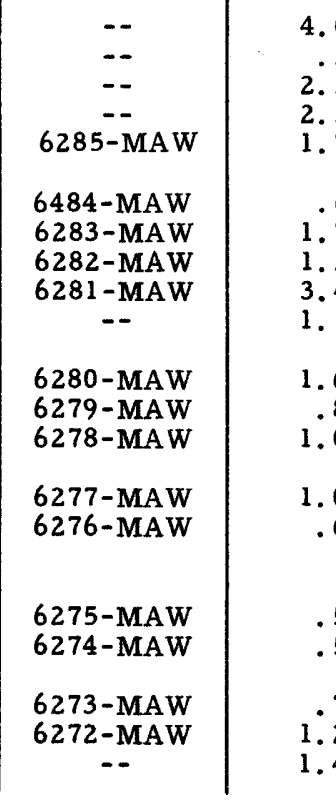

9.1
5.4
4.0
1.8
3.8
1.6
3.0

\begin{tabular}{l|l|l|}
-- & -- \\
-- & -- \\
-- & - \\
-- & -- \\
-- & -- \\
-- & -- \\
\hline- & & \\
\hline
\end{tabular}

\begin{tabular}{r|l}
9.1 & -- \\
14.5 & -- \\
18.5 & -- \\
20.3 & -- \\
24.1 & -- \\
25.7 & -- \\
28.7 & -- \\
\hline
\end{tabular}

--

$-$

3.60

$--$

$--$

2. 10

7. 25

2. 75

--

39.8

-.

58. 7

44.5
60.3

--

11.7

14.2

35.6

35.6
12.7

$7.45 \quad 10.3$

10.7
9.00

27.9
27.4

4.0

7. 5

7.0
9.5

11.2

11.8

13.5

14. 7

18. 1

19.2

20.8

21.6

22.6

23.6

24.2

$-$

.6

.5
.5

15.8

7.25

32.5

43.5

24. 7

25.2

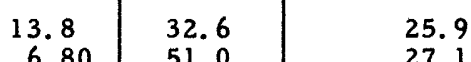

27.1

28.5
65.16

$-$

$-$

$\overline{--}$

$--$

**3. 57

\begin{tabular}{l}
12.27 \\
21.62 \\
\hline
\end{tabular}

21.62

*18. 72

30.08

37.53

48. 23

53.63

61.53

74.82

82.98 


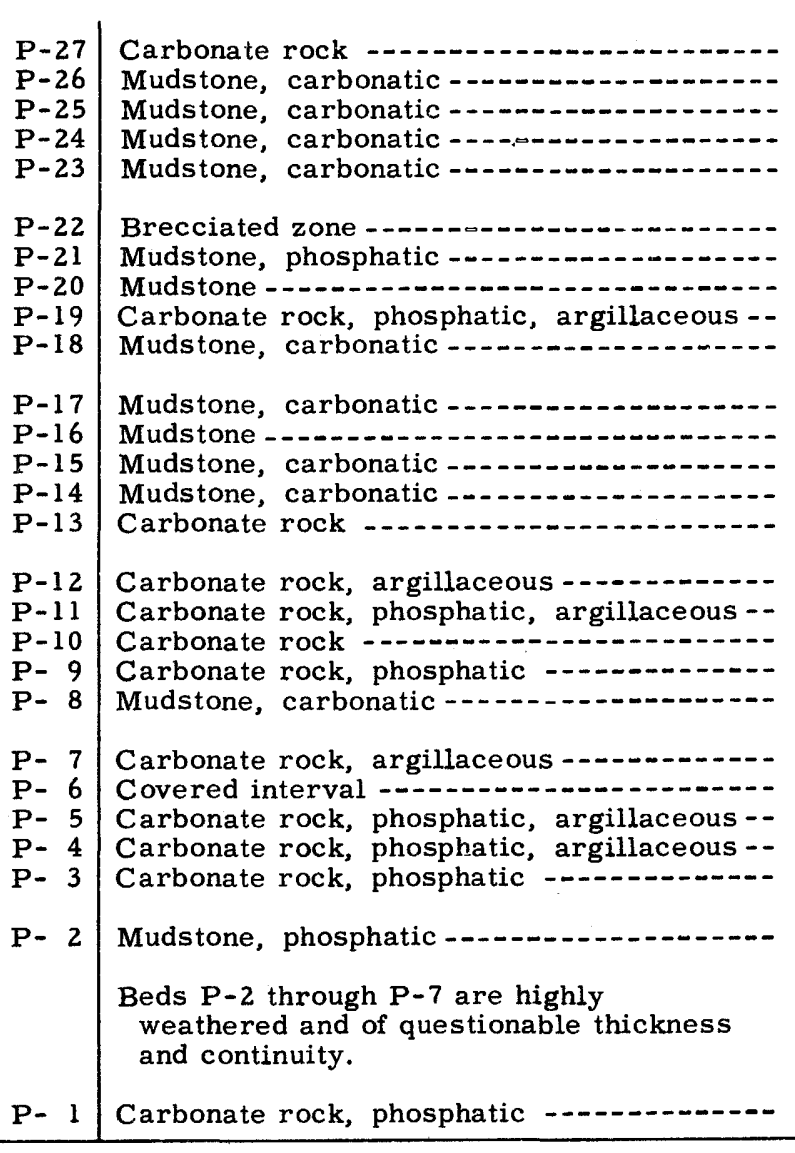

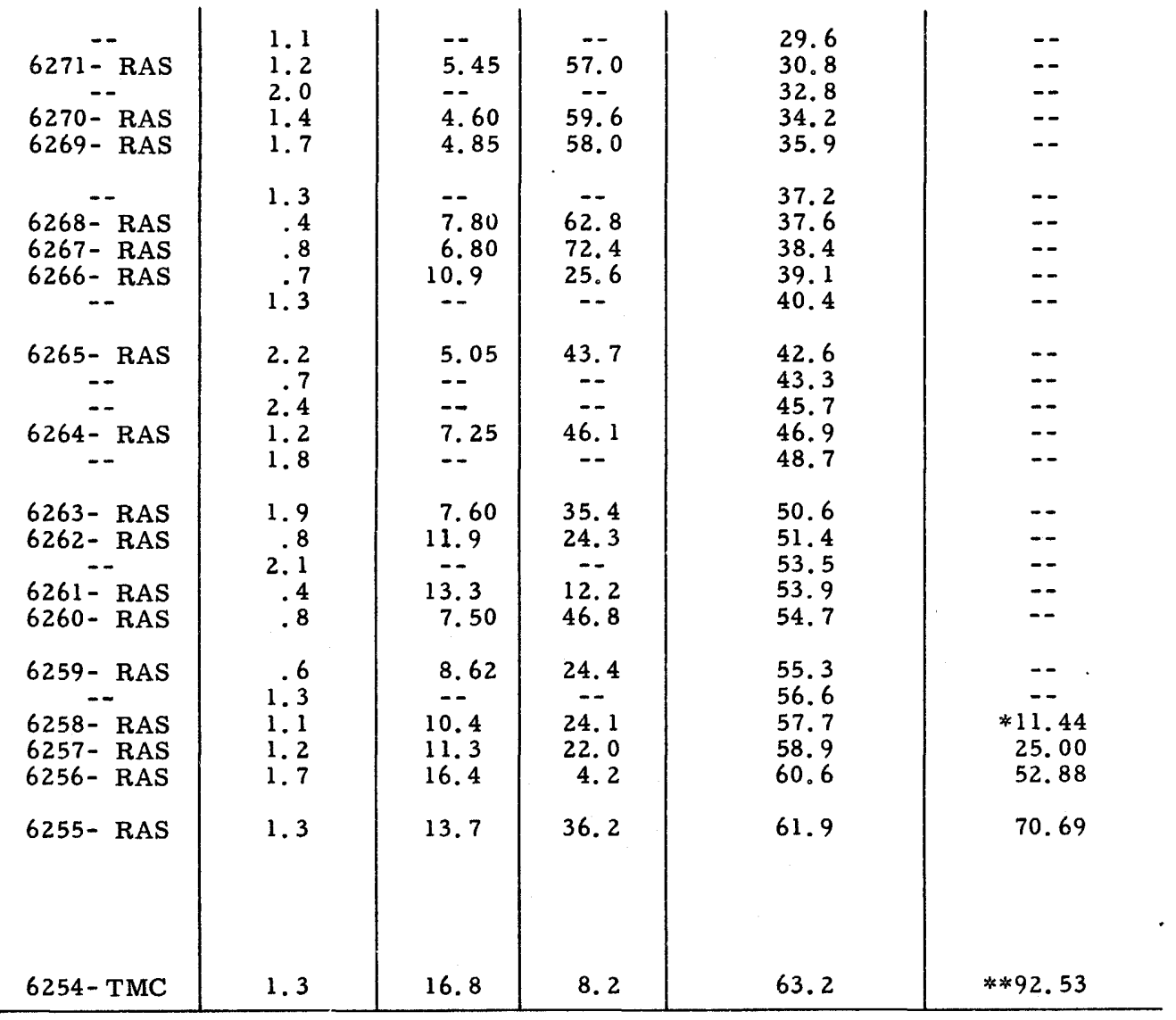

Lower member of Park City formation

\begin{tabular}{l|l}
\hline L-17 & Sandstone, carbonatic \\
L-16 & Mudstone, carbonatic \\
L-15 & Sandstone, carbonatic \\
L-14 & Sandstone, carbonatic \\
L-13 & Sandstone, carbonatic \\
L-12 & Sandstone, argillaceous \\
L-11 & Sandstone, carbonatic \\
L-10 & Silts tone, carbonatic \\
L-9 & Sandstone, carbonatic
\end{tabular}

\begin{tabular}{l|r}
-- & 17.6 \\
-- & 3.4 \\
-- & 6.4 \\
-- & 15.0 \\
-- & 10.5 \\
-- & 3.5 \\
-- & 10.5 \\
-- & 2.5 \\
-- & 8.5
\end{tabular}

* Cumulative data incomplete because of missing information. Computations start from zero after interruption.

* Note incompleteness of cumulative data. 
Sols Canyon-Continued

\begin{tabular}{|c|c|c|c|c|c|c|c|}
\hline \multirow{2}{*}{$\begin{array}{c}\text { Bed } \\
\text { no. }\end{array}$} & \multirow{2}{*}{ Rock description } & \multirow{2}{*}{$\begin{array}{c}\text { Sample } \\
\text { no. }\end{array}$} & \multirow{2}{*}{$\begin{array}{c}\text { Thickness } \\
\text { (feet) }\end{array}$} & \multicolumn{2}{|c|}{$\begin{array}{c}\text { Chemical analyses } \\
\text { (percent) }\end{array}$} & \multirow{2}{*}{$\begin{array}{l}\text { Cumulative } \\
\text { thickness } \\
\text { (feet) }\end{array}$} & \multirow{2}{*}{$\begin{array}{l}\text { Thickness } \mathrm{x} \\
\text { percent } \mathrm{P}_{2} \mathrm{O}_{5} \\
\text { (cumulative) }\end{array}$} \\
\hline & & & & $\mathrm{P}_{2} \mathrm{O}_{5}$ & $\begin{array}{c}\text { Acid } \\
\text { insoluble }\end{array}$ & & \\
\hline $\begin{array}{l}L-8 \\
L-7 \\
L-6 \\
L-5 \\
L-4\end{array}$ & $\begin{array}{l}\text { Siltstone, carbonatic } \\
\text { Sandstone, carbonatic } \\
\text { Covered interval } \\
\text { Sandstone, carbonatic } \\
\text { Sandstone, carbonatic }\end{array}$ & $\begin{array}{l}-- \\
-- \\
-- \\
--\end{array}$ & $\begin{array}{r}5.0 \\
23.0 \\
3.5 \\
6.0 \\
23.0\end{array}$ & $\begin{array}{l}-- \\
-- \\
-- \\
--\end{array}$ & $\begin{array}{l}-- \\
-- \\
-- \\
--\end{array}$ & $\begin{array}{r}82.9 \\
105.9 \\
109.4 \\
115.4 \\
138.4\end{array}$ & $\begin{array}{l}-- \\
-- \\
-- \\
--\end{array}$ \\
\hline $\begin{array}{l}L-3 \\
L-2 \\
L-1\end{array}$ & $\begin{array}{l}\text { Covered interval } \\
\text { Sandstone } \\
\text { Covered interval }\end{array}$ & $\begin{array}{l}-- \\
--\end{array}$ & $\begin{array}{l}20.0 \\
25.0 \\
23.0\end{array}$ & $\begin{array}{l}-- \\
--\end{array}$ & -- & $\begin{array}{l}158.4 \\
183.4 \\
206.4\end{array}$ & -- \\
\hline \multicolumn{8}{|c|}{ Weber formation - upper part only } \\
\hline $\mathrm{Cw}-1$ & Sandstone, carbonatic & -- & 205.0 & -- & -- & 205.0 & $-\infty$ \\
\hline
\end{tabular}


Park City formation measured and sampled in outcrop and bulldozer trenches in Willow Creek Canyon, NW $\frac{1}{4}$ sec. 14, T. $2 \mathrm{~S} ., \mathrm{R} .12 \mathrm{~W}$. , Wasatch County, Utah. Beds strike N. $30^{\circ} \mathrm{W}$. and are overturned toward the west; dips range from $35^{\circ} \mathrm{W}$. to near vertical. Section measured and sampled by R. A. Smart, T. M. Cheney, and R. G. Waring in September 1951. Samples analyzed by U. S. Bureau of Mines laboratory, Albany, Oreg.

\begin{tabular}{c|c|c|c|c|c|c|c}
\hline $\begin{array}{c}\text { Bed } \\
\text { no. }\end{array}$ & Rock description & Sample & \multirow{2}{*}{$\begin{array}{c}\text { Thickness } \\
\text { (feet) }\end{array}$} & $\begin{array}{c}\text { Chemical analyses } \\
\text { (percent) }\end{array}$ & $\begin{array}{c}\text { Cumulative } \\
\text { thickness } \\
\text { (feet) }\end{array}$ & $\begin{array}{c}\text { Thickness } \mathbf{x} \\
\text { percent } P_{2} \mathrm{O}_{5} \\
\text { (cumulative) }\end{array}$ \\
\hline
\end{tabular}

Woodside formation- not measured

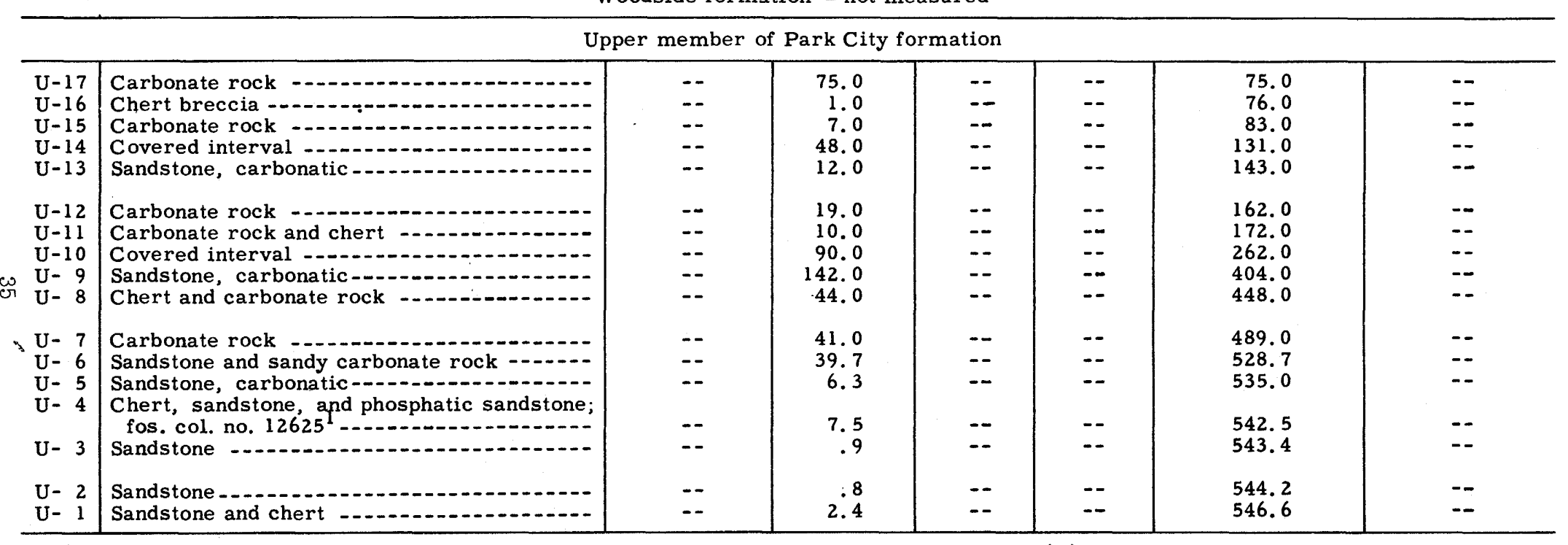

Phosphatic shale member of Park City formation (?)

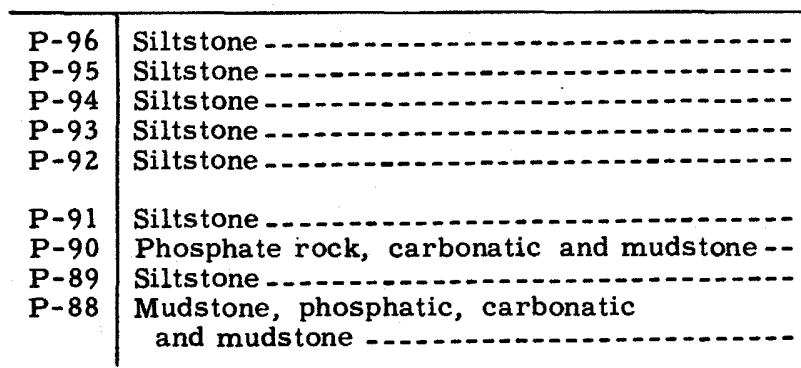

\begin{tabular}{c|r}
-- & 4.5 \\
-- & 6.3 \\
-- & 2.1 \\
-- & 5.5 \\
& 14.5 \\
6618-RGW & 1.2 \\
6617-RGW & 1.1 \\
6616-RGW & 2.5 \\
6615-RGW & 1.0
\end{tabular}

\begin{tabular}{l|}
4.5 \\
6.3 \\
2.1 \\
5.5 \\
4.5 \\
1.2 \\
1.1 \\
2.5 \\
1.0
\end{tabular}

\begin{tabular}{|c|c|}
-- & -- \\
-- & -- \\
-- & -- \\
-- & -- \\
3.3 & 67.5 \\
16.1 & 34.4 \\
2.8 & 66.0 \\
4.4 & 62.5
\end{tabular}

4.5
10.8
12.9
18.4
32.9
34.1
35.2
37.7
38.7

$--$

$-$

$--$

* Fossil collection made by J. E. Smedley, Paleontology and Stratigraphy Branch, U. S. Geological Survey.

* Note incompleteness of cumulative data because of missing information. 
Strawberry Valley - Continued

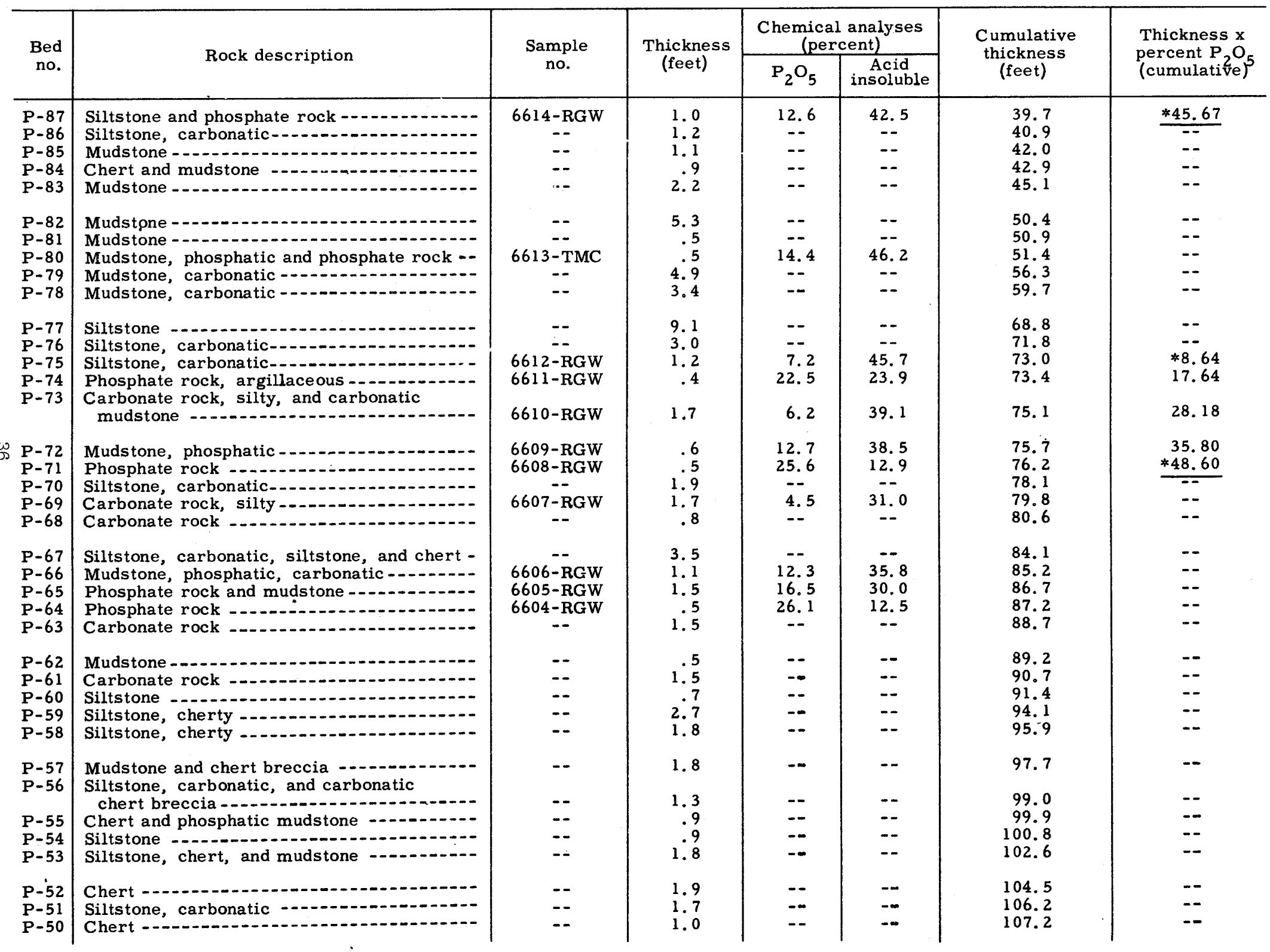




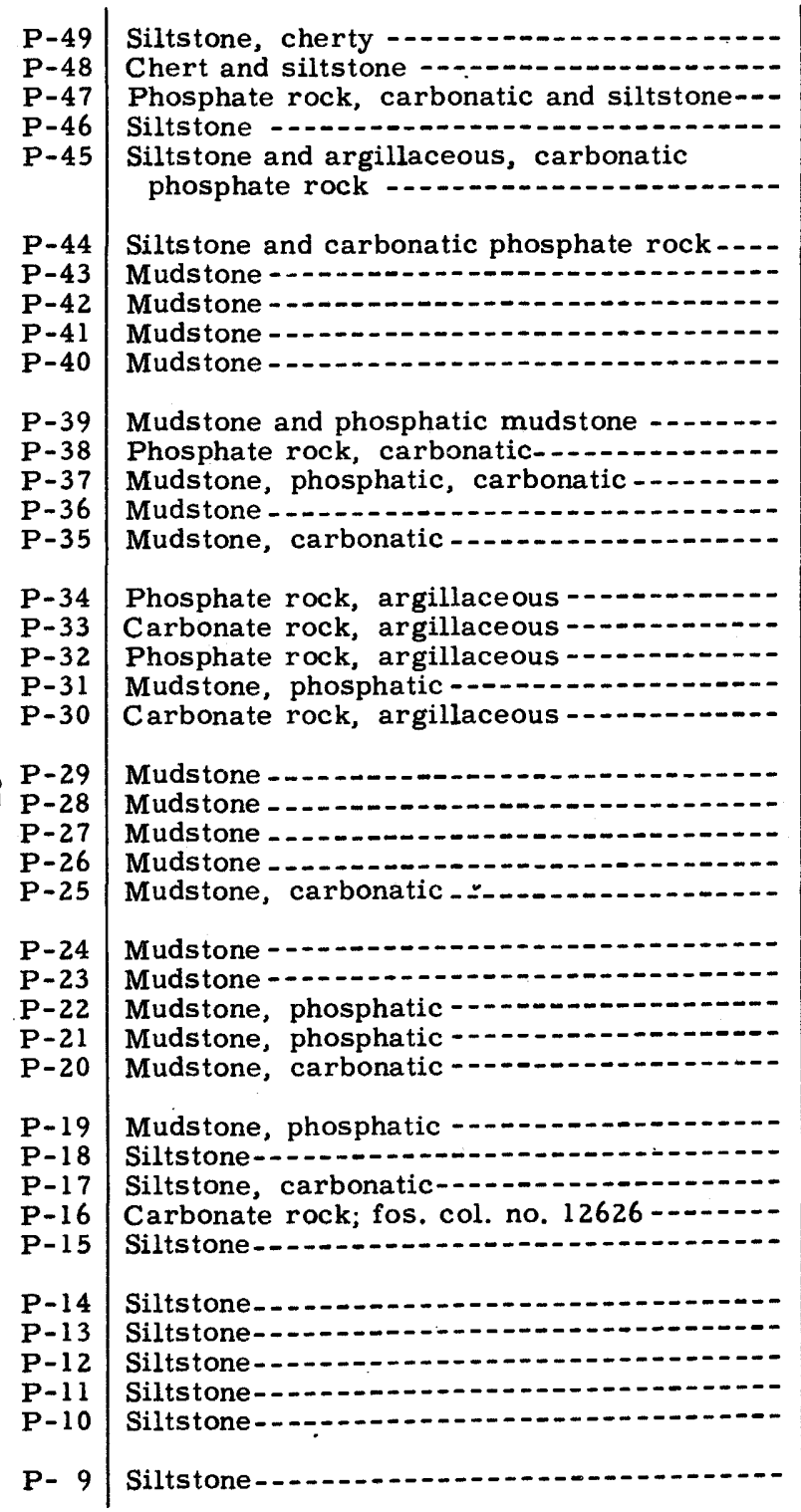

\begin{tabular}{|c|c|}
\hline-- & 2.5 \\
\hline 6603-RGW & $\begin{array}{r}1.6 \\
.4\end{array}$ \\
\hline-- & 2.5 \\
\hline-- & 1.0 \\
\hline.- & .6 \\
\hline-- & 3.9 \\
\hline 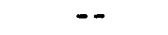 & 1.1 \\
\hline- & 1.1 \\
\hline- & 1.0 \\
\hline-- & 1.1 \\
\hline 6602-RAS & .6 \\
\hline 6601 - RAS & $\begin{array}{l}1.7 \\
1.8\end{array}$ \\
\hline-- & 1.5 \\
\hline 6600-RAS & .5 \\
\hline 6599- RAS & 1.6 \\
\hline $\begin{array}{l}\text { 6598-RAS } \\
6597-\text { RAS }\end{array}$ & 1.9 \\
\hline 6596- RAS & 1.1 \\
\hline-- & 1.5 \\
\hline-- & 2. 1 \\
\hline$\ldots$ & 1.5 \\
\hline-- & 2.5 \\
\hline & \\
\hline-- & 1.1 \\
\hline $6595-\mathrm{RAS}$ & $\begin{array}{r}4.5 \\
9\end{array}$ \\
\hline 6594-RAS & .75 \\
\hline 6593-RAS & .8 \\
\hline 6592- RAS & .5 \\
\hline-- & 1.2 \\
\hline-- & 2.9 \\
\hline-- & 3.0 \\
\hline-- & 2.3 \\
\hline & .7 \\
\hline-8 & 1.5 \\
\hline-- & 2. 7 \\
\hline-- & .8 \\
\hline
\end{tabular}

\begin{tabular}{|c|c|}
\hline-- & -- \\
\hline 17.9 & 32.1 \\
\hline-- & - \\
\hline-- & -- \\
\hline- & - \\
\hline- & - \\
\hline-- & -- \\
\hline-- & -- \\
\hline-- & - \\
\hline 18.5 & 14.6 \\
\hline 8.7 & 36.0 \\
\hline$\overline{-}$ & -- \\
\hline & \\
\hline $\begin{array}{r}23.9 \\
2.4\end{array}$ & $\begin{array}{l}17.3 \\
31.0\end{array}$ \\
\hline 20.7 & 24.9 \\
\hline 12. 7 & 40.5 \\
\hline 4.3 & 36.5 \\
\hline -- & - \\
\hline & - \\
\hline -. & $\ldots$ \\
\hline-- & -- \\
\hline- & - \\
\hline 3.3 & 53.0 \\
\hline 15.7 & 42.4 \\
\hline 2.8 & 63.3 \\
\hline 8.3 & 58.5 \\
\hline & \\
\hline 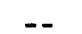 & \\
\hline- & $\cdots$ \\
\hline 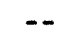 & -- \\
\hline$\because$ & -- \\
\hline & 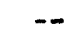 \\
\hline- & - \\
\hline-- & -- \\
\hline
\end{tabular}

\begin{tabular}{l|l}
109.7 & -- \\
111.3 & -- \\
111.7 & -- \\
114.2 & -- \\
115.2 & -- \\
115.8 & -- \\
119.7 & -- \\
120.8 & -- \\
121.9 & -- \\
122.9 & -- \\
124.0 & - \\
124.6 & - \\
126.3 & - \\
128.1 & -- \\
129.6 &
\end{tabular}

130.1

131.7

132.8

133.7
134.8

136.3

138.4

142.4

143.0

144.1

148.6

149.5

150.0

150.8

151.3

152.5

153.5

156.4

159.4

161.7

162.4

163.3

164.8

167.5

168.3

*11. 95

15.79

38.56

49.99

54.72

--

$-$

$-$

$--$

$-$

*7. 47

15.32

17.56

* Note incompleteness of cumulative data because of missing information.

\begin{tabular}{c}
$* 21.71$ \\
\hline-- \\
-- \\
-- \\
-- \\
-- \\
-- \\
-- \\
--
\end{tabular}


Strawberry Valley-Continued

\begin{tabular}{|c|c|c|c|c|c|c|c|}
\hline \multirow{2}{*}{$\begin{array}{c}\text { Bed } \\
\text { no. }\end{array}$} & \multirow{2}{*}{ Rock description } & \multirow{2}{*}{$\begin{array}{l}\text { Sample } \\
\text { no. }\end{array}$} & \multirow{2}{*}{$\begin{array}{c}\text { Thickness } \\
\text { (feet) }\end{array}$} & \multicolumn{2}{|c|}{$\begin{array}{c}\text { Chemical analyses } \\
\text { (percent) }\end{array}$} & \multirow{2}{*}{$\begin{array}{l}\text { Cumulative } \\
\text { thickness } \\
\text { (feet) }\end{array}$} & \multirow{2}{*}{$\begin{array}{l}\text { Thickness } \mathrm{x} \\
\text { percent } \mathrm{P}_{2} \mathrm{O}_{5} \\
\text { (cumulative) }\end{array}$} \\
\hline & & & & $\mathrm{P}_{2} \mathrm{O}_{5}$ & $\begin{array}{l}\text { Acid } \\
\text { insoluble }\end{array}$ & & \\
\hline $\begin{array}{l}P-8 \\
P-7 \\
P-6 \\
P-5 \\
P-4\end{array}$ & $\begin{array}{l}\text { Chert, argillaceous } \\
\text { Mudstone, cherty } \\
\text { Mudstone, cherty } \\
\text { Chert, argillaceous } \\
\text { Mudstone }\end{array}$ & $\begin{array}{l}-- \\
-- \\
-- \\
--\end{array}$ & $\begin{array}{l}3.5 \\
3.4 \\
1.7 \\
2.9 \\
1.5\end{array}$ & $\begin{array}{l}-- \\
-- \\
-- \\
--\end{array}$ & $\begin{array}{l}-- \\
-- \\
-- \\
--\end{array}$ & $\begin{array}{l}171.8 \\
175.2 \\
176.9 \\
179.8 \\
181.3\end{array}$ & $\begin{array}{l}-- \\
-- \\
-- \\
--\end{array}$ \\
\hline $\begin{array}{l}P-3 \\
P-2 \\
P-1\end{array}$ & 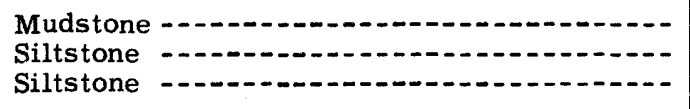 & $\begin{array}{c}6591-\text { RAS } \\
-- \\
--\end{array}$ & $\begin{array}{r}.7 \\
1.4 \\
3.3\end{array}$ & $\begin{array}{l}7.3 \\
-- \\
--\end{array}$ & $\begin{array}{c}68.1 \\
-- \\
--\end{array}$ & $\begin{array}{l}182.0 \\
183.4 \\
186.7\end{array}$ & $\begin{array}{l}-- \\
-- \\
--\end{array}$ \\
\hline
\end{tabular}

Lower member of Park City formation (?)

\begin{tabular}{|c|c|}
\hline $\begin{array}{l}L-62 \\
\mathrm{~L}-61 \\
\mathrm{~L}-60 \\
\mathrm{~L}-59 \\
\mathrm{~L}-58\end{array}$ & $\begin{array}{l}\text { Covered interval } \\
\text { Sandstone } \\
\text { Sandstone } \\
\text { Sandstone } \\
\text { Sandstone }\end{array}$ \\
\hline $\begin{array}{l}L-57 \\
L-56 \\
L-55 \\
L-54 \\
L-53\end{array}$ & $\begin{array}{l}\text { Covered interval } \\
\text { Sandstone, carbonatic } \\
\text { Covered interval } \\
\text { Sandstone, carbonatic } \\
\text { Sandstone, carbonatic }\end{array}$ \\
\hline $\begin{array}{l}L-52 \\
L-51 \\
L-50 \\
L-49 \\
L-48\end{array}$ & $\begin{array}{l}\text { Covered interval } \\
\text { Covered interval } \\
\text { Carbonate rock, cherty } \\
\text { Covered interval } \\
\text { Carbonate rock }\end{array}$ \\
\hline $\begin{array}{l}L-47 \\
\mathrm{~L}-46 \\
\mathrm{~L}-45 \\
\mathrm{~L}-44 \\
\mathrm{~L}-43\end{array}$ & $\begin{array}{l}\text { Siltstone } \\
\text { Carbonate rock, cherty } \\
\text { Covered interval } \\
\text { Covered interval } \\
\text { Covered interval }\end{array}$ \\
\hline $\begin{array}{l}L-42 \\
L-41 \\
L-40 \\
L-39 \\
L-38\end{array}$ & $\begin{array}{l}\text { Carbonate rock } \\
\text { Sandstone, carbonatic } \\
\text { Covered interval } \\
\text { Carbonate rock, cherty } \\
\text { Carbonate rock }\end{array}$ \\
\hline $\begin{array}{l}L-37 \\
L-36\end{array}$ & $\begin{array}{l}\text { Siltstone, carbonatic } \\
\text { Chert and carbonatic siltstone }\end{array}$ \\
\hline
\end{tabular}

\begin{tabular}{l|r}
-- & 1.8 \\
-- & 1.1 \\
-- & 5.7 \\
-- & 5.1 \\
& 8.0 \\
-- & 22.0 \\
-- & 40.0 \\
-- & 13.0 \\
-- & 8.0 \\
-- & 19.5 \\
& 11.0 \\
-- & 50.0 \\
-- & 13.0 \\
-- & 15.0 \\
-- & 3.8 \\
-- & 1.1 \\
& 3.0 \\
-- & 10.0 \\
-- & 10.0 \\
-- & 9.0 \\
-- & 15.0 \\
- & 16.0 \\
-- & 3.0 \\
-- & 1.3 \\
-- & 5.4 \\
-- & 4.0 \\
- & 2.4 \\
-- &
\end{tabular}

\begin{tabular}{l|l|}
-- & -- \\
-- & -- \\
-- & - \\
-- & -- \\
\hline- & -- \\
-- & -- \\
-- & - \\
-- & -- \\
-- & - \\
-- & -- \\
-- & -- \\
-- & -- \\
-- & -- \\
-- & -- \\
-- & -- \\
-- & -- \\
-- & -- \\
-- & -- \\
-- & -- \\
-- & -- \\
-- & -- \\
-- & -- \\
-- & -- \\
-- & -- \\
-- & -- \\
-- & -- \\
&
\end{tabular}

\begin{tabular}{r|r}
1.8 & -- \\
2.9 & -- \\
8.6 & -- \\
13.7 & -- \\
21.7 & - \\
43.7 & -- \\
83.7 & -- \\
96.7 & -- \\
104.7 & -- \\
124.2 & -- \\
135.2 & \\
185.2 & -- \\
198.2 & -- \\
213.2 & -- \\
217.0 & -- \\
218.1 & -- \\
221.1 & \\
231.1 & -- \\
241.1 & -- \\
250.1 & -- \\
265.1 & -- \\
281.1 & -- \\
284.1 & \\
285.4 & -- \\
290.8 & -- \\
294.8 & - \\
297.2 & -- \\
& - \\
& \\
& -- \\
& \\
21 &
\end{tabular}




\begin{tabular}{|c|c|c|c|c|c|c|c|}
\hline $\begin{array}{l}L-35 \\
L-34 \\
L-33 \\
L-32 \\
L-31\end{array}$ & $\begin{array}{l}\text { Covered interval } \\
\text { Siltstone, carbonatic } \\
\text { Carbonate rock, sandy } \\
\text { Siltstone } \\
\text { Mudstone, carbonatic }\end{array}$ & $\begin{array}{l}-- \\
-\infty \\
-- \\
-- \\
--\end{array}$ & $\begin{array}{r}12.0 \\
1.5 \\
1.7 \\
3.4 \\
1.7\end{array}$ & $\begin{array}{l}-- \\
-- \\
-- \\
--\end{array}$ & $\begin{array}{l}-- \\
-- \\
-- \\
--\end{array}$ & $\begin{array}{l}309.2 \\
310.7 \\
312.4 \\
315.8 \\
317.5\end{array}$ & $\begin{array}{l}-- \\
-- \\
-- \\
--\end{array}$ \\
\hline $\begin{array}{l}L-30 \\
L-29 \\
L-28 \\
L-27 \\
L-26\end{array}$ & $\begin{array}{l}\text { Siltstone } \\
\text { Siltstone } \\
\text { Siltstone } \\
\text { Covered interval } \\
\text { Siltstone }-0.00\end{array}$ & $\begin{array}{l}-- \\
-- \\
-- \\
-- \\
--\end{array}$ & $\begin{array}{r}2.2 \\
1.9 \\
1.3 \\
7.0 \\
.9\end{array}$ & $\begin{array}{l}-- \\
-- \\
-- \\
--\end{array}$ & $\begin{array}{l}-- \\
-- \\
-- \\
--\end{array}$ & $\begin{array}{l}319.7 \\
321.6 \\
322.9 \\
329.9 \\
330.8\end{array}$ & $\begin{array}{l}-- \\
-- \\
-- \\
--\end{array}$ \\
\hline $\begin{array}{l}L-25 \\
L-24 \\
L-23 \\
L-22 \\
L-21\end{array}$ & $\begin{array}{l}\text { Siltstone } \\
\text { Silts tone } \\
\text { Silts tone } \\
\text { Mudstone } \\
\text { Silts tone }-2.00\end{array}$ & $\begin{array}{l}-- \\
-- \\
-- \\
--\end{array}$ & $\begin{array}{r}1.7 \\
1.2 \\
4.2 \\
1.8 \\
.9\end{array}$ & $\begin{array}{l}-- \\
-- \\
-- \\
--\end{array}$ & $\begin{array}{l}-- \\
-- \\
-- \\
--\end{array}$ & $\begin{array}{l}332.5 \\
333.7 \\
337.9 \\
339.7 \\
340.6\end{array}$ & $\begin{array}{l}-- \\
-- \\
--\end{array}$ \\
\hline $\begin{array}{l}L-20 \\
L-19 \\
L-18 \\
L-17 \\
L-16\end{array}$ & $\begin{array}{l}\text { Carbonate rock, sandy } \\
\text { Carbonate rock, sandy } \\
\text { Siltstone, carbonatic } \\
\text { Sandstone, carbonatic } \\
\text { Siltstone, carbonatic }\end{array}$ & $\begin{array}{l}-- \\
-- \\
-- \\
-- \\
--\end{array}$ & $\begin{array}{r}2.2 \\
4.7 \\
.8 \\
1.7 \\
2.9\end{array}$ & $\begin{array}{l}-- \\
-- \\
-- \\
-- \\
--\end{array}$ & $\begin{array}{l}-- \\
-- \\
-- \\
--\end{array}$ & $\begin{array}{l}342.8 \\
347.5 \\
348.3 \\
350.0 \\
352.9\end{array}$ & $\begin{array}{l}-- \\
-- \\
-- \\
--\end{array}$ \\
\hline $\begin{array}{l}L-15 \\
L-14 \\
L-13 \\
L-12 \\
L-11\end{array}$ & $\begin{array}{l}\text { Carbonate rock, sandy } \\
\text { Carbonate rock, sandy } \\
\text { Carbonate rock } \\
\text { Carbonate rock, sandy } \\
\text { Sandstone }\end{array}$ & $\begin{array}{l}-- \\
-- \\
-- \\
-- \\
--\end{array}$ & $\begin{array}{r}1.1 \\
3.3 \\
18.0 \\
13.0 \\
6.0\end{array}$ & $\begin{array}{l}-- \\
-- \\
-- \\
--\end{array}$ & $\begin{array}{l}-- \\
-- \\
-- \\
--\end{array}$ & $\begin{array}{l}354.0 \\
357.3 \\
375.3 \\
388.3 \\
394.3\end{array}$ & $\begin{array}{l}-- \\
-- \\
-- \\
--\end{array}$ \\
\hline $\begin{array}{l}L-10 \\
L-9 \\
L-8 \\
L-7 \\
L-6\end{array}$ & $\begin{array}{l}\text { Carbonate rock } \\
\text { Covered interval } \\
\text { Carbonate rock } \\
\text { Carbonate rock } \\
\text { Covered interval }\end{array}$ & $\begin{array}{l}-- \\
-- \\
-- \\
-- \\
--\end{array}$ & $\begin{array}{l}20.0 \\
70.0 \\
29.0 \\
50.0 \\
12.0\end{array}$ & $\begin{array}{l}-- \\
-- \\
-- \\
--\end{array}$ & $\begin{array}{l}-- \\
-- \\
-- \\
-- \\
--\end{array}$ & $\begin{array}{l}414.3 \\
484.3 \\
513.3 \\
563.3 \\
575.3\end{array}$ & $\begin{array}{l}-- \\
-- \\
-- \\
--\end{array}$ \\
\hline $\begin{array}{l}L-5 \\
L-4 \\
L-3 \\
L-2 \\
L-1\end{array}$ & $\begin{array}{l}\text { Carbonate rock, sandy } \\
\text { Carbonate rock } \\
\text { Covered interval } \\
\text { Carbonate rock } \\
\text { Carbonate rock }\end{array}$ & $\begin{array}{l}-- \\
-- \\
-- \\
-- \\
--\end{array}$ & $\begin{array}{l}16.0 \\
22.0 \\
18.0 \\
32.0 \\
20.0\end{array}$ & $\begin{array}{l}-- \\
-- \\
-- \\
--\end{array}$ & $\begin{array}{l}-- \\
-- \\
-- \\
-- \\
--\end{array}$ & $\begin{array}{l}591.3 \\
613.3 \\
631.3 \\
663.3 \\
683.3\end{array}$ & $\begin{array}{l}-- \\
-- \\
-- \\
--\end{array}$ \\
\hline \multicolumn{8}{|c|}{ Diamond Creek sandstone (?) } \\
\hline$D-1$ & Sandstone, calcareous - & -- & (?) & -- & -- & -- & -- \\
\hline
\end{tabular}




\section{Little Diamond Creek, Utah, lot 1376}

Lower part of phosphatic shale member of Park City formation measured and sampled in old open pit mine under lease to American Smelting and Refining

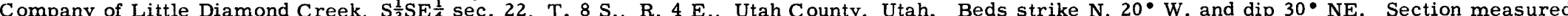
by $T$. M. Cheney and sampled by $R$. G. Waring in October 1951. Samples analyzed for $P_{2} O_{-}$and acid insoluble by U. S. Bureau of Mines laboratory Albany, Oreg., and for other constituents by Trace Elements Section laboratory, U. S. Geological Survey, Washington, D. C.

\begin{tabular}{|c|c|c|c|c|c|c|c|c|c|c|}
\hline \multirow{2}{*}{$\begin{array}{r}\text { Bed } \\
\text { no. }\end{array}$} & \multirow[b]{2}{*}{ Rock description } & \multirow{2}{*}{$\begin{array}{c}\text { Sample } \\
\text { no. }\end{array}$} & \multirow{2}{*}{$\begin{array}{c}\text { Thickness } \\
\text { (feet) }\end{array}$} & \multicolumn{5}{|c|}{ Chemical analyses (percent) } & \multirow{2}{*}{$\begin{array}{l}\text { Cumulative } \\
\text { thickness } \\
\text { (feet) }\end{array}$} & \multirow{2}{*}{$\begin{array}{l}\text { Thickness } x \\
\text { percent } \mathrm{P}_{2} \mathrm{O}_{5} \\
\text { (cumulative) }\end{array}$} \\
\hline & & & & $\mathrm{P}_{2} \mathrm{O}_{5}$ & $\mathrm{Al}_{2} \mathrm{O}_{3}$ & $\mathrm{Fe}_{2} \mathrm{O}_{3}$ & $\begin{array}{l}\text { Loss on } \\
\text { ignition }\end{array}$ & $\begin{array}{c}\text { Acid } \\
\text { insoluble }\end{array}$ & & \\
\hline
\end{tabular}

Phosphatic shale member of Park City formation-basal part only

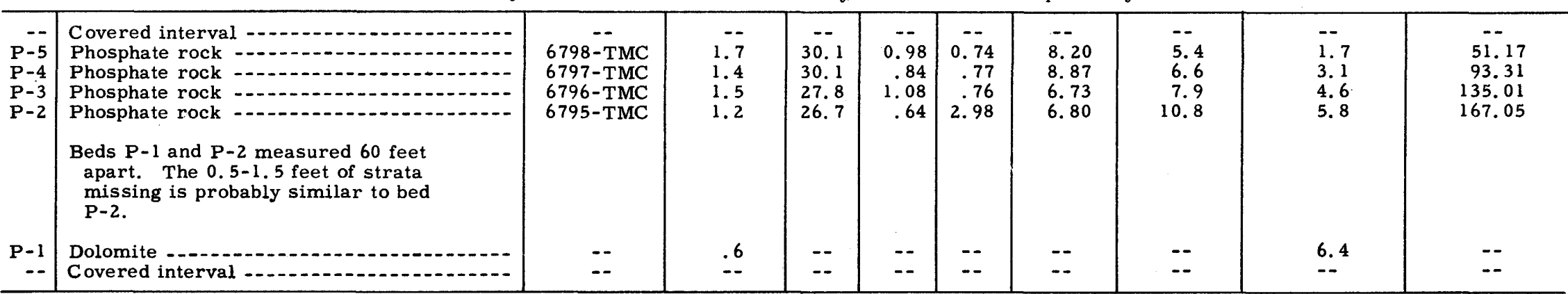

